\title{
Single Photon Emission Computed Tomography (SPECT) Myocardial Perfusion Imaging Guidelines: Instrumentation, Acquisition, Processing, and Interpretation
}

Sharmila Dorbala, MD, MPH, ${ }^{\text {a }}$ Karthik Ananthasubramaniam, MD, ${ }^{b}$ Ian S. Armstrong, PhD, MIPEM, ${ }^{\mathrm{C}}$ Panithaya Chareonthaitawee, $\mathrm{MD},{ }^{\mathrm{d}}$ E. Gordon DePuey, MD, ${ }^{\mathrm{e}}$ Andrew J. Einstein, $\mathrm{MD}, \mathrm{PhD},{ }^{\mathrm{f}}$ Robert J. Gropler, $\mathrm{MD},{ }^{\mathrm{g}}$ Thomas A. Holly, MD, ${ }^{\mathrm{h}}$ John J. Mahmarian, MD, ${ }^{\mathrm{i}}$ Mi-Ae Park, PhD,

Donna M. Polk, MD, MPH, ${ }^{\text {a }}$ Raymond Russell III, MD, PhD, ${ }^{k}$ Piotr J. Slomka, PhD, ${ }^{1}$ Randall C. Thompson, MD, ${ }^{\mathrm{m}}$ and R. Glenn Wells, $\mathrm{PhD}^{\mathrm{n}}$

a Brigham and Women's Hospital, Harvard Medical School, Boston, MA

b Henry Ford Hospital, Detroit, MI

c Manchester University Foundation Trust, Manchester, UK

d Mayo Clinic, Rochester, MN

e Mount Sinai, New York, NY

f Columbia University Medical Center and New York-Presbyterian Hospital, New York, NY

$\mathrm{g}$ Washington University, St. Louis, MO

h Northwestern University Feinberg School of Medicine, Chicago, IL

i Houston Methodist Hospital and Weill Cornell Medical College, Houston, TX

j Harvard Medical School, Boston, MA

k Rhode Island Hospital, Providence, RI

I Cedars-Sinai, Los Angeles, CA

m St. Luke's Mid America Heart Institute, Kansas City, MO

n University of Ottawa Heart Institute, Ottawa, Canada

doi: $10.1007 / \mathrm{s} 12350-018-1283-y$

\begin{tabular}{|llll|}
\hline Abbreviations & MPI & \multicolumn{2}{l|}{ Myocardial perfusion imaging/images } \\
AC & Attenuation correction & RV & Right ventricular \\
CZT & Cadmium zinc telluride & SPECT & Single photon emission computed \\
ECG & Electrocardiographic & & tomography \\
LV & Left ventricular & ${ }^{99 \mathrm{~m}} \mathrm{Tc}$ & ${ }^{99 \mathrm{~m}}$ Technetium \\
EF & Ejection fraction & & \\
FDG & ${ }^{18}$ F-fluorodeoxyglucose & & \\
\hline
\end{tabular}

Karthik Ananthasubramaniam, E. Gordon DePuey, John J. Mahmarian, Raymond Russell III, Randall C. Thompson, and R. Glenn Wells are section lead authors.

This document was approved for publication by the governing body of ASNC and was endorsed by the Society of Nuclear Medicine and Molecular Imaging (SNMMI).
Reprint requests: Sharmila Dorbala, MD, MPH, Brigham and Women's Hospital, Harvard Medical School, Boston, MA; sdorbala@bwh.harvard.edu

J Nucl Cardiol 2018;25:1784-1846.

$1071-3581 / \$ 34.00$

Copyright $@ 2018$ American Society of Nuclear Cardiology. 


\section{Table of Contents}

\section{INTRODUCTION}

SPECT INSTRUMENTATION QUALITY ASSURANCE AND PERFORMANCE

Detectors

Scintillation crystal (single non-pixelated crystal) with PMT array

Scintillation crystals (pixelated) with PMT or solid-state photodiode array

Semiconductor radiation detectors

Energy Resolution

Spatial Resolution

Detector Sensitivity

Count-Rate Limitations

Collimators

Parallel-hole collimation

Converging-hole collimation

Pinhole collimation

System Design

Conventional SPECT

Dedicated cardiac SPECT

Upright CZT camera with sweeping parallel-hole collimators (D-SPECT, Spectrum Dynamics, Haifa, Israel)

CZT with multiple single-pinhole detectors (Discovery NM530c, General Electric, Waukesha, WI)

Indirect photon detection with solid-state photodiodes (Cardius, Digirad, Suwanee, GA)

Conventional cameras with cardio-focal collimators (IQSPECT, Siemens Healthineers, Malvern, PA)

Other designs

Data sampling

Patient configuration

SPECT systems with photon sources for attenuation correction

SPECT systems with CT for attenuation correction

QC Procedures

Camera acceptance

SPECT QC procedures

Energy calibration check

Uniformity test

High-count flood-field uniformity corrections

Resolution and linearity test

Sensitivity test

Center-of-rotation calibration

Multipurpose plexiglass phantoms

Radionuclide TCT QC procedures

Energy Calibration Check

Transmission source mechanics

Source strength

CT QC procedures

CT number calibration

Field uniformity

Combined TCT and SPECT QC procedures

Registration

Attenuation correction accuracy

Recommendations for SPECT Instrumentation, Quality Assurance, and Performance

\section{ACQUISITION}

Activity

Position

Delay Time for Imaging

Energy Windows

Collimator
Angular Sampling Range

Number of Projections

Orbit Type

Pixel and Matrix Size

Acquisition Type

Acquisition Time

Gating

Attenuation Correction

Recommendations for SPECT MPI Acquisition

PROCESSING

Filtering

Reconstruction

Filtered backprojection

Iterative reconstruction

Reduced count density reconstructive techniques

Resolution recovery

Noise compensation/reduction

Pre-determined SPECT acquisition times

Attenuation Correction

Attenuation

Optimization of CT for AC

Practical considerations

Axial coverage of CT

Image checks prior to $\mathrm{CT}$ scan

Pitfalls

Truncation

Misregistration

Solid-State Cameras

Reconstruction

List mode and motion correction

Artifact mitigation with new scanners

Reorientation

Cine Review

Recommendations for SPECT MPI Processing

INTERPRETATION

General Comments

Display

Recommended medium for display

Conventional slice display of SPECT images

Three-dimensional display

Evaluation of the Images for Technical Sources of Error

Patient motion

Attenuation artifacts and attenuation correction

Reconstruction artifacts

Myocardial count statistics

Initial Image Analysis and Interpretation

Ventricular dilation

Lung uptake

Right ventricular uptake

Noncardiac physiological and pathological findings

Perfusion defect location

Perfusion defect severity and extent

Qualitative

Semiquantitative

Quantitative

Reversibility

Gated Myocardial Perfusion SPECT

Gated SPECT display

Gated SPECT QC

Gated SPECT: regional wall motion and thickening

Left ventricular ejection fraction and volumes

Integration of perfusion and function results

LV systolic dyssynchrony

Clinical Interpretation of AC SPECT Studies 
Final Interpretation of MPI with Clinical and Stress-Test Data

Myocardial Viability

Qualitative assessment

Semiquantitative assessment

Quantitative assessment

Clinical utility of viability imaging

Recommendations for Interpretation of SPECT MPI

SPECT MYOCARDIAL BLOOD FLOW QUANTITATION

Introduction

Acquisition

Processing

STRESS-FIRST/STRESS-ONLY IMAGING

Utility of Stress-First / Stress-Only Imaging

Limitations of Stress-First / Stress-Only SPECT Imaging

Patient Selection for a Stress-First / Stress-Only Imaging Protocol

Criteria for a Normal Stress-First SPECT

Recommendations for the Use of a Stress-First SPECT

PATIENT CENTERED MPI

Introduction

Selection of Imaging Protocol/Scanner

Minimizing Radiation Exposure

Matching the Clinical Question to the Imaging and Stress Testing Protocol

Combining CT Calcium Score with MPI

Recommendations for Patient-Centered MPI

Appendix

References

\section{INTRODUCTION}

The purpose of this document is to update the "ASNC Imaging Guidelines for Nuclear Cardiology Procedures: Single photon-emission computed tomography" ${ }^{\prime 1}$ in several areas in which novel evidence has emerged since its publication. Recent advances in Single Photon Emission Computed Tomography (SPECT) have fundamentally changed acquisition, processing, and interpretation of myocardial perfusion images using new technology. Myocardial perfusion imaging (MPI) can now be personalized and tailored to the individual patient and the clinical question. This publication is designed to provide MPI guidelines for conventional and novel SPECT for qualified medical professionals engaged in the practice of nuclear cardiology. While the information supplied in this document has been carefully reviewed by experts in the field, the document should not be considered as medical advice or a professional service. We recognize that SPECT technology is evolving rapidly and that these recommendations may need further revision in the near future. Hence, the imaging guidelines described in this publication should be applied clinically only after review and approval by qualified local institutional physicians and technologists.

The current update includes the latest knowledge on SPECT MPI incorporated into existing and new sections of the document. The instrumentation quality assurance and performance section was updated with advances in novel detectors, novel cameras, and hybrid SPECT/computed tomography (CT) equipment. Acquisition protocols for MPI on existing cameras were updated and new protocols added for MPI with semiconductor detector cameras. The processing section now has expanded coverage of iterative reconstruction and attenuation correction (AC). New information was added on reduced count density reconstruction techniques and reconstruction for solid state cameras. The interpretation section now includes guidance on interpretation of MPI from dedicated cardiac cameras. The section on reporting of SPECT MPI has been removed and is now included in the new American Society of Nuclear Cardiology (ASNC) reporting guideline document. ${ }^{2}$ Finally, several figures and new sections on myocardial blood flow with SPECT, stressfirst/stress-only imaging, and patient centered MPI are added to this update.

This document was approved for publication by the governing body of ASNC and was endorsed by the Society of Nuclear Medicine and Molecular Imaging (SNMMI).

\section{SPECT INSTRUMENTATION QUALITY ASSURANCE AND PERFORMANCE}

The proper choice of equipment to acquire clinical data and a well-designed quality assurance (QA) program are both essential requirements for optimizing diagnostic accuracy and ensuring consistent, high-quality MPI. The following guidelines are intended to provide an appropriate means of assessing SPECT equipment function for nuclear cardiology imaging. Because the optimal manner to perform specific tests varies considerably between different types of imaging equipment, this document is not intended to replace manufacturers' recommendations.

For decades, the design of SPECT cameras has remained essentially unchanged, consisting of one or more large-area scintillation or Anger cameras comprised of a single $\mathrm{NaI}(\mathrm{Tl})$ crystal with an array of large photomultiplier tubes (PMTs) and a parallel-hole collimator. The QA and performance of these cameras are well understood, and detailed guidelines are presented in this document. In recent years, however, novel and dedicated cardiac SPECT cameras have emerged with significantly different detector, collimator, and system designs. ${ }^{3}$ Because this technology is rapidly evolving, detailed guidelines for QA testing do not yet exist for these new designs. Until detailed guidelines become available, the user is responsible for assessing and following vendor recommendations, and must devise acceptable QA standards specific to the camera design. To assist in this endeavor, the basic design concepts and the implications on performance and QA of conventional and novel dedicated cardiac SPECT cameras are summarized in this document.

\section{Detectors}

Detectors are the key component of a SPECT system and are responsible for collecting the photon 
events, estimating the photon energy and position of detection, and thus generating data for subsequent image reconstruction. The ability to perform these tasks depends on the design, materials, and electronics of the detectors. Energy resolution, sensitivity, and spatial resolution are the primary variables that dictate the performance of a SPECT detector.

Scintillation crystal (single non-pixelated crystal) with PMT array. The majority of SPECT systems are based on Anger camera technology where one or more detectors rotate around the body of the patient. Anger cameras consist of a single scintillation crystal (Figure 1A), which absorbs incident gamma rays and scintillates or emits light in response. Behind the crystal is a bank of PMTs and electronics to compute gamma-ray energy and the location of scintillation within the crystal. Anger camera $\mathrm{NaI}(\mathrm{Tl})$ crystals are typically $9.5 \mathrm{~mm}(3 / 8$ inch) thick, although they can range from $6.35 \mathrm{~mm}$ to 15.9 $\mathrm{mm}$ (1/4 inch to $5 / 8$ inches). The thicker the crystal, the greater the sensitivity of the detector, because of the increased probability that a gamma ray passing through the crystal will interact. However, with thicker crystals, the spread of the emitted light photons produced from the scintillation is greater and the precision of the calculated location of the gamma-ray interaction is less, resulting in a poorer intrinsic resolution of the camera. Thicker crystals provide increased sensitivity for higher energy gamma rays, but $9.5 \mathrm{~mm}(3 / 8$-inch) crystals are preferred for ${ }^{99 \mathrm{~m}}$ Technetium $\left({ }^{99 \mathrm{~m}} \mathrm{Tc}\right)$ and ${ }^{201}$ thallium $\left({ }^{201} \mathrm{Tl}\right)$ tracers as they already stop approximately $90 \%$ of $140 \mathrm{keV}$ photons.

Scintillation crystals (pixelated) with PMT or solid-state photodiode array. An array of scintillation crystals is an alternative to the single-crystal Anger detector design. A large number of small scintillation crystals (e.g., $6 \mathrm{~mm} \mathrm{CsI}[\mathrm{Tl}]$ cubes) are coated with reflective material and packed into an array (Figure 1B). An advantage of the pixelated design is that the scintillation light is much more focused than a single-crystal design and can be converted into an electrical signal by a solid-state photodiode array instead of conventional PMTs, thereby making the detector much more compact. ${ }^{4,5}$ Pixelated detectors are capable of very high counting rates due to their isolated light pulses and have been used in first-pass cardiac scintigraphy.

Semiconductor radiation detectors. Recently, alternative SPECT systems have been introduced that are based on small semiconductor detector modules which directly detect the gamma rays (Figure 1C), without the use of scintillation crystal. In these solid-state detectors, gamma rays are absorbed into the semiconductor material, which directly generate electron-hole pairs that are pulled to the end plates through an applied electric field. The collected charge from the electron-hole pairs is used to determine the location and energy of the gamma ray. Currently, these detectors are made of cadmium zinc telluride (CZT). The direct conversion generates a larger signal than the NaI-PMT (Anger) approach leading to improved energy resolution. ${ }^{6,7}$ The small size of the detector modules has made a number of innovative camera designs possible (section “System Design”).

\section{Energy Resolution}

Photons emitted by the radionuclide, which scatter before reaching the detector, have reduced energy compared to those that reach the detector without intervening interactions. Including the scattered photons in the projection data reduces image contrast, thereby reducing the ability to discriminate areas of hypoperfusion from those of neighboring normal perfusion. The fraction of scattered photons that can be excluded from the acquired data based on their lower energy is dependent on the energy resolution of the camera.

A symmetric energy window of $15 \%$ to $20 \%$ centered around the $140 \mathrm{keV}$ energy peak of the emitted photons is standard for ${ }^{99 \mathrm{~m}} \mathrm{Tc}$. This window width provides an appropriate tradeoff between accepting unscattered-photons and rejecting the scattered-photons. The lower energy and greater width of the ${ }^{201} \mathrm{Tl} 70 \mathrm{keV}$ photopeak require a wider energy window, and an energy window setting of $30 \%$ is appropriate for the $70 \mathrm{keV}$ peak of ${ }^{201} \mathrm{Tl}$. A $15 \%$ to $20 \%$ energy window is appropriate for the 167 $\mathrm{keV}$ peak. The absolute energy calibration can be unreliable at the low energy of ${ }^{201} \mathrm{Tl}$, falling at slightly different energy positions in the spectrum of different cameras. Thus, energy peak and window settings should be established for each individual camera based on the energy spectrum display. ${ }^{8}$ For most modern detectors with scintillation crystals, the energy resolution is expected to be $9 \%$ to $10 \%$ for the $140-\mathrm{keV}$ photons from ${ }^{99 \mathrm{~m}} \mathrm{Tc}$, and $15 \%$ to $17 \%$ for the $72-\mathrm{keV}$ photons from ${ }^{201} \mathrm{Tl} .{ }^{9}$ For CZT detectors, an energy resolution of $5 \%$ to $6 \%$ has been reported for ${ }^{99 \mathrm{~m}} \mathrm{Tc} .{ }^{6,7}$

Modern gamma cameras allow simultaneous acquisition of data in multiple energy windows. Data from additional lower energy windows can be used to correct for the remaining scatter detected in the primary energy window (Section "Processing").

\section{Spatial Resolution}

Spatial resolution quantifies the size of the smallest object that can be resolved reliably, and is often expressed as the full width at half maximum (FWHM) of a point spread function. The total resolution of the acquired projection data depends on the intrinsic resolution 

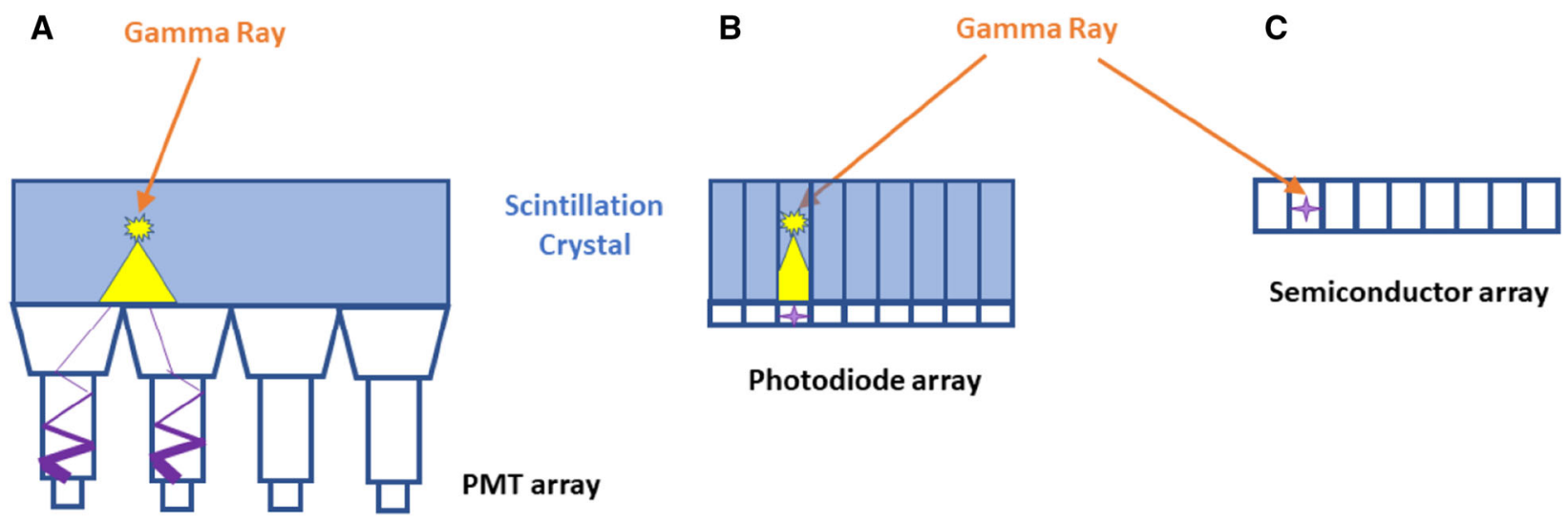

Figure 1. SPECT Detectors. (A) A monolithic scintillation crystal backed by a photomultiplier (PMT) array. The gamma ray (orange) is converted into a shower of light photons (yellow), which are converted into an electronic signal (purple). (B) Pixelated scintillation crystals backed by a photodiode array. The light from the gamma ray interaction is funneled to a solid-state photodiode array, which converts the light into an electronic signal. (C) A pixelated semiconductor detector: The gamma ray is converted directly into an electronic signal.

Table 1. Characteristics of detectors for ${ }^{99 \mathrm{~m}} \mathrm{Tc}(140-\mathrm{keV}$ photons)

\begin{tabular}{lccccccc}
\hline & $\begin{array}{c}\text { Position/ } \\
\text { Energy } \\
\text { determi } \\
\text {-nation }\end{array}$ & $\begin{array}{c}\text { Thick } \\
\text {-ness } \\
\text { (mm) }\end{array}$ & $\begin{array}{c}\text { Energy } \\
\text { Resolution } \\
\text { (\%) }\end{array}$ & $\begin{array}{c}\text { Detector } \\
\text { Pixel Size } \\
\text { (mm) }\end{array}$ & $\begin{array}{c}\text { Intrinsic } \\
\text { Spatial } \\
\text { Resolution } \\
\text { (mm) }\end{array}$ & $\begin{array}{c}\text { Intrinsic } \\
\text { Efficiency } \\
\text { (\%)* }\end{array}$ & $\begin{array}{c}\text { Count Rate } \\
\text { (20\% loss) } \\
\text { (kcps) }\end{array}$ \\
\hline Nal & PMT & 9.5 & 10 & variable & 4 & 90 & $>250$ \\
CSI & PD & 6 & 10 & 6 & 6 & 90 & $>5000$ \\
CZT & N/A & 5 & 6 & 2.5 & 2.5 & 85 & $>600$ \\
\hline
\end{tabular}

* Does not include the effect of the photopeak window. Calculated from the National Institute of Standards and Technology attenuation coefficient tables

CsI, Cesium iodide (TI doped); CZT, cadmium zinc telluride; $k c p s, 10^{3}$ counts per second; $m m$, millimeter; $P D$, photodiode; $P M T$, photomultiplier tube; Nal, sodium iodide (thallium doped)

(resolution measured with no collimator and with the source on the face of the detector) and the collimator resolution (section "Collimators"). The intrinsic resolution refers to the precision of determining the point of interaction of the gamma ray with the detector. With Anger cameras, the intrinsic resolution is dependent on the uncertainties in the PMT signal strengths and the electronic positioning logic circuitry. The Anger camera intrinsic resolution is typically $3.5 \mathrm{~mm}$ to $4.0 \mathrm{~mm}$ for ${ }^{99 \mathrm{~m}} \mathrm{Tc}$ (Table 1). For pixelated solid-state detectors, the intrinsic resolution is primarily a function of the detector element size.

\section{Detector Sensitivity}

The image quality of gamma-camera images is largely determined by the signal-to-noise ratio. The greater the sensitivity of the device, the more counts are available, and the higher the signal-to-noise ratio. Thus, it is desirable to have the most sensitive device available to form the clinical images. The sensitivity of the camera is driven by three main factors: 1) the number of detectors (i.e., the solid angle coverage of the system design), 2) the collimators used with each detector, and 3) the intrinsic efficiency of the detector (detector sensitivity). The intrinsic efficiency of all clinical detectors is very high (Table 1), and actually higher for standard cameras then for CZT cameras, so the overall system sensitivity is primarily driven by collimator choice (section "Collimators") and system design (section “'System Design”).

\section{Count-Rate Limitations}

At very high photon detection rates, the camera electronics can have difficulty analyzing every photon. The missed counts, often referred to as dead-time effects, can affect the estimated distribution of the 
A

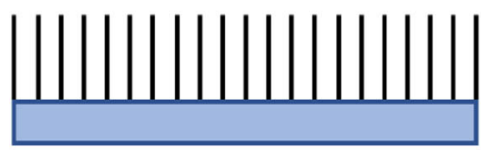

Parallel Hole
B

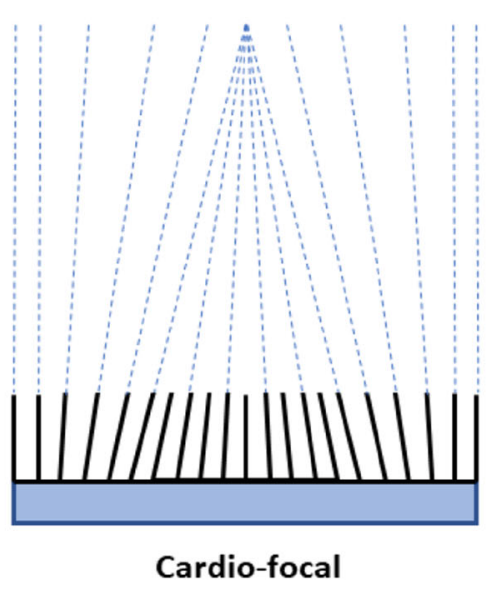

C

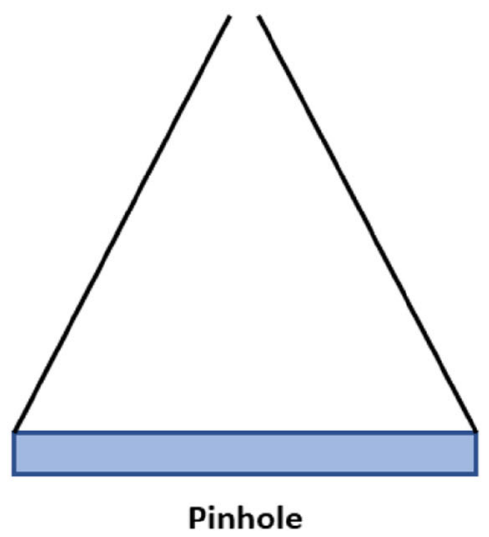

Figure 2. There are three types of collimator in commercial use for cardiac SPECT: (A) the parallel-hole collimator, (B) the cardio-focal collimator, and (C) the pinhole collimator. Transverse views through the collimator (black lines) are shown; the collimators have similar arrangement in the axial direction. The location of the detector is shown as a light-blue box. The septa for the cardio-focal collimator have been extended as dashed lines to better show their relative orientation.

Table 2. Typical performance parameters for low-energy $(<150 \mathrm{keV})$ parallel-hole collimators

\section{Collimator type}

Ultra-high resolution

High resolution

All/general purpose

High sensitivity
Resolution (FWHM at $10 \mathrm{~cm}$ )

$6.0 \mathrm{~mm}$

$7.8 \mathrm{~mm}$

$9.3 \mathrm{~mm}$

$13.2 \mathrm{~mm}$

Efficiency (\%)

Relative efficiency

* A relative efficiency of 1 corresponds approximately to a collimator efficiency of $0.017 \%$, whereas efficiency is defined as the fraction of gamma rays and X-rays passing through the collimator per gamma ray and X-ray emitted by the source $F W H M$, full width at half maximum; $\mathrm{cm}$, centimeters; $\mathrm{mm}$, millimeters

radiotracer in the body. A count-rate related performance metric of SPECT detectors is count rate at which dead-time effects result in the loss of $20 \%$ of the expected count rate (Table 1). One future clinical application where dead-time effects may need to be considered is dynamic imaging of myocardial blood flow (MBF). During the initial time frames, the entire injected bolus of tracer may be in the field of view (FOV) at once, leading to very high count rates. Incorrectly accounting for dead-time loses can lead to errors in the quantitative measurement of blood flow. However, for all cardiac myocardial perfusion radiotracers, protocols, and imaging systems currently in clinical use, dead-time effects are not a clinical concern.

\section{Collimators}

Gamma camera imaging requires the incident direction of each detected photon to be known. A collimator defines the photon direction by absorbing photons outside a range of incident angles as specified by the collimator design. By limiting the number of detected photons to a specific direction or small range of directions, the collimator allows the detection of only a very small fraction (typically less than $0.1 \%$ ) of the photons emitted by the patient. However, because a range of incident angles is allowed for the photons passing through the collimator, there is a loss of spatial resolution. Indeed, an inherent tradeoff in the design of SPECT collimators is resolution versus sensitivity. It is possible to improve the spatial resolution of a collimator by further restricting the range of incident angles, but this comes at the expense of reducing sensitivity.

Because of this resolution versus sensitivity tradeoff, the collimator is perhaps the most significant component of the SPECT camera affecting image quality. Most SPECT cameras for nuclear cardiology use conventional parallel-hole collimation. However, it 
has long been recognized that significant improvements in SPECT performance are possible with alternative collimator designs. Coupled with advances in detector and computing technology in recent years, new collimator designs have become practical for nuclear cardiology.

Parallel-hole collimation. The structure of a parallel-hole collimator is similar to a honeycomb, consisting of a large number of narrow channels separated by thin septa (Figure 2A). The range of incident angles passing through the collimator depends on the channel width and length. The septa must be thick enough to absorb photons of the desired energy that are incident at angles greater than the acceptance angle. Parallel-hole collimators are often categorized as low, medium, or high energy depending on the photon energy for which the collimator is designed. Various designs of low-energy collimators are classified as high-resolution, all-purpose, high-sensitivity, etc., depending on the collimator's acceptance angle (the range of accepted incident photon directions). Some typical performance parameters for parallel-hole collimators are given in Table 2.

For gamma cameras, parallel-hole collimation is standard. The low-energy, high-resolution collimator is usually best for ${ }^{99 \mathrm{~m}} \mathrm{Tc}$, although some "all-purpose", collimators can also give excellent results. Imaging with ${ }^{201} \mathrm{Tl}$ is usually best with the low-energy, mediumresolution (all-purpose) collimators because count statistics become limiting when using high-resolution collimators. The difference in medium- and high-resolution collimators is usually that the length of the collimator hole is greater in high-resolution collimators. Medium- and high-resolution collimators have similar near-field resolution, but high-resolution collimators maintain good resolution at a greater distance from the collimator face. The difference is important in SPECT imaging, where the distance from patient to collimator can be greater than in planar imaging. ${ }^{9}$

Collimator selection has substantial influence on the resultant image quality. A confounding aspect of this selection is that collimators with the same name (e.g., 'general purpose') vary in performance from one manufacturer to another. The user should refer to specific imaging protocols for appropriate collimator selection. It is important to perform periodic assessment of collimator integrity, as failure to detect and correct localized reduced sensitivity (e.g. collimator damage) can generate uniformity-related artifacts, including reconstruction artifacts. $^{4,10}$

Converging-hole collimation. With largearea detectors and parallel-hole collimation, only a small fraction of the detector area is used for imaging the myocardium. Converging (fan-beam and cone- beam) collimators allow more of the crystal area to be used in imaging the heart, magnifying the image, and increasing sensitivity. ${ }^{9}$ Converging collimators with a short, fixed focal distance have the potential for truncating portions of the heart and/or chest, especially in large patients. This truncation may generate artifacts.

Another converging collimator design that has reached a commercial stage is the variable-focus conebeam (cardio-focal) collimator (Figure 2B). ${ }^{11,12}$ The center of this collimator focuses on the heart to increase the myocardial counts acquired. For the remainder of the collimator, the focal length increases as one moves toward the edge of the FOV. Data are thus acquired over a FOV similar to a parallel-hole collimator of the same size, but with increased sampling density in the region of the heart and reduced sampling elsewhere. This design allows improved sensitivity for myocardial imaging without introducing truncation artifacts.

Pinhole collimation. A pinhole collimator consists of a small pinhole aperture surrounded by radiationabsorbing material (Figure 2C). Photons must pass through the pinhole to reach the detector. The line connecting the pinhole aperture and the point of detection of the photon specifies the direction of the incident photon. The sensitivity and resolution characteristics of pinhole collimators differ markedly from those of parallel-hole collimators. The sensitivity of a pinhole collimator depends on the inverse square of the distance from the pinhole aperture; it becomes quite large at small distances. It also increases as the square of the pinhole diameter with concurrent loss in spatial resolution. The magnification factor of a pinhole is determined by the ratio of the pinhole-to-detector distance relative to the pinhole-to-source distance. With increased magnification factors, the detector intrinsic resolution has decreased contribution to the total system spatial resolution, which approaches that of the pinhole collimator. A pinhole collimator is most effective for imaging a small object placed close to the pinhole aperture, and is clinically used most often for planar thyroid imaging. A single pinhole collimator tends to have reduced sensitivity compared to a parallel-hole collimator for the same resolution. Using multiple pinholes, however, can greatly increase the sensitivity of the system and thus provide both improved spatial resolution and sensitivity compared to conventional cameras using parallel-hole collimation. Current cardiac systems employ multiple pinholes for tomographic cardiac SPECT imaging, either as multi-pinhole collimators for conventional camera $^{13}$ or as part of a dedicated cardiac camera design (section "System Design').6,14 


\section{System Design}

Conventional SPECT. A common system design for cardiac SPECT is a multipurpose SPECT camera with one or more large-area Anger-camera heads. The camera heads are attached to a gantry, which rotates them around the patient and adjusts their distance relative to the center-of-rotation (COR). Projection images are acquired as the gantry rotates the detectors in a continuous or step-and-shoot mode. An advantage of this SPECT system design is that it is compatible with general nuclear medicine imaging (planar and SPECT) as well as with cardiac SPECT imaging. Cardiac SPECT studies are commonly acquired with a $180^{\circ}$ orbit (right anterior oblique [RAO] to left posterior oblique [LPO] or LPO to RAO). The preferred system configuration is to have two detectors separated by $90^{\circ}$ as they rotate around the heart. The use of two detectors increases system sensitivity and thereby reduces scan time and/or patient radiation exposure. A potential concern with the $90^{\circ}$ configuration is the possibility of truncation of the SPECT projections in the region between the two detectors. Many dual-detector systems also offer a more acute configuration (e.g., $80^{\circ}$ ) and a wider angular coverage (e.g., $100^{\circ}$ ) to avoid possible truncation near the heart.

The various mechanical configurations and detector motions of multipurpose SPECT systems have the potential for misalignment errors and must be included in the laboratory's QA program (e.g., COR correction, multi-detector registration).

Dedicated cardiac SPECT. As nuclear cardiology grew in volume, the market expanded for SPECT cameras designed solely for cardiac imaging. Many cameras emerged with smaller area detectors in a fixed $90^{\circ}$ configuration that had significantly reduced cost, weight, and space requirements. Care in patient positioning, however, is more critical when using these dedicated cardiac SPECT systems, compared to multipurpose SPECT systems with large-area detectors, to avoid truncating the heart and the associated artifacts. Otherwise, QA is similar to the multipurpose SPECT systems.

The growth of dedicated cardiac SPECT systems also led to the development and adoption of novel detector and collimation technologies as described previously. New designs of the gantry, collimators, and detectors have been developed, which allow increased sampling of the myocardial region, and thus allow better local count sensitivity. These systems combine an improvement in spatial resolution and sensitivity. Because of the wide variety of designs and their novelty, their QA requirements may differ from the standard Anger camera with parallel-hole collimation.
Though a detailed QA summary for each design is not presented in this document, areas where the QA or performance may differ are noted. Each laboratory should understand these differences and consult with the vendor's user manuals to develop its own program until such standards and guidelines are developed. Below we describe specific system designs for the dedicated cardiac SPECT systems.

Upright CZT camera with sweeping parallel-hole collimators (D-SPECT, Spectrum Dynamics, Haifa, Israel). In one design, the pixilated CZT detector arrays are mounted in vertical columns and placed in gantry over a $90^{\circ}$ arc around the patient. ${ }^{3,7}$ Each $4 \mathrm{~cm} \times 16 \mathrm{~cm}$ detector is configured with a high-sensitivity parallelhole collimator, which restricts its FOV to a small volume. To cover the entire myocardial region, each detector pivots about its own axis, sweeping its FOV like a searchlight across the entire imaging volume. With a sufficient number of these small pivoting detectors, full-angular sampling of the myocardial region is achieved, allowing reconstruction of highquality images. Available commercial systems employ six to nine detectors. The sweeping FOV of the pivoting detectors provides the flexibility to control the amount of time that is spent acquiring data from each region of the imaging FOV. By spending more time imaging the myocardium and less time imaging the rest of the chest, data collection is more efficient and allows reduced scan time or administered tracer activity. Proprietary iterative reconstruction, which includes resolution recovery and assumptions about heart anatomy, is utilized to compensate for the loss of resolution from high-sensitivity collimation. Patients can be imaged in an upright or semi-supine position on a reclining chair. Attenuation correction is not offered for this system and the vendor recommends 2-position upright/supine imaging to resolve possible attenuation artifacts. Dedicated QA tools are available from the vendor for patient positioning within the FOV to ensure myocardial coverage, identify detector electronic failure, patient motion, and attenuation artifacts. Data are acquired in a list mode enabling dynamic SPECT radiotracer myocardial blood flow imaging in a tomographic mode. ${ }^{15}$

CZT with multiple single-pinhole detectors (Discovery NM530c, General Electric, Waukesha, WI). In another design, a set of 19 CZT detectors, each $8 \mathrm{~cm} \times 8 \mathrm{~cm}$ in size and coupled to a single-pinhole collimator, is used with optimized geometry for cardiac imaging. ${ }^{3,6}$ The use of simultaneously acquired views improves the overall sensitivity and gives complete and consistent angular data needed for both dynamic studies and for the reduction of motion artifacts. In this system, the detectors and collimators do not move during acquisition, and images are acquired simultaneously by 
all pinholes. Patients are imaged in a supine position with their arms placed over their heads; prone position is also possible. A 2-position supine-prone imaging is often used on this system to mitigate attenuation artifacts. Attenuation correction is possible with attached full-size CT-but this option is not widely used due to significant cost increase of the hardware. Dedicated iterative reconstruction with resolution recovery is utilized for SPECT reconstruction. Traditional rotating projections are not available for this system. For motion correction, the vendor offers tools to track the patient motion from the list-mode data. Dedicated QA tools are available from the vendor to identify detector electronic failure and patient motion. Data are acquired in a list mode enabling dynamic SPECT radiotracer myocardial blood flow imaging in a tomographic mode. ${ }^{16,17}$

Indirect photon detection with solid-state photodiodes (Cardius, Digirad, Suwanee, GA). Another approach is to couple pixelated scintillation crystals to solid-state photodiodes instead of PMTs. This design has been used in a 2- or 3-detector configuration with parallel-hole or fan-beam collimators. Digital logic and software is used to process the signals instead of analog Anger positioning circuits. Fan-beam collimators can also be used for the acquisition of the attenuation maps ${ }^{5}$ by integrated AC system, in which photons from X-ray source are detected by solid-state detectors with a fanbeam collimator operating in high counting-rate mode. With this system, the patient sits upright in a chair that rotates the patient in front of the camera, instead of rotating the camera around the patient.

Conventional cameras with cardio-focal collimators (IQ-SPECT, Siemens Healthineers, Malvern, $P A)$. One vendor offers a cardio-focal collimator (variable focal-length cone-beam) which can be used on a conventional multipurpose camera with optimized organ-of-interest centered acquisition and iterative reconstruction. ${ }^{11}$ With an appropriate orbit, this variable-focus collimator increases the number of detected events from the heart by more than a factor of two in each direction compared to that of a parallel-hole collimator with equivalent resolution, and magnifies the heart while imaging the rest of the torso under traditional conditions. $^{11}$

Other designs. Other system designs have been proposed $^{3}$ but are not presently commercially available, and therefore are not discussed here.

Data sampling. A range of angular projection images is needed to reconstruct tomographic images. The way that SPECT systems acquire the multiple projection images differs significantly. For conventional parallel-hole collimators and Anger cameras or similar detectors, the minimum number of angular samples is discussed in "Acquisition" section.
For other designs, the topic of data sampling may not be so straightforward. Truncation with small FOV detectors may cause artifacts, particularly at the edge of the FOV. Cone-beam and pinhole geometries have less data completeness at distances further away from the central transaxial image slice and show increased image artifacts. Some focused collimator designs may have sufficient sampling in the cardiac region, although not over the rest of the torso. Proper patient positioning is important to ensure that the heart is in the fully sampled region. Vendors typically provide recommended cardiac imaging protocols for their cameras. If modifications of the protocols are to be used, the user should verify the quality of images obtained with the modified protocols with QA phantoms. Studies with a full-scale anthropomorphic phantom would be appropriate for this purpose to simulate imaging under actual conditions.

Patient configuration. Because of the variety in SPECT camera designs, the patient-handling system can differ markedly between SPECT systems. Although this does not impact camera QA directly, the differences in patient configuration and the potential effect on study interpretation should be understood.

The conventional patient-handling system holds the patient horizontally on a level table as the detectors rotate around the patient. Imaging in a supine position is standard and 2-position imaging (supine-prone) is recommended for at least the stress images. ${ }^{18}$ Some dedicated cardiac SPECT systems instead hold the patient in a reclined or upright position. The different direction of gravity relative to the patient may affect the expected patterns of attenuation and the degree and nature of patient motion. If $\mathrm{AC}$ is not used, 2-position imaging can be performed either supine-prone ${ }^{19}$ or upright-supine $^{20,21}$ to provide additional information regarding attenuation-related artifacts. This is especially recommended on the high-efficiency systems with fast acquisition time because the additional scan time required is short. For SPECT systems in which the patient is rotated in the upright position (rather than the detector rotated around the patient), the rotating chair of these cameras must be included in a COR assessment of the QA program.

SPECT systems with photon sources for attenuation correction. Currently, there are two types of transmission tomographic imaging systems for acquiring patient-specific attenuation maps that can be used to correct SPECT images for photon attenuation. The first type, referred to in these guidelines as radionuclide transmission computed tomography (radionuclide-TCT), uses a sealed radioactive source (e.g., Gd-153) with the standard collimated gamma-ray detectors used for SPECT imaging. A variant of this first type is the X-ACT system (Digirad Corp.), which uses lead 
fluorescent X-rays as the source instead of a radioisotope. ${ }^{5}$ The second type of transmission imaging system is a CT scanner (section "SPECT systems with CT for attenuation correction"). The primary differences between these classes of transmission imaging systems include the type of radiation used and the photonemission rate that dictates the quality-control (QC) protocols that are required. The radiation used in radionuclide-TCT is monoenergetic gamma rays, while the radiation with $\mathrm{CT}$ is polyenergetic. Also, the intensity of the radiation flux from CT is much higher than that found with radionuclide-TCT. CT images can be acquired on the order of seconds to a few minutes depending on the X-ray tube strength, while radionuclide-TCT may require 4 to 10 minutes.

As the radiation flux is much lower with radionuclide-TCT, images from these systems have higher noise content than CT images. Also, scatter photons from the emission source can affect the transmission image obtained from radionuclide-TCT but have negligible effect on CT images due to the large difference in photon count rates. The higher transmission image noise and interference between emission and transmission images make the radionuclide-TCT QC procedure slightly more involved, and will be outlined separately from the QC for CT systems.

An advantage of radionuclide-TCT systems is that they use the same detectors for both emission and transmission imaging, and thus the mechanical coregistration of the transmission and emission data is not an issue. Patient motion can still cause misregistration; hence, it is still important to assess each clinical study for registration. A disadvantage of radionuclide-TCT systems is that more time is required to acquire the transmission image with increasing age of the sealed source, and the sealed sources require periodic replacement.

SPECT systems with CT for attenuation correction. Consistent with trends in positron emission tomography (PET)/CT systems, hybrid SPECT/CT systems have evolved. These combined systems, in practice, demonstrate a range of capability and integration. The $\mathrm{CT}$ subsystems range from units suitable for use in anatomical localization and AC to multislice (16 slices or more) systems capable of CT angiography and producing diagnostic quality $\mathrm{CT}$ images. The SPECT detectors in SPECT/CT systems do not differ in any significant way from those of standalone SPECT systems. These systems may be viewed, from a protocol perspective, as standalone systems where an emission study is followed or preceded by a CT scan for AC. Depending upon the number of $\mathrm{CT}$ slices acquired, the CT scanner may be used, as with standalone CT scanners, for CT angiography and calcium scoring.
The images can then be analyzed independently or in three-dimensional (3D) image registration with the SPECT images, depending on the type of study. Coronary calcium score scans from standalone multislice CT have also been successfully used for AC of ${ }^{99 \mathrm{~m}}$ Tc SPECT MPI. ${ }^{22}$

\section{QC Procedures}

The overall SPECT image quality depends on a combination of the detector performance, collimator dimensions, system design, and image reconstruction algorithm (section "Processing"). Whereas conventional SPECT cameras have similar design and performance (depending on the collimator specifications), other systems with nonconventional designs may have much different performance. Laboratories with multiple SPECT systems of different design should ensure that the consecutive rest-and-stress SPECT exams for the same patient are performed on the same or equivalent cameras.

Basic image quality tests, such as spatial resolution, sensitivity, and energy resolution are performed with simple phantoms or procedures. They generally test one or more components of the system, and, although they provide valuable information, they do not provide a full assessment of the total system performance. Performance assessment is complicated by the fact that both resolution and sensitivity vary with respect to the detector orbit, the location in the FOV, and the presence of activity outside the region-of-interest (ROI). Larger fillable phantoms that contain an extended source, coupled with a specific imaging test (e.g., hot or cold lesion contrast, uniform volume, myocardial defect detection, etc.) will provide an overall system evaluation and are valuable in assessing performance between systems. For these reasons, tests with realistic fillable phantoms are essential for performance characterization, and should also be included in routine QC.

Camera acceptance. It is recommended that the National Electrical Manufacturers Association (NEMA) performance measurements (NU 1-2012) ${ }^{23}$ be made before accepting the SPECT camera to ensure that it is operating at the level specified by the manufacturer. ${ }^{24}$ Many of these tests can be performed by the company supplying the SPECT camera, but this represents a potential conflict of interest, so it is recommended that acceptance testing be verified independently by a qualified medical physicist. ${ }^{25}$ Overall camera performance should also be assessed with a multipurpose plexiglass phantom immediately following or as part of acceptance testing to establish baseline reference images. 
For new technologies that may not yet be addressed by the NEMA specifications, ${ }^{26}$ these guidelines are intended to indicate the essential technical aspects that should be assessed with respect to camera function in conjunction with nuclear cardiology imaging. Because imaging systems can vary considerably with the optimal manner to perform specific tests, this document should be used as a guideline only and is not intended to replace the recommendations by manufacturers of specific models of imaging equipment. The performance of the new dedicated cameras should meet the same minimum standards expected of conventional Anger-style cameras with parallel-hole collimation.

SPECT QC procedures. Appropriate QC procedures (Table 3) are necessary to ensure images of the highest possible technical quality for the equipment are used, and thus allow the best possible diagnostic service to the patient population. ${ }^{27}$ Please note that the frequency of testing requirements may vary from one state to the next, so it is important to verify that the schedule followed by an individual institution is consistent with that institution's radiation license. For instance, some states require weekly bar phantom scans be performed for all gamma cameras.

This subsection outlines the protocols and test frequency for Anger cameras. The manufacturers for novel cameras, not based on Anger technology, should provide comparable protocols for their systems to ensure the optimal operation for clinical use. If the manufacturer does not provide an equivalent test, and the frequency to the tests outlined in this section, the existing guideline protocols and frequencies should be enforced. For manufacturer protocols and frequencies, the manufacturer should provide data that ensures the measurement has defined tolerance levels (e.g., uniformity has a defined threshold for acceptance). With respect to frequency, these guidelines suggest the expected frequency. The manufacturer should provide data supporting a frequency that is less frequent than what is proposed in these guidelines.

Energy calibration check. This test is performed to verify that the energy window used for counting photons is aligned with the photopeak energy. The full useful field of view (UFOV) of the camera should be illuminated by the source used. The test can be performed intrinsically (with no collimator on the camera) using a radioactive point source at a distance greater than 5-UFOV diameters away from the detector surface, or extrinsically (with a collimator on the camera) using a uniform sheet source. The test consists of either manually or automatically verifying, and adjusting as needed, the position of the pulse height analyzer's energy window with respect to the photopeak energy of the source being measured. Window verification should be done even on automated systems where there are single buttons or computer protocols to select for each energy; even in these automated systems, the energy windows tend to drift. Some systems allow for window offsets to correct for these drifts. The calibration test should indicate for each camera head whether the camera's automatic calibration circuitry is working properly, whether the peak appears at the correct energy, and whether the shape of the spectrum is correct. Energy resolution of the detector at the FWHM can also be measured using the same technique (Figure 3). Annual testing of energy resolution is required by some accrediting bodies. If cost, time, and the equipment permit, an electronic or hardcopy screen capture of the spectra with the superimposed energy window should be taken and stored. Care must be taken to adjust the energy calibration appropriately when using the camera for dual-isotope imaging with ${ }^{99 \mathrm{~m}} \mathrm{Tc}$ and ${ }^{201} \mathrm{TI}$ imaging, as

Table 3. Quality control procedures for SPECT systems

\begin{tabular}{|c|c|c|}
\hline Test & Priority & Frequency \\
\hline Acceptance testing per NEMA & Recommended & Upon delivery, major hardware upgrade \\
\hline Energy calibration check & Mandatory & Daily \\
\hline Uniformity test & Mandatory & Daily \\
\hline Resolution and linearity & Recommended & Manufacturer's recommendation \\
\hline Sensitivity & Optional & Manufacturer's recommendation \\
\hline Center-of-rotation and & Mandatory & Manufacturer's recommendation \\
\hline Multidetector registration & & $\begin{array}{l}\text { After camera servicing, after power } \\
\text { surges or outages, after hardware } \\
\text { and software upgrades }\end{array}$ \\
\hline Uniformity calibration & Mandatory & Manufacturer's recommendation \\
\hline Total performance & Recommended & Quarterly \\
\hline
\end{tabular}

NEMA, National Electrical Manufacturers Association 


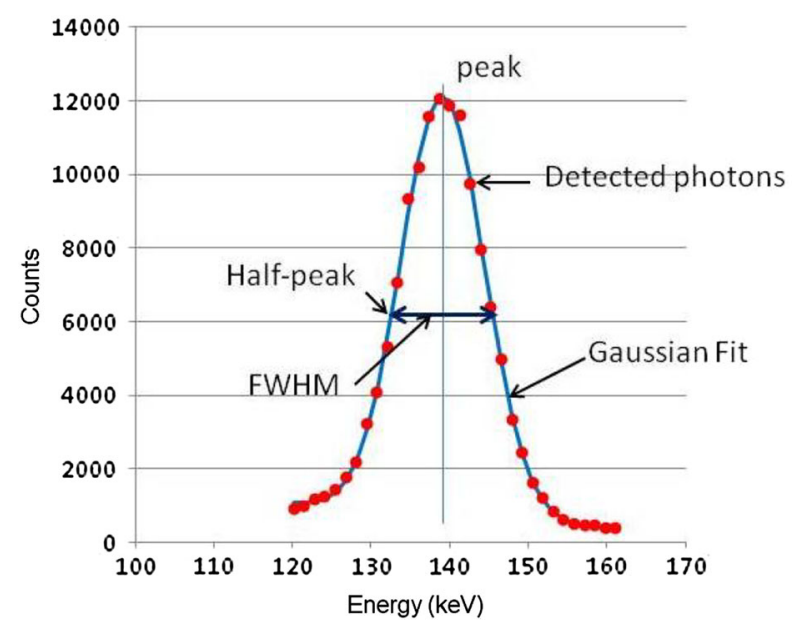

Figure 3. Measuring Energy Resolution. The energy resolution is measured as the full width at half maximum (FWHM) of the detected energy spectrum, of ${ }^{99 \mathrm{~m}} \mathrm{Tc}$ with photopeak energy at $140 \mathrm{keV}$, expressed as a percentage of the photopeak energy.

alignment of the energy window for one isotope may influence the alignment of the second isotope's energy window.

Uniformity test. Uniformity testing is performed to verify that the camera's sensitivity response is uniform across the detector's face. Some manufacturers recommend this test be performed intrinsically using a point source, while others recommend this test be performed extrinsically using a sheet source, usually of Cobalt-57. The test consists of exposing the camera to a uniform source of radioactivity, a process commonly referred to as "flooding" the detector. If performed intrinsically, a radioactive point source is positioned at a distance at least five times the crystal's UFOV from the center of the detector (Figure 4). The test is usually performed immediately following verification of the energy calibration of the detector. The point source should consist of a small volume (approximately 0.5 $\mathrm{mL}$ ) and low activity such that the measured count rate is between 10 and 20 kilo counts per second (kcps) (7 to 11 megabecquerel $[\mathrm{MBq}]$ ). For large rectangular cameras (where the point should be 7 to 8 feet [ 2 to 2.5 meters] away), 20 to $25 \mathrm{MBq}$ may yield a similar count rate. In some cameras, obtaining intrinsic flood fields can be difficult. Some manufacturers provide software to correct for non-uniformities due to the necessity of having a point source closer than 5-UFOV diameters. Because of these difficulties, it may be more practical to perform this test extrinsically using radioactive sheetflood sources. For some older systems, a lead ring should be used to shield the outermost tubes from the

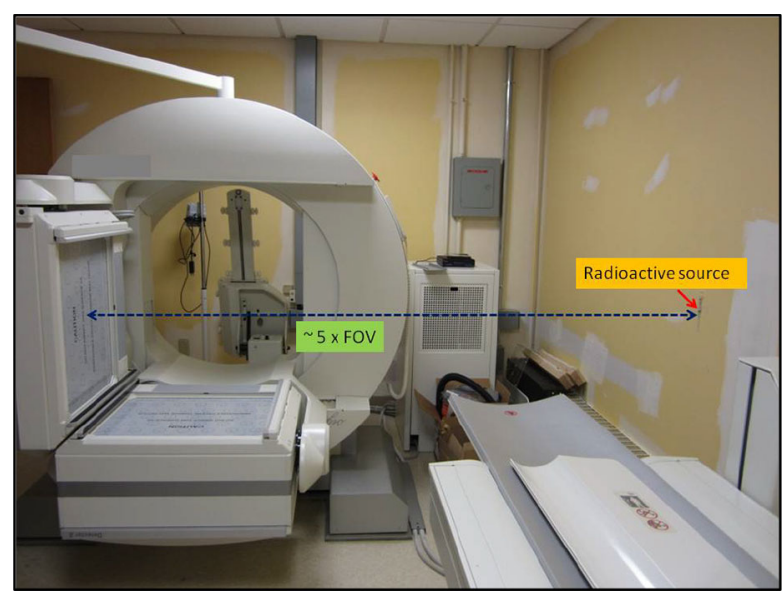

Figure 4. Test setting for Intrinsic Uniformity. A syringe was filled with $20-25 \mathrm{MBq}$ of ${ }^{99 \mathrm{~m}} \mathrm{Tc}$ in $0.5 \mathrm{ml}$, then positioned $\sim 3$ meters away from the detector.

radiation to prevent edge packing. Flood images that will be inspected visually should be acquired as $256 \times$ 256 matrices for 3 million counts ( 5 million counts for larger rectangular detectors). Digitized flood fields should be stored. Flood images used for calculations of uniformity require two to three times more counts to reduce statistical noise. The recommended number of counts is at least 4,500 counts $/ \mathrm{cm}^{2}$ (e.g., 5.7 million counts for a 400-mm diameter circular detector). For accurate quantification of uniformity, the NEMA recommends acquiring a minimum of 10,000 counts for the center $(6.4 \mathrm{~mm})$ pixel of the flood image, or approximately 50 million total counts for $40 \mathrm{~cm} \times 50 \mathrm{~cm}$ detectors. $^{24}$

The flood images should be examined each day for each detector prior to use to verify the detectors are properly peaked and the floods are uniform. In addition, several parameters are quantified from the flood images, which should be computed and the results recorded as part of the usual QA procedures. In the event of power shortages and power outages, the process of energy calibration and flooding the detectors should be performed again to ensure proper function before resuming patient imaging. If the camera has been without power for a long period of time, it should be allowed time to stabilize prior to energy calibration and uniformity testing. Two uniformity parameters are computed: integral uniformity and differential uniformity. If the flood images are acquired in a larger matrix size, the pixel matrix should be reduced to 64 x 64 by summing or averaging pixels prior to uniformity calculation. Integral uniformity is a global parameter measuring 
Table 4. Typical uniformity and intrinsic resolution for scintillation (or Anger) cameras

\begin{tabular}{|c|c|c|}
\hline Parameter & Standard & Preferred \\
\hline Integral & $<5 \%$ & $<3 \%$ \\
\hline Differential uniformity & $<5 \%$ & $<3 \%$ \\
\hline Intrinsic resolution (FWHM) & $<6 \mathrm{~mm}$ & $<4 \mathrm{~mm}$ \\
\hline
\end{tabular}

Note: These values are specific to Anger camera systems using a 3/8-in [9.5 mm] Nal(TI) crystal

FWHM, full width at half maximum

uniformity over an extended area of the detector, expressed as follows:

Integral uniformity $=100 \%(\operatorname{Max}-\operatorname{Min}) /(\operatorname{Max}+\operatorname{Min})$

Max is the maximum count and Min is the minimum count found in any pixel within the specified area. Differential uniformity is a regional parameter that measures contrast over a small neighborhood. This measurement is performed using all $5 \times 5$ pixel areas in both the $x$ and $y$ directions, expressed as follows:

Differential uniformity $=100 \%$ (Largest deviation $[$ Max

$$
-\operatorname{Min}]) /(\operatorname{Max}+\operatorname{Min})
$$

Typical uniformity values are given in Table 4 .

It should be noted that manufacturers vary as to their recommendations regarding the ability of a particular camera to use a flood field collected at one energy (e.g., $140 \mathrm{keV}$ for ${ }^{99 \mathrm{~m}} \mathrm{Tc}$ ) to correct the field of data acquired at a different energy (e.g., $70 \mathrm{keV}$ for $\left.{ }^{201} \mathrm{Tl}\right){ }^{28}$ For some Anger cameras it may be essential to acquire flood fields separately for ${ }^{201} \mathrm{Tl},{ }^{123} \mathrm{I},{ }^{131} \mathrm{I}$, and so on. Failure to apply an adequate overall flood-field correction is seen most strikingly as concentric ring artifacts on transverse images of the uniform section of a SPECT Performance Phantom. ${ }^{27}$

High-count flood-field uniformity corrections. Anger cameras use stored high-count flood-field correction maps to compensate for variations in sensitivity across the detector FOV before reconstruction. Manufacturers vary considerably as to their recommended schedule and means of acquiring these highcount corrections. It has recently been noted that some cameras may not require the acquisition of extrinsic floods more often than annually to verify collimator integrity ${ }^{10}$ and all uniformity corrections should be acquired intrinsically, so long as the camera is correctly tuned. ${ }^{24}$ For many systems, collimators are sufficiently designed and manufactured so they do not degrade SPECT uniformity. Therefore, as with any of the procedures discussed in these guidelines, it is always important to follow the manufacturers' recommended QA protocols. To correct for uniformity, a high-count (minimum 60-million but frequently 100-million or higher count) flood image is acquired for each detector and stored. It may be necessary to obtain separate correction maps for each collimator and isotope combination that will be used clinically, and manufacturer recommendations should be followed. Using correction maps corresponding to a different energy window or collimator can result in incorrect uniformity compensation.

Resolution and linearity test. This test is performed to document spatial resolution and its change over time as well as the detector's ability to image straight lines. The test consists of imaging a flood source intrinsically through a spatial resolution test phantom. The source can be either a point or sheet source. The activity of the source should be such that the measured count rate is between 10 and $20 \mathrm{kcps}$. Most commercially available bar phantoms are suitable for this test. These include the parallel-line-equal-space bar phantoms and orthogonal hole or 4-quadrant phantoms (Figure 5). If the 4-quadrant phantom is used, each time the test is conducted, the phantom should be rotated $90^{\circ}$, so every fifth time the test is done, the pattern position repeats. Bar-phantom images should be recorded and stored. These images are evaluated for the appearance of the straight lines and for intrinsic spatial resolution. The change in resolution is assessed by documenting the smallest bars that are discerned. Spatial resolution as measured by the FWHM may be approximated by multiplying 1.7 times the smallest bar size seen. ${ }^{27}$

For imaging systems not based on the Anger-camera technology, the manufacturer should provide a QC procedure to ensure the resolution and linearity stability is maintained by the system.

The test should be performed weekly or as recommended by the manufacturer or local regulatory agency.

Sensitivity test. This test is performed to document the sensitivity of the camera and, more importantly, the change in sensitivity over time. The test consists of calculating camera sensitivity (expressed in terms of counts per minute [cpm] per MBq) of a known source, calibrated with a dose calibrator. The point source should always be located at exactly the same distance in front of the camera for repeat measurements. A convenient means of measuring sensitivity changes is by recording the time it takes to acquire the preset counts for an intrinsic (or extrinsic, if more practicable) flood source.

A syringe or flask filled with a known radioactivity $\left(\mathrm{A}_{0}\right)$ with a count rate less than $20 \mathrm{kcps}$, is placed close to the camera head and imaged for one minute through 


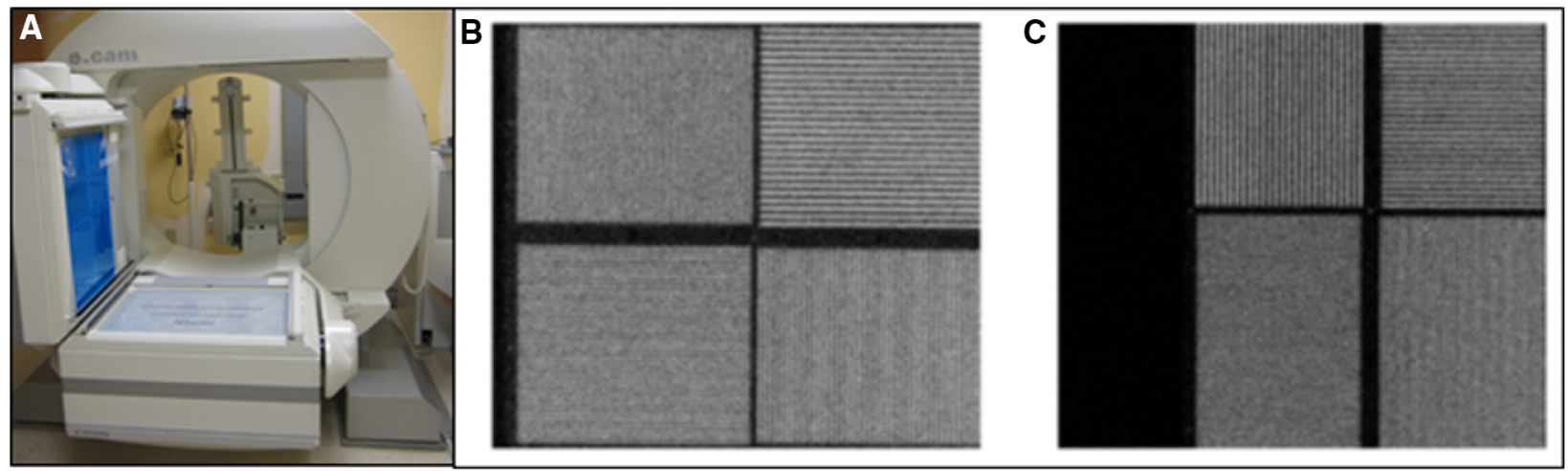

Figure 5. Bar-phantom images and a set-up for intrinsic resolution test. (A) The bar phantom with $2.0,2.5,3.0$, and $3.5 \mathrm{~mm}$ spacing was placed directly on the detector without collimator and a syringe filled with radioactivity was positioned away from the detector as shown in Fig. 2. (B) Image of bar phantom. (C) The phantom was rotated $90^{\circ}$.
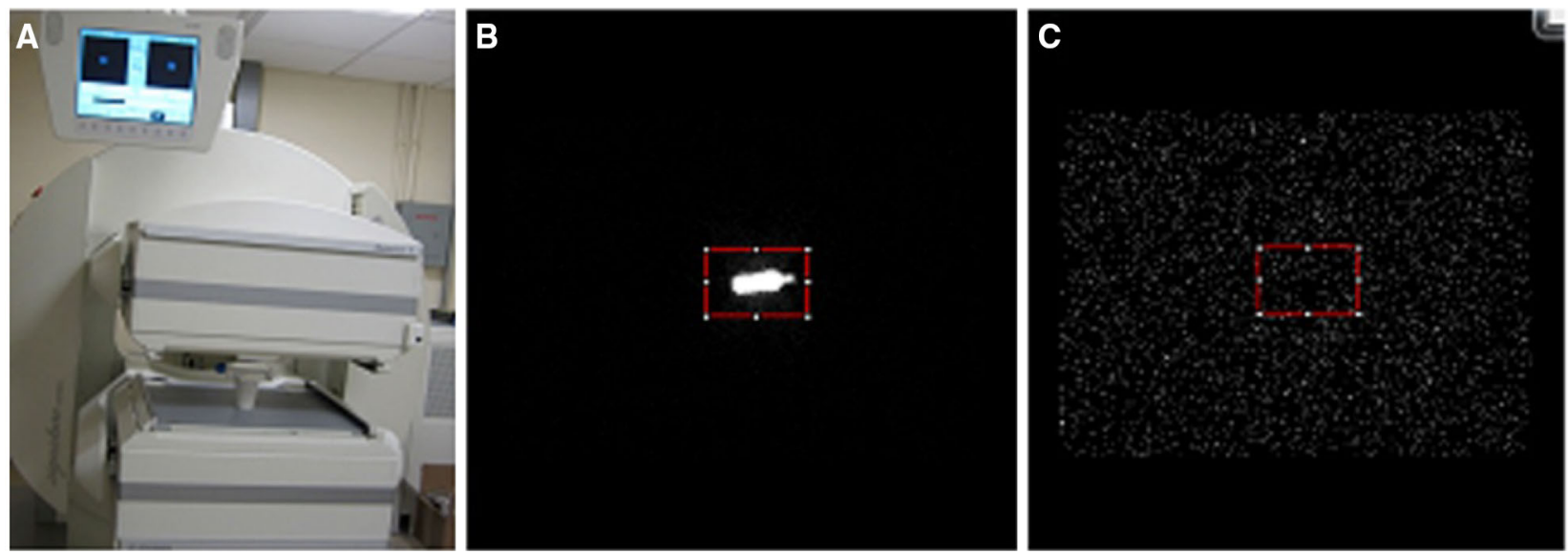

Figure 6. Estimating planar sensitivity. (A) Two detectors are set up $20 \mathrm{~cm}$ apart and a syringe filled with radioactivity $\left(\mathrm{A}_{0}\right.$, with $<20$ kilo counts/s) is placed in the middle using a supporting cup with minimal attenuation. (B) Image of the radioactive source with a ROI for the total counts $\left(\mathrm{C}_{\text {syringe }}\right)$, and $(\mathrm{C})$ image of the background with the same ROI for the background counts $\left(\mathrm{C}_{\mathrm{bkg}}\right)$. Planar sensitivity is calculated as $\left(\mathrm{C}_{\text {syringe }}-\mathrm{C}_{\mathrm{bkg}}\right) / \mathrm{A}_{0}$.

an energy window recommended by the manufacturer (Figure 6). The total counts within the ROI is then recorded as $\mathrm{C}_{\text {syringe. }}$ The source is removed, and background radiation is acquired for one minute. The background counts within the same ROI is estimated as $\mathrm{C}_{\mathrm{bkg}}$. The planar sensitivity is calculated using $\left(\mathrm{C}_{\text {syringe }}\right.$ $\left.-\mathrm{C}_{\mathrm{bkg}}\right) / \mathrm{A}_{0}$

Center-of-rotation calibration. An alignment error between the center of the electronic matrix of the camera and the mechanical COR can potentially result in a characteristic "doughnut" (if a $360^{\circ}$ orbit and a point source are used) or "tuning fork" artifact (if a $180^{\circ}$ orbit is used) in the transverse images. ${ }^{28}$ The effects are most evident when the error is greater than two pixels in a $64 \times 64$ matrix. Errors less than this reduce spatial resolution and image contrast through blurring of the image and cause significant artifacts (particularly at the apex). ${ }^{29}$ The accuracy of COR alignment should be checked weekly for each camera head, unless indicated otherwise by the manufacturer. In some systems, this means that two separate acquisitions are required: one for each detector (with the other detector disabled). Many manufacturers require that a specific protocol be followed for the determination and 
recalibration of the COR. While some manufacturers limit the COR calibration to service engineers, all systems should be checked for correct COR calibration. If no specific COR acquisition protocol is recommended by the manufacturer, the COR can be determined through the acquisition of a point source of activity (18 to $37 \mathrm{MBq}$ ) on the patient table 10 to $20 \mathrm{~cm}$ away from the axis of rotation. SPECT data are acquired over $360^{\circ}$ with equally spaced projections with a circular orbit. The same angular orientation, collimation, zoom, matrix size, and energy window employed for the patient study should be employed for the COR acquisition. Five to ten seconds per frame for 64 views over $360^{\circ}$ is sufficient. Center-of-rotation correction values for each orbit are then computed, stored in the computer, and used to realign the projection data before reconstruction. The $\mathrm{COR}$ is measured as the average position of the point source in two projections that are acquired $180^{\circ}$ apart. The COR error is the transverse distance of the measured COR from the center of the projection. It is essential that COR errors be checked for each collimator that is to be used clinically. ${ }^{30}$ It is recommended that these measurements be performed weekly.

New COR calibrations should be performed after servicing of the camera, after power surges or outages, and after hardware and software upgrades. It is important to verify that the correct COR information is applied following service of any type by simply repeating the acquisition of new COR information, and then verifying that a tomographic acquisition of a point source produces a transaxial image correctly that is also a point source.

Multipurpose plexiglass phantoms. It is strongly recommended by NEMA that acquisition and reconstruction of a multipurpose plexiglass phantom be performed quarterly at a minimum ${ }^{31}$ to assess overall image performance. For facilities intending to pursue laboratory American College of Radiology (ACR) accreditation, performing these tests is mandatory. It should be noted that if accreditation is sought from the $\mathrm{ACR}$, it is required to submit acceptable SPECT phantom images for both ${ }^{99 \mathrm{~m}} \mathrm{Tc}$ and ${ }^{201} \mathrm{Tl}$ acquisitions. This test should be performed whenever the system has undergone significant servicing or when any of the daily tests exceeds recommended tolerances.

The multipurpose phantoms are cylindrical or elliptical water baths into which radioactivity is injected. They contain regions with solid spheres of different sizes, regions with solid rods or bars of different sizes alternating with radioactive water, and regions containing only radioactive water. ${ }^{32}$ The different phantom regions are used to determine the $3 \mathrm{D}$ contrast, resolution, and uniformity of the camera respectively. Low-noise projection data containing at least 30 million counts should be acquired using " "fine", sampling (generally 128 x 128 matrices and 128 projections over $\left.360^{\circ}\right){ }^{27}$ Acquisitions are performed using typical ${ }^{99 \mathrm{~m}} \mathrm{Tc}$ energy settings, with detectors positioned as close to the phantom as is feasible throughout a $360^{\circ}$ acquisition, so as to optimize spatial resolution. ${ }^{33}$ Systemic problems that can be revealed in this fashion include suboptimal energy resolution through failure to display adequate contrast in images of the spheres, potential COR problems through loss of resolution in images of the solid-rod region, and inappropriate or inadequate flood-field corrections through the appearance of anomalous concentric rings in images of the uniform region. These tests should be performed quarterly, as well as following major equipment repairs and installation of new software, to verify the overall ability of the hardware and software to perform tomographic reconstructions correctly.

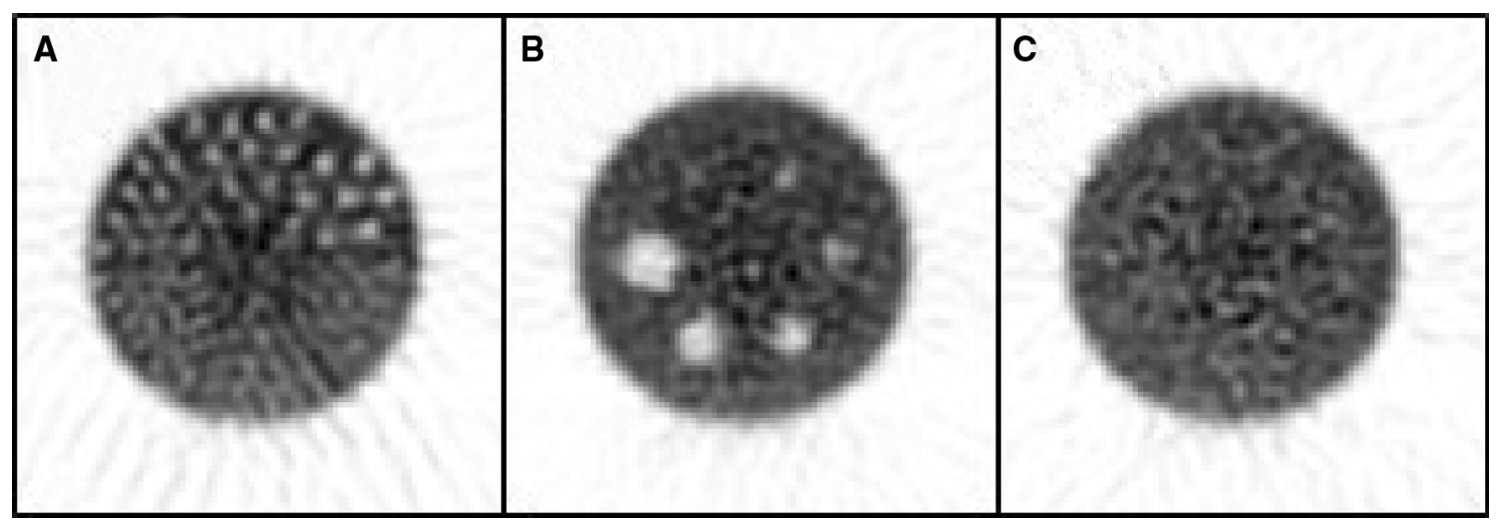

Figure 7. Example images from a multipurpose SPECT phantom. (A) Images of the solid-rod region indicate spatial resolution. (B) Images of the spheres are used to assess contrast (energy resolution). (C) Images of the uniform water region evaluate artifacts and uniformity corrections. 
Figure 7 shows example slices for resolution, contrast, and uniformity of the SPECT phantom.

When used in conjunction with reference images acquired during acceptance testing, these quarterly tests can be helpful in signaling the point at which the manufacturer's service representatives should be called to further diagnose the causes of significant degradation of $3 \mathrm{D}$-system performance and to remedy these problems. ${ }^{24}$

Radionuclide TCT QC procedures. Tests for radionuclide-TCT are tabulated in Table 5.

Energy Calibration Check. Similar to the energy calibration check for emission studies, it is necessary to verify that the energy window used to acquire transmission data aligns with the photopeak of the transmission isotope. For some systems, this may necessitate manually opening the shutter to the transmission source. If this is not possible, a quick "blank" scan can be acquired to verify that transmission photons are being properly counted.

Transmission source mechanics. When patients are not being imaged, the transmission source is shielded and, on systems where the source translates across the FOV, left in the "parked" position. When a patient is imaged, the shutter used to shield the source is opened, allowing transmission photons to be directed toward and through the patient. In some systems, the source will then translate axially or transversely across the FOV for each projection. To verify the operation of the source shutter and translating mechanics, a reference "blank" transmission scan should be acquired. This scan is required for all TCT protocols and is recommended to be acquired weekly and possibly daily prior to the first use of the system for that day. The frequency of this test will depend on the half-life of the isotope of the transmission source and the stability of the TCT system. Follow the manufacturer's recommended acquisition protocol for acquiring a transmission blank scan. When complete, visually inspect planar images and check for artifacts (e.g., focal cold spots, bands of missing data, axial discontinuities). A common misconception is that

Table 5. QC procedures for radionuclide TCT systems

\begin{tabular}{lcc}
\hline \multicolumn{1}{c}{ Test } & Requirement & Frequency \\
\hline $\begin{array}{l}\text { Energy calibration } \\
\text { check }\end{array}$ & Mandatory & Daily* \\
$\begin{array}{l}\text { Transmission source } \\
\text { mechanics }\end{array}$ & Mandatory & Daily* \\
$\begin{array}{l}\text { Source strength } \\
\text { QC, quality control; } T C T \text {, transmission computed tomography } \\
\text { *Or as recommended by the manufacturer }\end{array}$ & Mandatory & Monthly* \\
\hline
\end{tabular}

the blank scan should be uniform, similar to uniformity floods. Stringent uniformity indices of $\pm 10 \%$ are not reasonable for the blank scan. Rather, the blank scan should be inspected to ensure there are no gross nonuniformity artifacts (e.g., holes or bands of pixels with no counts). For scanning-source systems, the blank scans should not show discontinuities or abrupt changes in pixel intensity in the direction of the scanning source. The presence of these artifacts is consistent with improper scanning-detection alignment and should be checked by a service engineer.

Source strength. For systems using a Gd-153 transmission source, photons collected in the transmission window consist of primary transmission photons and scattered photons (cross-talk) from the emission radiotracer. The ratio of these components, transmission and cross-talk, is referred to as the transmission-to-cross-talk ratio (TCR). This TCR value depends on the transmission source strength, the injected radio-pharmaceutical, the injected activity, and the body habitus. Transmission source decay, higher injected activities, and larger body sizes all tend to decrease the TCR value. Lower TCR values result in reconstructed attenuation maps with increased bias and noise. Because the TCR value will decrease as the source decays, its behavior should be trended over the life of the source, which can guide the user as to when the sources should be replaced. This QC protocol should be performed at least monthly, with the baseline scan being performed when the system is installed or the transmission sources have been replaced. If the user suspects problems with the transmission system, a test scan of an attenuation phantom should be performed and evaluated immediately prior to using the system for patient imaging. For cardiac imaging, an anthropomorphic chest phantom is preferred for this test, as it is a better representation of the heterogeneous attenuation present in patient studies. If the chest phantom is not available, a cylinder phantom can be used. Accuracy of the measured attenuation coefficients should be assessed as per manufacturer recommendations.

CT QC procedures. A comprehensive annual evaluation of CT system is required by state or federal regulations and for accreditation. Both the ACR and American Association of Physicists in Medicine (AAPM) have published $\mathrm{CT}$ testing procedural guidelines. $^{34,35}$

For SPECT AC, CT number calibration and uniformity must be checked by a qualified person defined by regulatory or accreditation agencies.

CT number calibration. The reconstructed CT image must exhibit accurate, absolute CT numbers in Hounsfield Units (HU). This is critical for the use of CT images for SPECT AC, because the quantitative CT values are transformed into the attenuation coefficients 
for the SPECT radiotracer, usually via a bilinear or trilinear function with one hinge at or near the $\mathrm{CT}$ value for water. ${ }^{36}$ Any errors in CT numbers will be propagated as errors in the estimated attenuation coefficients for the radiotracer, which in turn will adversely affect the attenuation-corrected SPECT values. CT system calibration is performed with a special calibration phantom that includes inserts of known CT numbers. This calibration is done by the manufacturer's field service engineers. The CT calibration is then checked daily with a water-filled cylinder, usually $24 \mathrm{~cm}$ in diameter provided by the manufacturer. The manufacturer will specify the acceptable range of $\mathrm{HU}$ values measured for water. In practice, if the error is greater than 5 to $10 \mathrm{HU}$ (i.e., different than the anticipated value of $0 \mathrm{HU}$ ), the CT system is considered to be out of calibration. An air calibration (or blank-scan acquisition) can be performed to determine if this corrects the error. If it does not, the manufacturer's field service engineer must be called. Calibration must be checked by a qualified person on an annual basis, or after any major repair or calibration.

Field uniformity. The reconstructed CT image must exhibit uniform response throughout the FOV. In practice, this means that a reconstructed image of a uniform water-filled cylinder must itself demonstrate low variation in $\mathrm{CT}$ number throughout this image. In practice, small square or circular ROIs are placed at $90^{\circ}$ intervals near the edge of the cylinder image, and the mean CT number in each ROI is compared to that from a region in the center of the phantom; the maximum difference in mean region CT number should not exceed 5 $\mathrm{HU}$ or the value specified by the manufacturer. Nonuniformities greater than this may produce sufficient quantitative inaccuracies to affect SPECT AC based on the CT image.

For combined SPECT/CT units, users should consult the manufacturer regarding the specific manner and frequency of routine CT QA with which tests should be performed for the CT component of their SPECT/CT device. Typically, prior to patient imaging, a tube warmup and air scan are performed daily followed by a waterphantom scan. The water-phantom image is then used to check for accuracy and standard deviation of the water CT number, and for artifact evaluation.

Combined TCT and SPECT QC procedures. In addition to the independent $\mathrm{QC}$ tests for the SPECT and TCT portions of the combined system, it is necessary to perform tests that assess their combined use. Table 6 lists recommended QC procedures for combined SPECT/CT units.

Registration. The reconstructed SPECT and CT images must accurately reflect the same 3D locations (i.e., the two images must be in registration). Such
Table 6. Combined TCT/SPECT QC procedures

\begin{tabular}{cc}
\hline \multicolumn{1}{c}{ Test } & \multicolumn{1}{c}{ Requirement } \\
\hline Registration & $\begin{array}{c}\text { Mandatory for X-ray TCT } \\
\text { systems } \\
\text { Mandatory }\end{array}$ \\
AC accuracy & \\
\hline
\end{tabular}

$A C$, attenuation correction; $T C T$, transmission computer tomography; $Q C$, quality control

registration is often difficult because the SPECT and CT subsystems of most commercial combined SPECT/CT systems are not coincident (i.e., different detectors are used for each subsystem, so the SPECT and CT 'slices' are not in the same plane). In fact, because the bed must travel different distances into the gantry to image the same slice in the patient for SPECT versus CT, there is ample opportunity for misregistration via $\mathrm{x}, \mathrm{y}, \mathrm{z}$ misalignment of bed motion-or, of perhaps even greater concern, because of differential "bed sag" for the SPECT and CT portions, depending on the table design.

In addition, electronic drift can influence the "position" of each image, so calibrations for mechanical registration can become inaccurate over time. Thus, it is imperative to check SPECT-to-CT registration on an ongoing basis. This is usually performed with a specific phantom or jig containing an array of point sources visible in both SPECT and CT.

Errors in co-location in the fused SPECT-CT images are assessed, for example, by means of count profiles generated across transaxial slices. Such errors, after software registration corrections, should be less than 1 to $3 \mathrm{~mm}$, as specified by the manufacturer. It is important to image this registration jig in a number of positions along the bed. It may also be helpful to place a weight on the end of the bed to produce some bed sag and repeat the assessment.

The SPECT emission images are obtained over several minutes, averaging the heart motion over many respiratory cycles, while the $\mathrm{CT}$ transmission image is obtained much more rapidly with little, if any, respiratory-motion averaging, depending on the CT subsystem. The differences in acquisition duration along with differences in image resolution of the SPECT and CT images can also increase the chance of malalignment between the transmission and emission images.

For radionuclide-TCT systems, the same detectors are used for both emission and transmission and mechanical registration of the system is not needed. Transmission and emission images should still be 
evaluated for patient studies as patient motion may lead to misregistration in some cases.

Note: The above considerations are in addition to the patient-specific alignment QC clinically necessary to assess possible patient or respiratory motion (not described here).

Attenuation correction accuracy. The overall AC performance should be assessed using a corrected SPECT image of a large water-filled cylinder containing a uniform activity concentration. Errors in TCT-toSPECT AC are usually manifest as a SPECT image, which does not have a "flat" profile from edge to center (i.e., the activity at the edge is either too high or too low relative to that at the center of the phantom). If an absolute camera calibration is available, the accuracy of the reconstructed activity concentration can also be assessed, though this measurement is additionally dependent on the accuracy of the scatter correction.

\section{Recommendations for SPECT Instrumentation, Quality Assurance, and Performance}

- ASNC recommends the use of SPECT equipment that meets industry standards and the implementation of quality assurance procedures as described in this document to optimize image quality and patient care.

\section{ACQUISITION}

Acquisition protocols and parameters for standard SPECT MPI for Anger-camera technology (Tables A1A3) and for high-efficiency CZT systems (Tables A4A6) are presented in the Appendix.

The majority of myocardial perfusion SPECT protocols utilize the lowest activity possible of ${ }^{99 \mathrm{~m}} \mathrm{Tc}$ based agents for the specific camera system and acquisition duration; however, ${ }^{201} \mathrm{Tl}$ is used occasionally for the assessment of myocardial viability and in instances of ${ }^{99 \mathrm{~m}} \mathrm{Tcshortage}$. Dual-isotope protocols with rest ${ }^{201} \mathrm{Tl}$ and stress ${ }^{99 \mathrm{~m}} \mathrm{Tc}$ should be avoided due to the higher patient radiation exposure and differential spatial resolution for the two radiotracers. Whenever feasible (see section "Stress-first/stress-only Imaging"), stress-first imaging is recommended ${ }^{37,38}$; otherwise, depending on the clinical question (see section "Patient centered MPI'), a one-day, low-dose rest and high-dose stress protocol, a one-day, low-dose stress and high-dose rest protocol, or a two-day protocol with equal radiotracer activity for stress and rest can be considered. ${ }^{39}$ It is important to recognize that no single protocol is optimal for every patient, and laboratories should aim to implement patient-centered imaging rather than performing the same protocol for every patient (see "Patientcentered MPI").

For each protocol, the acquisition parameters are discussed below and parameters and values are listed separately for the stress and rest portions in Tables A1A6 (see Appendix). Implementation of these acquisition parameters have been shown to provide acceptable images of good quality for routine clinical interpretation and quantitation. The application of algorithms for AC, scatter correction, resolution recovery, collimator-specific geometric response characteristics, and camera response depth-dependence have resulted in similar or superior image quality to the conventional parameters outlined below (see "Processing"'). ${ }^{40}$ The same acquisition parameters and values apply to the SPECT acquisitions for most dedicated SPECT and hybrid SPECT/CT systems. The CT transmission scans are generally performed free-breathing without electrocardiographic (ECG) gating (see sections "Recommendations for SPECT MPI acquisition" and "Attenuation Correction").

The development of high-efficiency, solid-state cameras utilizing CZT detectors has allowed for the use of lower radiotracer activity and shorter acquisition times for MPI. Tables A4-A6 (see Appendix) summarize the recommended acquisition parameters for the two most commonly available high-efficiency CZT systems. For some of these systems, the acquisition can be guided by a goal of a specific number of cardiac counts, rather than a set duration of scanning. Thus, the CZT-detector camera systems are not only more efficient capturing emission counts, but acquisition time can be more efficiently tailored to the patient. Quantification of myocardial flow reserve (MFR) is possible with SPECT cameras, but is facilitated with high-efficiency systems, allowing fast acquisitions. ${ }^{15}$ Tailored MPI protocols using ${ }^{99 \mathrm{~m}} \mathrm{Tc}$-based agents, and imaging protocols specific to the clinical question and to SPECT instrumentation are recommended. ${ }^{39}$

\section{Activity}

The recommended isotope activity depends on several parameters including the following: patient body habitus, study protocol (stress first, 1 or 2 day), scanner type, software type, and radiotracer used. The radiotracer activities described in Tables A1-A3 (see Appendix) are recommended for an average $70-\mathrm{kg}$ patient imaged in an Anger camera. In addition, injected activity ranges for patients with a body mass index (BMI) greater than or equal to $35 \mathrm{~kg} / \mathrm{m}^{2}$ are included in the tables. For 1 day rest and stress studies, typically, the first study (rest or stress) is the lower dose study, while the second study (stress or rest) is the higher-dose study. 
When performing two-day studies, the stress study should be performed first, if possible, because if these images are normal, the rest images are not needed, thus reducing the patient radiation exposure. Dose-reduction techniques by increasing the acquisition time or by using resolution recovery/noise reduction software solutions ${ }^{41}$ or new collimator designs are recommended. ${ }^{42}$ For CZT cameras, standard doses are listed in Tables A4-A6 (see Appendix); the greater sensitivity of the system allows tailoring of protocols to minimize radiation exposure (e.g., for younger patients) or minimize acquisition time (e.g., for patients who cannot remain motionless for longer acquisition times). With some of these systems, however, one may acquire images to reach a specific number (such as 1 million) of cardiac counts, which can greatly reduce the acquisition time in the majority of patients. ${ }^{43}$ Radiotracer activity adjusted to patient body habitus, imaging protocol, SPECT instrumentation, and type of radiotracer is recommended.

\section{Position}

Factors influencing patient position include camera or gantry design (as discussed in section "Patient configuration"), reduction of artifacts, and patient comfort. Supine position is routinely used for SPECT imaging. Prone imaging has been reported to produce less patient motion and less inferior wall attenuation than supine imaging. ${ }^{44,45}$ The combination of supine and prone images (2-position imaging) may be helpful identifying attenuation artifacts due to the shift in position of the attenuating structures that occur in the prone position and can improve interpretive confidence and accuracy. ${ }^{46-48}$ By comparing supine and prone images, artifactual defects will resolve or change their location whereas true perfusion defects will remain in the same position. ${ }^{19,49}$ When being used in this fashion, the acquisition time for the secondary (prone) image set can be reduced by $20 \%$ to $40 \%$.

With the two available upright SPECT systems, routine acquisition both the supine and upright positions (2-position imaging) is recommended. Image interpretation follows a similar scheme to the supine and prone imaging previously discussed. True perfusion defects are present in the same location on supine and upright images, however, the left ventricular (LV) itself may rotate slightly. ${ }^{20,21}$ The importance of acquiring at least post-stress images in both positions was underscored by the finding that upright imaging reclassified $62 \%$ of equivocal images as normal and $11 \%$ as abnormal. ${ }^{50}$

Changes in patient positioning from those described previously will likely cause changes in the distribution of adjacent soft-tissue attenuation and need to be considered in image interpretation. Position-specific normal databases will most likely improve image quantification. Care must be taken in positioning patients within cardiocentric CZT cameras and multifocal collimator Anger cameras, which have smaller FOV and focus the detectors on the heart volume. Improper positioning of the heart within the quality FOV of the camera can affect the severity of defects and cause truncation artifacts. In addition, differences in positioning between stress and rest acquisitions may cause artifactual defects with variable degrees of reversibility. ${ }^{12,51}$ Proper positioning of the patient so the heart is in the center of the quality FOV can be accomplished based on real-time persistence imaging. For individuals with dextrocardia, feet-first position on Anger camera can be used to acquire images keeping all other parameters the same.

In summary, supine position is standard for SPECT MPI with most currently available Anger and CZT systems; the routine use of 2-position imaging, combination of supine followed by prone acquisition or upright and supine imaging, is recommended, at least for stress images, particularly if AC is not available.

\section{Delay Time for Imaging}

The delay times from injection of radiotracer to start of SPECT scan are listed as ranges. The objectives are to allow clearance of subdiaphragmatic activity, and to allow the patient to recover fully from exercise, thus allowing the heart rate to return to baseline (reducing gating artifact), avoiding "upward creep" from changes in respiratory patterns while dyspnea resolves and to minimize interference from hepatic uptake. ${ }^{52}$ Provided that imaging times fall within the specified ranges, clinically useful SPECT images should result. With ${ }^{201} \mathrm{Tl}$, imaging should begin approximately 10 to 15 minutes after stress testing. Care should be taken to optimize initial imaging and to avoid repeat imaging with ${ }^{201} \mathrm{Tl}$, as significant ${ }^{201} \mathrm{Tl}$ redistribution has been observed as early as 20 minutes after flow restoration, which may reduce the extent and/or severity of true defect(s). In contrast, the properties of ${ }^{99 \mathrm{~m}} \mathrm{Tc}$ sestamibi and ${ }^{99 \mathrm{~m}} \mathrm{Tc}$ tetrofosmin, particularly the lack of clinically significant redistribution or washout, allow delayed imaging and, therefore, permit stress testing and tracer injection to take place at a location remote from the imaging laboratory. Image acquisition can simply be repeated when patient motion or extracardiac tracer uptake is considered responsible for the production of a perfusion defect. The standard delay between injection of ${ }^{99 \mathrm{~m}} \mathrm{Tc}$ sestamibi or tetrofosmin and scan is 30 to 60 minutes for rest and 15 to 60 minutes for stress (the former for exercise stress). 


\section{Energy Windows}

Energy window position for image acquisition is determined by the radioisotope employed, $140 \mathrm{keV}$ for technetium-based perfusion agents and $70 \mathrm{keV}$ for ${ }^{201} \mathrm{Tl}$. It is reasonable to acquire the higher energy peaks of ${ }^{201} \mathrm{Tl}(135$ and $167 \mathrm{keV})$ simultaneously on cameras capable of doing this. The window sizes are determined largely by the imaging systems (i.e., detector material) and most often reflect the tradeoff between image counts and resolution. The CZT systems offer improved energy resolution, and therefore the energy window width may be reduced to decrease scatter and improve image resolution. Furthermore, the greater energy resolution of the CZT camera may allow simultaneous dual isotope imaging of ${ }^{99 \mathrm{~m}} \mathrm{Tc}$-based and ${ }^{201} \mathrm{Tl}$ perfusion tracers ${ }^{53}$ or molecular imaging agents, such as ${ }^{123}$ I- $\beta$-methyl-iodophenyl-pentadecanoic acid (BMIPP) ${ }^{54}$ It is important to remember that the same energy windows used in performing patient studies should be used for routine, daily QC. Some of the scanners acquire dual energy windows $\left(120 \mathrm{keV}\right.$ for $\left.{ }^{99 \mathrm{~m}} \mathrm{Tc}\right)$ to allow for scatter correction.

\section{Collimator}

For conventional Anger imaging systems, parallelhole collimators are most commonly employed for cardiac SPECT acquisitions and are discussed in detail in "Collimators" section.

\section{Angular Sampling Range}

Because of the anterior position of the heart in the left hemithorax, the standard orbit or angular sample range is $180^{\circ}$ from $45^{\circ} \mathrm{RAO}$ to $45^{\circ} \mathrm{LPO}$. The recommended orbit range is largely dependent on the camera configuration, and the choice should consider the manufacturer's recommendation. The array of detectors with the currently available solid-state CZT cameras effectively covers a similar angular sample range as the conventional Anger cameras.

\section{Number of Projections}

The optimal number of projections for emission studies depends on matching the number of projections to the resolution of the system. A ${ }^{201} \mathrm{Tl}$ SPECT acquisition with a low-energy all-purpose (LEAP) collimator is a relatively low-resolution study, for which 32 projections acquired over $180^{\circ}$ is sufficient. A higher resolution study using ${ }^{99 \mathrm{~m}} \mathrm{Tc}$ agents should be collected with a high-resolution collimator; this requires at least
60 to 64 projections over $180^{\circ}$ to prevent loss of resolution.

The two commercially available CZT systems utilize differing strategies for image acquisition and therefore acquire differing numbers of projections. The 9 detectors in the D-SPECT system (Spectrum Dynamics) simultaneously rotate through a small angle to optimize coverage of the cardiac volume. In doing this, 120 projections are acquired. In contrast, the 19 detectors of the Discovery 530c (General Electric) system remain in a fixed position focused on the cardiac volume, therefore generating 19 projections.

\section{Orbit Type}

For Anger cameras with rotating detector systems, the main orbit options for SPECT cardiac imaging are circular and noncircular (elliptical or body-contoured) orbits. Noncircular orbits follow the contour of the patient closer than circular orbits with fixed radius, thereby improving spatial resolution. In general, there is reduced (but more uniform) spatial resolution with circular orbits because the detector-to-source distance is greater with this technique. Manufacturers are more commonly providing excellent noncircular orbit capability with the ability to minimize the negative impact of variation of source-to-detector distance. Circular and noncircular (elliptical or body-contoured) orbits are standard for Anger camera SPECT MPI.

\section{Pixel and Matrix Size}

For most conventional Anger camera systems, the effective imaging resolution is between 13 and $16 \mathrm{~mm}$. For spatial sampling, it is desirable to have two to three pixels over the imaging resolution, which provides a usable pixel range of $4.5 \mathrm{~mm}$ to $7.0 \mathrm{~mm}$ per pixel. Tables A1-A3 (see Appendix) specify a $6.4 \pm 0.4 \mathrm{~mm}$ pixel size for a $64 \times 64$ image matrix, which has been extensively used with current Anger technology. This size offers satisfactory image resolution for interpretation and quantitation of both ${ }^{201} \mathrm{Tl}$ and ${ }^{99 \mathrm{~m}} \mathrm{Tc}$ tomograms with standard reconstruction methods. However, recent studies have demonstrated the potential superiority of 128 matrix with 3.2-mm pixel size when new reconstruction methods with resolution recovery are used on standard SPECT cameras. ${ }^{55}$ For the CZT SPECT camera systems, the usual pixel size is $2.5 \mathrm{~mm}$ and $128 \times 128$ matrix size.

\section{Acquisition Type}

The most widespread mode of tomographic acquisition for the conventional Anger cameras is the "stepand-shoot" method. In this approach, the camera 
acquires a projection, then stops recording data when moving to the next angle; this results in a small amount of dead time because the camera is not acquiring data while it is moving. An alternative is "continuous" mode where the camera moves continuously and acquires each projection over an angular increment. This eliminates dead time and thus increases image counts at the expense of a small amount of blurring due to the motion of the camera head while acquiring. It seems likely that the increase in count statistics more than offsets the small amount of blurring due to camera motion. As noted previously, the detectors in the solid-state CZT imaging systems are fixed in position around the left hemithorax, and therefore a "step-and-shoot" acquisition method is not feasible. Similarly, a "continuous"acquisition mode in the sense employed by Anger cameras also does not occur, although the CZT detectors are continuously acquiring during the imaging protocol. List-mode dynamic acquisition of data using the CZT systems can be used for determination of MFR.

\section{Acquisition Time}

The total time for an emission acquisition often is based on the need to acquire sufficient counts balanced by how long a patient can tolerate the procedure without moving. The maximum practical time is on the order of less than or equal to 20 minutes for conventional Anger cameras. Because of the 5- to 6-fold higher count sensitivity of the CZT imaging systems compared with the Anger cameras, the typical acquisition time for these systems is between 2 and 14 minutes, depending on the radiotracer activity. However, a more standard 10- to 15-minute acquisition could be used with very low injected activity. The acquisition times provided in Appendix Tables A1-A6 have been found to produce images of acceptable and comparable quality for rest and stress studies. These times should be adjusted based on the manufacturer's recommendation.

\section{Gating}

The introduction of technetium-based perfusion tracers has resulted in images with sufficient count density to allow for cardiac gating adding parameters of wall motion, wall thickening, and ejection fraction (EF) to MPI. ${ }^{56-59}$ Gated acquisition should be performed whenever technically feasible. Gating requires a stable and consistent heart rhythm, as well as sufficient temporal resolution to characterize the cardiac cycle correctly. A stable heart rate and rhythm can be achieved by rejecting heartbeats that fall out of range at the expense of an increase in image time. This "beat-length acceptance window" can vary from $20 \%$ to $100 \%$ of the expected R-R duration; the recommended value being $20 \%$ if an "extra frame", is provided, which allows the accumulation of rejected counts. Most laboratories gate the heart for 8- or 16frames per cycle. The increased temporal sampling has been used to derive more accurate estimates of ejection fraction (EF) as well as parameters of diastolic function. ${ }^{60,61}$ It is important to review the $\mathrm{R}-\mathrm{R}$ interval histogram for the study acquisition to determine if there is a high degree of variability that may affect measurements of LVEF. For either 8- or 16-frame gating, the recommendations are to avoid beat rejection for ungated perfusion reconstruction by using the "extra frame", to collect perfusion data not included in the 8or 16-frame gating. The lower count statistics achieved with ${ }^{201} \mathrm{Tl}$ imaging makes gating more challenging with this isotope, but many laboratories have reported satisfactory results using 8-frame gating in selected patients. ${ }^{62}$ Gated-SPECT MPI with minimum of 8frame gating is standard. Whenever feasible (assuming adequate count density of the low-dose scans), both stress and rest SPECT perfusion studies should be acquired as gated data sets to aid in the evaluation of transient LV dysfunction following stress.

\section{Attenuation Correction}

Correction of soft-tissue attenuation, AC, can improve the specificity and normalcy rate of visual and quantitative analysis of cardiac SPECT MPI. ${ }^{63-65}$ Shallow tidal breathing and a tube current of 10 to 20 $\mathrm{mA}$ and a tube voltage of 80 to $140 \mathrm{kVp}$, scan duration 10 to 30 seconds are traditionally used but lower tube currents are being used with slow rotation CT systems and may become the standard in the future. For radionuclide $\mathrm{TCT}$, the recommended parameters are shallow tidal breathing and a duration of the scan 3 to 5 minutes. Longer duration is recommended for older radionuclide sources. Also see "Attenuation Correction'.

\section{Recommendations for SPECT MPI Acquisition}

- ASNC recommends acquisition of MPI in a gated mode with parameters tailored to the individual patient, the scanner, and the clinical question.

- The lowest possible radiotracer activity for the camera system without jeopardizing image quality, adjustment of imaging time based on radiotracer activity, use of the maximum pixel count possible with the camera, use of AC or 2-position imaging, and use of stress-first imaging when feasible are recommended. 


\section{PROCESSING}

\section{Filtering}

SPECT quality is improved by means of image enhancement via noise reduction. ${ }^{66,67}$ All forms of imaging are plagued by statistical variation in the acquired image counts commonly referred to as noise. The quality of an image can be described by the signalto-noise ratio, which describes the relative strength of the signal component (the object that is imaged) compared to noise. The signal to-noise-ratio is much higher at lower spatial frequencies (broad features that are constant over many pixels) and decreases at higher spatial frequencies (features that change over few pixels, such as edges). In general, the greater the count statistics, the better is the signal-to-noise ratio. A lowpass filter is generally used to reduce noise because it allows low spatial frequencies to pass through and attenuates the high frequencies where image noise predominates. Low-pass filters such as the Hanning and Butterworth can be characterized by a cut-off frequency where they begin to affect the image. The cutoff frequency can be adjusted, depending on the signalto-noise ratio to preserve as much of the signal and suppress ${ }^{68}$ as much noise as possible. If the cut-off is too high, there is significant noise in the image; if the cut-off is too low, significant information in the signal is suppressed so the image becomes too smooth. Nuclear cardiology images, because of their relatively low count statistics, tend to have greater amounts of image noise and filtered backprojection (FBP), because of its dependence on ramp filtering, tends to amplify this noise. The optimal filter for a given image depends on the signal-to-noise ratio for that image; underfiltering an image leaves significant noise in the image, and over-filtering unnecessarily blurs image detail; both over filtering and under filtering can reduce image accuracy. Software reconstruction packages are set with default filter selection and cut-off values that are optimized for the average patient. Adjustment of the filter cut-off can be done in patients with poor count statistics (e.g., obese patients) to optimally filter their images (Figure 8, example of varying image quality depending on cut-off frequency). This is discouraged, however, unless the physician is thoroughly familiar with filter adjustment and the potential effects. Moreover, changing the filter cut off may have unexpected effects on the output of commercially available analysis programs, especially those that employ edge detection such as defect quantitation and LV volumes and EF.

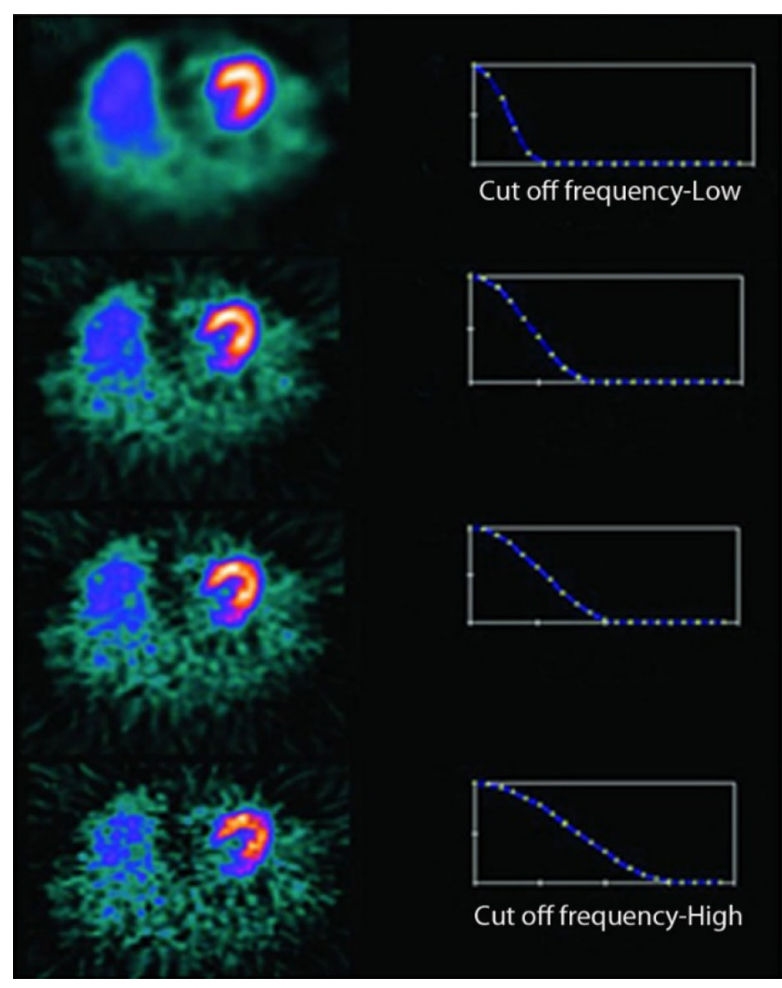

Figure 8. Example of varying image quality depending on cut-off frequency.

\section{Reconstruction}

Filtered backprojection. The traditional method of image reconstruction has been FBP, a technique based on a mathematical proof, which assumes perfect line integral count profiles (i.e., perfect collimation), no attenuation, no scatter, and an infinite number of projections. It is relatively straightforward and comparatively fast. ${ }^{69}$ Simple backprojection of acquired count profiles (a single row of pixels from each projection) results in an image that appears blurred by a function of $1 / r$ where ' $r$ ' equals the radial distance in the Fourier domain. To suppress this blurring, each profile is filtered in the Fourier domain with the ramp filter prior to backprojection. As noted previously, application of the ramp filter, although suppressing low frequency $1 / r$ blurring, amplifies the already relatively noisy high-frequency content of the acquired profiles. Also, in the presence of subdiaphragmatic tracer concentration (i.e., stomach, liver, or bowel) in the $x$-plane of the heart, a ramp-filter artifact is frequently introduced where counts are subtracted from the inferior wall of the heart in the $x$-plane of the subdiaphragmatic activity (see Figure 9, ramp filter schematic and patient example). Therefore, it is a combination of the ramp 


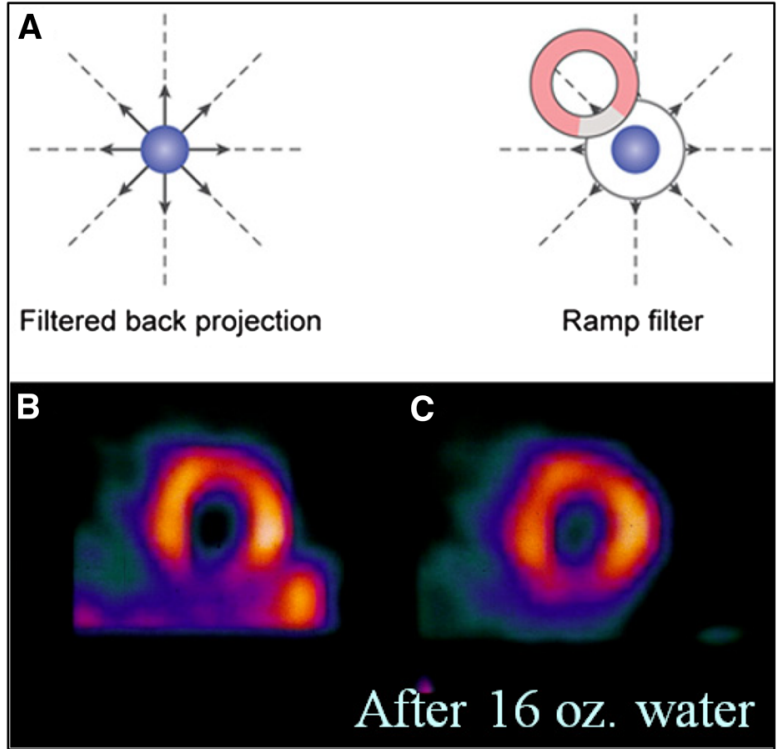

Figure 9. (A) Ramp filter artifact schematic, (B) effects on myocardial perfusion images, and $(\mathbf{C})$ improved after water intake. The pink circle represents short axis view of the LV. The grey sector represents count reduction in the inferior wall from ramp filter artifact.

filter and a low-pass, noise-suppression filter (e.g., Butterworth) that is ultimately used to filter the projection data prior to backprojection.

Iterative reconstruction. There is a different class of reconstruction algorithms that is based on iterative techniques. These algorithms start with a rudimentary guess of the radiotracer distribution, generate projections from the guess, and compare these projections to the acquired projections. The guess is refined based on the differences between the generated and actual projections, and the process is repeated (hence the term 'iterative') usually for a fixed number of iterations but can also be repeated until the error between the generated and actual projections is acceptably small. A main advantage of these algorithms is that the process of generating projections from the guess can be made as sophisticated as desired and can incorporate corrections for attenuation, scatter, and collimator specific, depth-dependent blurring (see below). Various iterative algorithms have been developed including, but not limited to, expectation maximization (EM), maximum likelihood expectation maximization (MLEM), and maximum a posteriori (MAP) techniques. The primary differences between these algorithms are the methods by which re-projected data (the guess) is compared and updated relative to the acquired projections and differences in noise modeling and compensation. In an effort to decrease reconstruction time, the ordered subsets expectation maximization (OSEM) algorithm was developed and is now almost universally used for iterative reconstruction of SPECT by all vendors. ${ }^{70}$ This technique uses a subset of the acquired projection data during each iteration with the number of subsets speeding the reconstruction time by roughly the same factor-i.e., a reconstruction using four iterations with eight subsets completes roughly eight times faster than a non-subsetted EM reconstruction. ${ }^{71}$ This technique, coupled with the increased speed and power of commercially available nuclear medicine computers, allow iterative reconstructions to be completed in an acceptable time for routine clinical use. The reconstruction methods discussed thus far generally reconstruct, in isolation, one transaxial two-dimensional (2D) slice per projection count profile-one row of pixels from each projection reconstructs and creates a single slice, each derived independently from the previous or next. Newer iterative techniques use data from adjacent count profiles and 2D slices to better model data acquisition and 3D tracer distribution. Therefore, because of all the advantages described previously, iterative reconstruction is currently preferred to FBP for reconstruction of gated and ungated myocardial perfusion SPECT images. Typically, mild 3D post-filtering is additionally applied after the reconstruction process.

Reduced count density reconstructive techniques. New SPECT reduced count density reconstructive techniques have been introduced to improve SPECT image quality despite lower count statistics. ${ }^{72}$ Consequently, SPECT acquisition time may be shortened. Alternately, the injected radiotracer activity may be decreased, thereby decreasing patient radiation exposure. These methods, described below, are all now commercially available. They are available on all new cardiac cameras and may also be available for reconstruction of data from conventional Anger camera systems.

Iterative reconstruction, described previously is used for all reduced count density reconstruction techniques. Specifically, 3D reconstruction methods are employed. For example, instead of modeling collimator point-spread functions simply in the 2D-transaxial plane (fan beam), collimator depth-dependent deblurring can be applied incorporating data in the axial direction as well (cone beam), across multiple 2D slices (i.e., 3D reconstruction). Most use an OSEM algorithm, which can be broadly characterized as a 3D-OSEM technique. These techniques can compensate for the increased noise of low-count acquisitions, thus allowing for a decrease in acquisition time (half-time or quarter-time imaging has been investigated $)^{73-75}$ or a reduction in injected activity, and therefore, decreased patient radiation exposure. 
Resolution recovery. Reduced count density reconstructive techniques also incorporate collimatordetector response modeling, more commonly referred to as "resolution recovery." The high-resolution, parallelhole collimator used for myocardial perfusion SPECT maintains spatial resolution by accepting photons emitted from the myocardium, traveling directly perpendicular to the face of the camera and the parallel holes of the collimator. Photons emanating from voxels not directly perpendicular to the collimator hole are attenuated by the collimator's lead septa. Photons emanating from these voxels may undergo Compton scattering and may also subsequently travel perpendicular to the camera hole. These are eliminated by the pulse-height analyzer, which rejects photons losing more than $10 \%$ to $15 \%$ of their initial photon energy. However, these advantages of the parallel-hole collimator are progressively compromised the more distant the voxel is from the camera face (Figure 10, graphic distant-dependent blurring). The greater the distance of object from the camera face, the greater the likelihood that photons from adjacent voxels will pass through the parallel-hole collimator. Likewise, the likelihood that Compton-scattered photons will be accepted progressively increases. For these reasons, spatial resolution of a camera decreases with object distance from the camera face. Therefore, it has always been emphasized that when a patient is positioned for myocardial perfusion SPECT, the face of the scintillation detector should be as close as possible to the patient's chest wall. Likewise, a non-circular orbit is preferable because it allows the camera head to more closely approximate the chest wall in all projections. The magnitude of this loss of resolution is directly proportional to the width of the collimator hole and inversely proportional to the hole length (Figure 11, graphic loss of resolution with increasing collimator hole width). Septal penetration of photons is another cause of decreased resolution of a parallel-hole collimator. However, for the relatively low

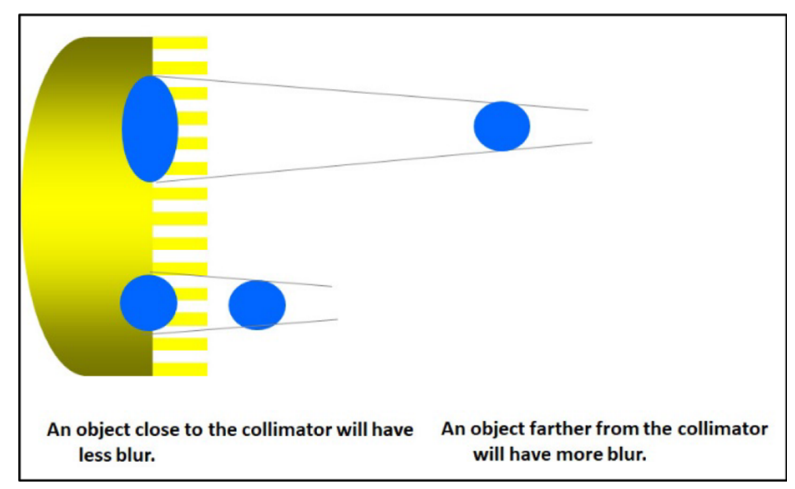

Figure 10. Distance-dependent blurring.

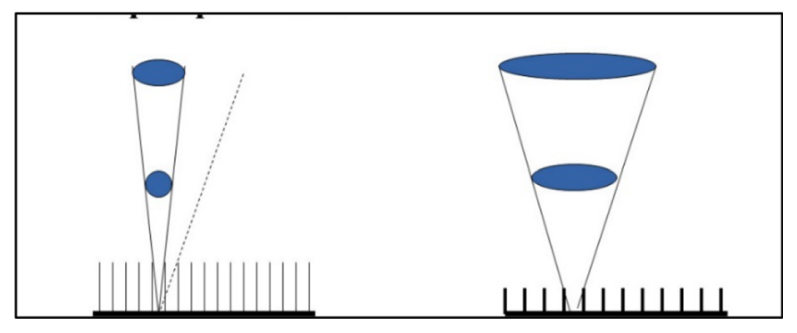

Figure 11. Collimator parameters that influence depth-dependent loss of spatial resolution. Increasing collimator hole width, decreasing hole length, and increasing septal penetration result in loss of resolution. ${ }^{72}$

energy isotopes, ${ }^{99 \mathrm{~m}} \mathrm{Tc}$ and ${ }^{201} \mathrm{Tl}$, the degree of septal penetration is minimal.

This distance-dependent collimator-detector blur, dependent upon the shape of the holes, their dimensions, and the thickness of the septa for each individual collimator has a major influence on image resolution. ${ }^{76,77}$ It is the main factor affecting the resolution and noise properties of nuclear medicine images. In reconstructed SPECT images, these effects are strongly influenced by the applied reconstruction algorithm and its parameters. Resolution recovery models the physics and geometry of the emission and detection processes. It is thereby a means to compensate for the collimator-detector response $(\mathrm{CDR})$ in iterative reconstruction. ${ }^{78-80}$ The CDR consists of four main components: intrinsic response (the system without a collimator) and the geometric, septal penetration, and septal scatter components of the collimator parameters. By including an accurate model of CDR function in an iterative SPECT reconstruction algorithm, compensation for the blurring effect may be included in the iterative reconstruction process, resulting in improved spatial resolution and a reduction in image noise. For each combination of acquisition system, radiotracer, and particular-acquisition protocol, the CDR function provides the probability that a photon emitted from any point of the imaged object will contribute to a pixel of the resulting image. Accurate predictions of the geometric response function for various collimator designs have been derived. This is accomplished by knowing the CDR for each particular scintillation camera/collimator system.

To implement resolution-recovery acquisition parameters, the COR and the collimator-to-voxel distances for every acquired projection must be known. Additionally, to apply pixel weighting appropriately to the image matrix, which includes the heart and surrounding body structures, the distance from the detector to the patient's body must be determined. Newer cameras automatically provide this distance in the acquired parameters for each angular position. Typically, 


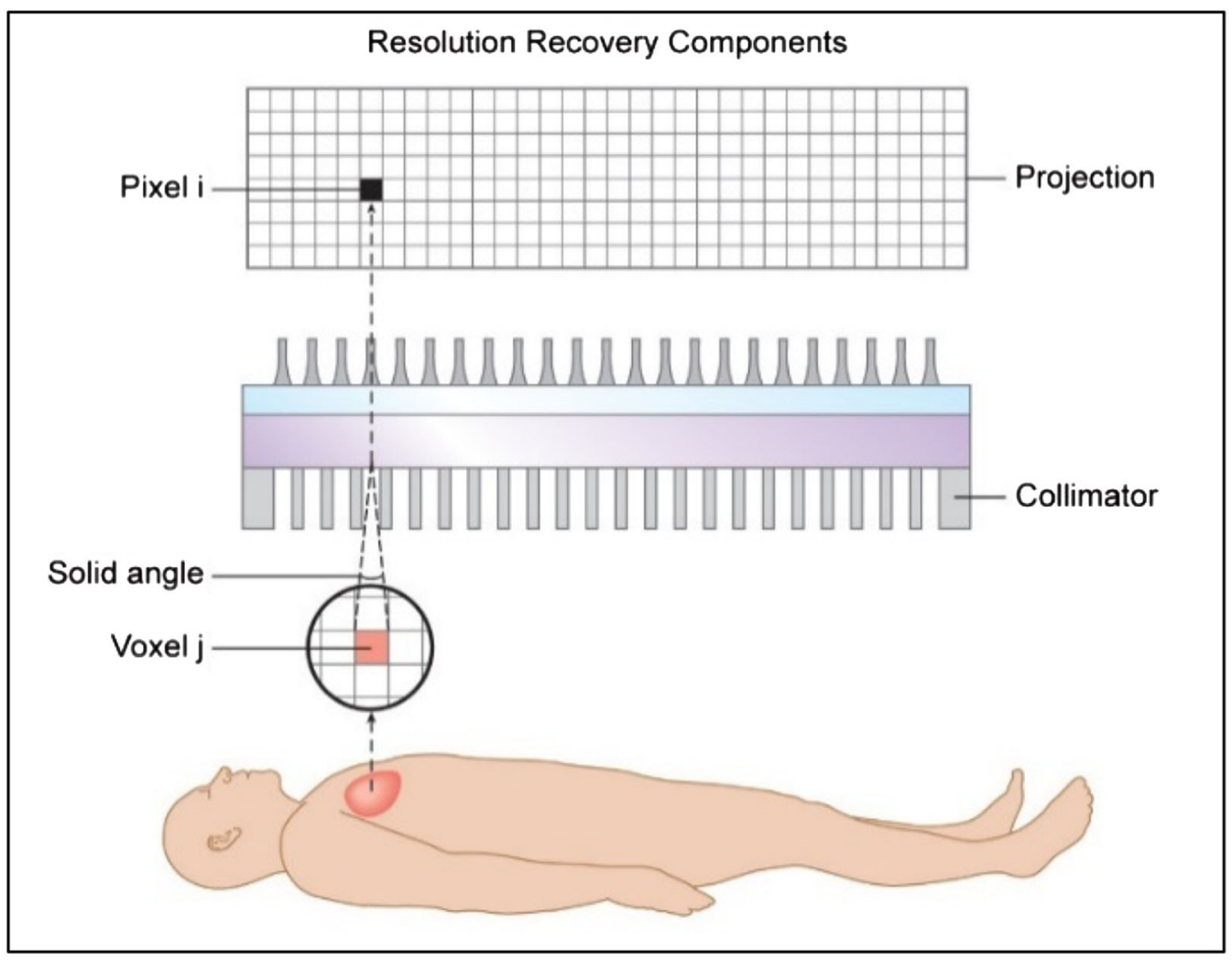

Figure 12. Resolution recovery.

resolution recovery models the $\mathrm{CDR}$ and the physics and geometry of the emission and detection processes. ${ }^{73}$ As previously described, the shape of the holes, their dimensions, and the thickness of the septa for each individual collimator have a major influence on image resolution. Therefore, during iterative reconstruction, data are modified in each reconstructed voxel according to the collimator's geometry. Pixel weights are calculated analytically, taking into account the solid angles subtended by the collimator between each detector pixel and each body voxel (Figure 12). Thus, resolution recovery yields images of improved spatial resolution and with less noise as compared to conventional techniques. ${ }^{73}$ Clinical images of lower count density acquired with resolution recovery have been demonstrated to be equivalent or superior to those acquired with conventional SPECT imaging, allowing for reduced SPECT acquisition time or reduced injected radiotracer dose.

Noise compensation/reduction. Noise compensation/reduction methods are also incorporated into reduced count density reconstructive techniques. Their precise implementations vary according to vendor. Nuclear imaging data are inherently noisy due to relatively poor counting statistics. Low-count density myocardial perfusion SPECT images are characterized by noise, which has similar or higher magnitude compared to the high-frequency portion of the true myocardial data. As described previously, FBP eliminates high-frequency data, thereby "smoothing", (i.e., blurring) the image. Such filtering results in decreased image contrast, decreased spatial resolution, and potentially decreased diagnostic sensitivity in detecting perfusion and regional wall motion abnormalities. In contrast, with noise compensation methods, signal-tonoise values are determined by the resolution and smoothness desired in the final cardiac SPECT image. High-frequency components of the image are suppressed while loss of resolution is minimized.

Typically, noise compensation/reduction enhances the signal-to-noise ratio (SNR) by modeling the statistical characteristics of the emission process and of the detected data. ${ }^{74}$ High-frequency components present in the projections are suppressed. The balance between noise and myocardial signal is determined adaptively and automatically according to the data analysis and desired smoothness. The smoothness of the image is guided by the application's target SNR. ${ }^{81}$ The 


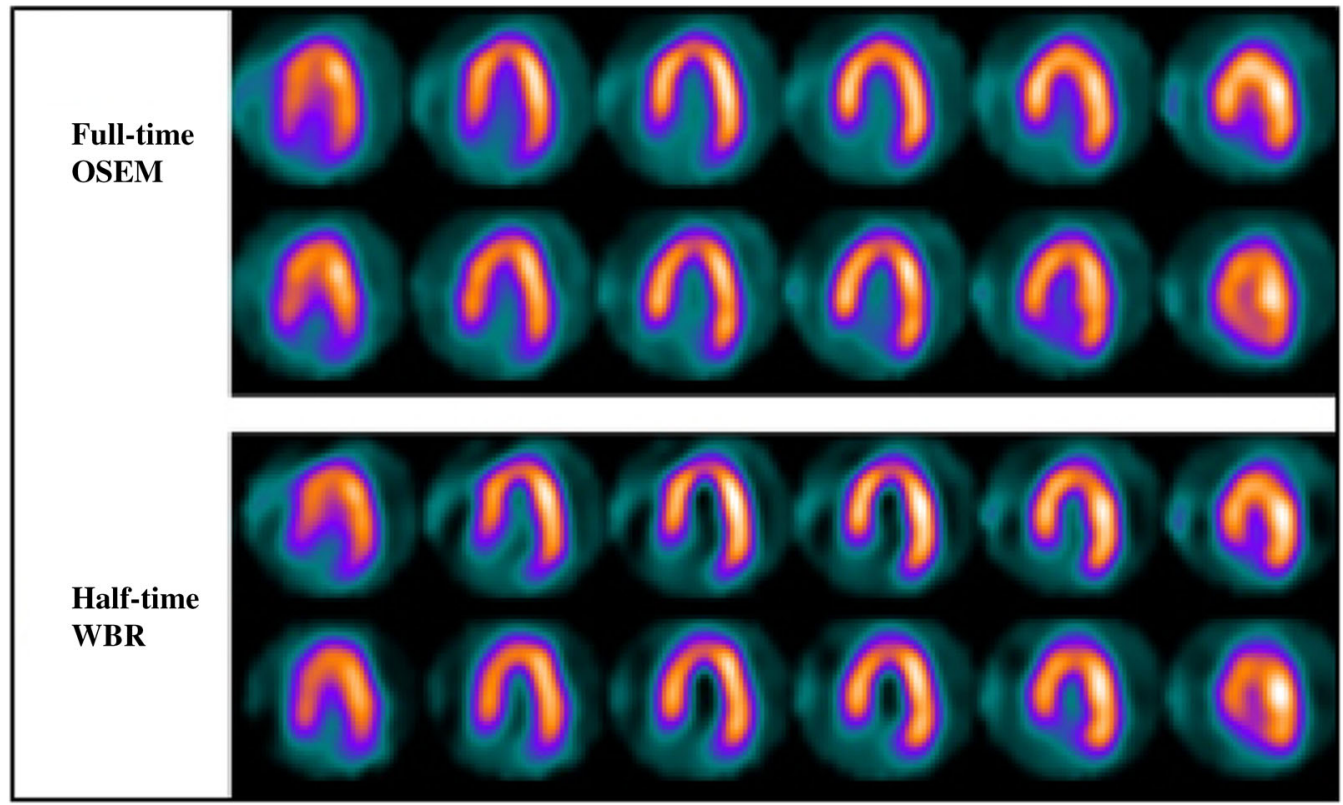

Figure 13. Case example.

combination of iterative reconstruction, resolution recovery, and noise compensation/reduction preserve or improve image quality in reduced-count density images, thereby allowing for reduced injected activity, reduced SPECT acquisition time, or both (see Figure 13).

Pre-determined SPECT acquisition times. The introduction of dual-head cameras more than two decades ago allowed us to halve acquisition times as compared to acquisition times for studies performed on older single-head cameras. Since their introduction, however, cardiac SPECT protocols have been rather rigid with regards to image acquisition times. For larger patients with soft-tissue attenuation resulting in lowcount density studies, "weight-based dosing" was recommended to increase cardiac SPECT counting statistics. However, by increasing the radiotracer dose, patient radiation exposure is likewise increased. As an alternative to increasing the radiotracer dose, it is possible to increase SPECT acquisition time in cooperative patients. Newer "smart" cameras allow optimal SPECT acquisition times to be predetermined in such patients. The camera operator can accept or reject this suggested acquisition time, depending on the patient's tolerance. By this means, optimal counting statistics can be obtained in larger patients without increasing the radiotracer dose.

\section{Attenuation Correction}

Attenuation correction requires an estimate of the patient tissue densities. Estimates may be as simple as a patient body outline, or patient specific via the use of transmission image acquired with either radioisotope sources or computed tomography (CT) scans. Attenuation correction with FBP is not accurate in the thorax and is not recommended. Attenuation correction should only be applied in conjunction with an iterative reconstruction algorithm. With iterative reconstruction, the effects of attenuation are included in the reconstruction as part of the system model. ${ }^{82}$

Attenuation. Gamma rays emitted from within the myocardium can be lost to attenuation before leaving the patient and being detected by the gamma camera. For $140-\mathrm{keV}$ gamma rays of ${ }^{99 \mathrm{~m}} \mathrm{Tc}$, approximately $12 \%$ of gamma-rays will be attenuated for every centimeter of soft tissue that is passed through. One can correct for this attenuation by determining the total attenuation along a line from where the gamma ray was emitted to the edge of the patient. In the thorax, the attenuating properties of the various tissue and organs differ, and so a distribution of the attenuating materials is required. This attenuation map is derived from a transmission image, which can be acquired from a radionuclide source, such as Gd-153 (gamma-rays with energies of 97 and $103 \mathrm{keV}$ ) transmission scan or an X-ray CT scan. In both cases, the values within the attenuation map are specific to the energy of the radionuclide that is being used for the SPECT scan. For transmission scans, the attenuation map is measured for the energy of the transmission source and then scaled to the emission radionuclide. For CT, the voxel values within the CT image, which have units of $\mathrm{HU}$, are converted to the 


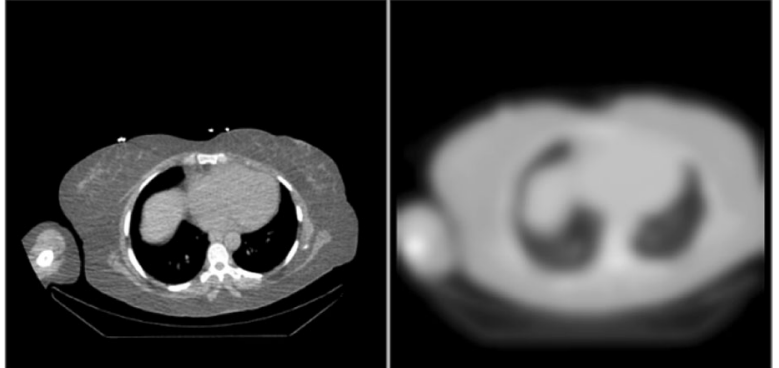

Figure 14. A CT cross-section (left) and corresponding attenuation map (right). Note the attenuation map appears larger as the same zoom factor applied to the SPECT scan has been applied.

attenuation coefficients for the emission radionuclide (see below). Once this attenuation map is created, it is utilized within the forward and back-projection steps of the iterative reconstruction process.

Optimization of CT for AC. The voxel dimensions of the attenuation map are required to match those of the SPECT image, which are usually between 3 and $7 \mathrm{~mm}$. This is considerably larger than the voxel dimensions of the CT images that will usually be acquired on $512 \times 512$ matrix and have sub-millimeter voxels. Consequently, the CT image matrix is downsampled to match that of the emission matrix. In addition, a smoothing filter is applied to the attenuation map in order to degrade the spatial resolution to be comparable with the SPECT image. The combination of the downsampling and filtering results in an attenuation map that is very low noise. This is illustrated in Figure 14

This noise reduction that occurs during the conversion of the CT to the attenuation map means the process is very robust, even with noisy CT data. Therefore, the CT parameters should be set to deliver a very low-dose CT. If it is possible, the CT-slice thickness should be set to be roughly comparable to the pixel size of the emission data. Thin CT slices offer no advantage when converting to the attenuation map due to the matrix downsampling.

Estimating the radiation effective dose from the CT exposure is challenging due to uncertainty in how effective dose varies for different patient body habitus. As such, when considering optimization of the CT exposure, it is more appropriate to focus on a measure that is obtained directly from the CT acquisition system. The most common metric is the dose length product (DLP), which is the product of the normalized dose-perunit length and the total axial length of the scan and taking units of $\mathrm{mGy} \mathrm{cm}$. For older hybrid SPECT/CT systems, such as the Hawkeye system (from General Electric Healthcare), the DLP should ideally be less than $40 \mathrm{mGy} \mathrm{cm}$. On modern systems, the DLP should ideally be less than $20 \mathrm{mGy} \mathrm{cm}$. Dose modulation may be used to provide more consistent image quality in larger patients. However, care should be taken when defining the particular image quality metric to ensure it is suitable for the entire patient population, keeping the dose as low as possible. For departments that use CT for $\mathrm{AC}$ on a routine basis, periodic audits of DLP should be performed to ensure continued adherence to defined DLP levels. ${ }^{83}$

Practical considerations. Axial coverage of CT. For operators of hybrid SPECT/CT equipment, it is essential when using CT (with its resultant patient radiation exposure) that attenuation-corrected images of diagnostic value are acquired. There are several practical steps that all operators should follow to make best use of the CT while optimizing the radiation exposure to the patient. A key consideration affecting the $\mathrm{CT}$ radiation dose is the axial coverage of the CT scan. It is important to judge sufficient CT axial coverage to include all the myocardium but also allow sufficient margin superior and inferior without irradiating excessive sections of the patient. This margin is to account for some motion due to respiration and to allow manipulation of the attenuation-corrected SPECT image when reorienting for the long- and short-axis views. If the limits of the axial coverage are too close to the myocardium then there is a risk of truncation in the axial direction. Alternatively, the entire myocardium may be covered by the CT, but truncation artifacts can occur when the myocardial image volume is manipulated. In both circumstances, the attenuation-corrected images are not of diagnostic quality, and the patient will have received an unnecessary radiation exposure.

It is important to use the emission data to guide the axial coverage of the CT scan. This can be performed on the persistence view before the SPECT is started. This can be challenging due to the noisy quality of the persistence image and reinforces the need to apply a margin, as shown in Figure 15. Alternatively, it may be possible to define the CT limits from reconstructed SPECT images, which will offer superior visualization of the myocardium. This latter method should be used preferentially if it is available.

Image checks prior to CT scan. The SPECT scan should always be performed before the CT scan. This will allow the operator to check the quality of the SPECT data just after it has been acquired to ensure it is diagnostic quality. If the initial SPECT is non-diagnostic, then applying $\mathrm{AC}$ will certainly not produce diagnostic images, and the patient will go on to receive an unnecessary radiation exposure. This is an essential 

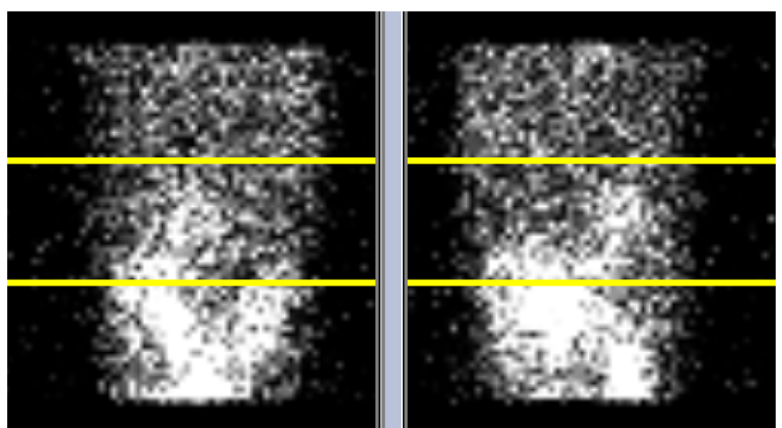

Figure 15. Persistence view used to define the axial coverage-defined by the yellow lines-of CT for attenuation correction as viewed on an Infinia Hawkeye system (GE Healthcare).

check that must be performed before the $\mathrm{CT}$ is started. It should easily take less than one minute if the work area is set up properly. It can usually be achieved as the camera is moving from the SPECT to the CT position so minimal delay occurs. Key things for the operator to identify are excessive patient motion or high levels of extra-cardiac activity. The former can be identified by reviewing the cine display and sinogram, while the latter should be preferably viewed on the reconstructed images without attenuation correction. On some systems, this will be possible on the acquisition workstation, while other systems may require a processing workstation to allow it. Therefore, consideration should be given to the operator's control area to ensure all image checks can be performed.

Pitfalls. There are two main pitfalls in performing attenuation correction. The first of which is truncation of the attenuation map. The second is misregistration between the emission data and the transmission data.

Truncation. Truncation of the attenuation map will result in the incorrect path length, and resultant calculation of attenuation, from the gamma-ray emission to the outside of the patient. Attenuation maps generated from both radionuclide transmission scans and CT scans are both susceptible to truncation.

Small FOV-dedicated cardiac SPECT systems that use radionuclide transmission sources are particularly susceptible to truncation due to the size of the FOV. While vendors offer algorithms to reduce truncation, it is possible for artifact to still occur in certain patients as illustrated in Figure 16.

Truncation of a CT-derived attenuation map can occur from two factors. The first is truncation of the CT data itself. A CT scan will usually have a transaxial FOV diameter of $50 \mathrm{~cm}$, therefore, it is possible for certain portions of the patient to be outside of this FOV. When using CT, the height of the imaging couch should ideally be set to position the patient centrally in the CT FOV to minimize the potential for CT truncation. However, it is accepted that there can also be a tradeoff against setting the bed height to maintain good quality auto-contouring for the SPECT scan, particularly for systems with only radial detector-orbit adjustment.

The second possible source of truncation of a CTderived attenuation map is the zoom factor that is applied to the attenuation map, which is the same as that applied to SPECT image. This can occur even if the original CT does not contain any truncation and is dependent on the choice of zoom factor.

Truncation is worst when it occurs predominantly on the left-hand side of the patient. This is because the iterative reconstruction process produces estimated projections to match those acquired by the SPECT camera, which will normally be RAO to LPO. For example, if the patient has their right arm by their side, and it is either partially or fully truncated in the attenuation map, this will have no impact on the attenuation calculation, as no rays would pass through the right arm if acquiring a standard $180^{\circ}$ orbit from RAO to LPO. Hence, if there is a risk of truncation, it is better to offset the patient slightly to their right on the imaging couch. This will bring the left side of the patient towards the

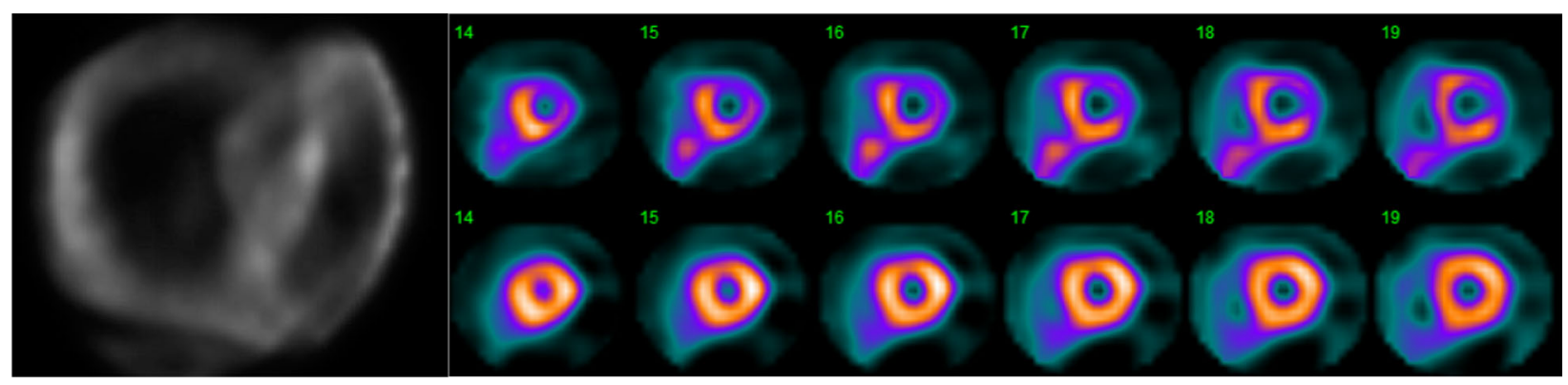

Figure 16. Example of truncation of a Gd-153 transmission image to the patient's left-hand side and resultant artifact on the attenuation-corrected images (top). 
center of the CT FOV and reduce the possibility of truncation to the left-hand side of the patient.

In patients with very large body habitus, it may be inevitable that truncation of the transmission data will occur. In these cases, care must be taken to ensure consistent patient positioning and bed height to maintain the amount of truncation between stress and rest images. If the degree of truncation differs at stress and rest, the attenuation correction will not be consistent, and this may lead to false interpretation of the images.

Misregistration. For sequential SPECT and CT scans acquired on hybrid SPECT/CT equipment, patient motion either during the SPECT scan itself or the transition between the SPECT and the CT or during the CT scan can cause a spatial misregistration between the SPECT image and the attenuation map. The attenuation coefficient of lung tissue for $140-\mathrm{keV}$ gamma rays is approximately one quarter that of soft tissue. The proximity of the myocardium to lung tissue means that even small misregistration where the myocardial SPECT image overlies the lung tissue will result in substantial artifacts in attenuation-corrected images due to the incorrect calculation of attenuation. Figure 17 demonstrates the effect of misregistration. It is essential that the registration between the SPECT and CT is assessed and corrected, if necessary. All vendors offer software tools to interactively adjust alignment in all three directions $(x, y$, and $z)$. It should also be noted that any adjustments to the SPECT and CT registration must be performed after any movement correction has been performed on the SPECT projection data for Anger camera. This is typically performed on the SPECT scanner acquisition console. If images are degraded due to physical movement during the CT scan, software corrections may not be helpful to salvage the images.

\section{Solid-State Cameras}

Reconstruction. Reconstruction algorithms on the CZT systems are tightly coupled with the hardware design of the detector. The new solid-system scanners offer much higher system sensitivity, due to the optimized detector geometry for cardiac acquisition and high sensitivity of the collimation. The high-collimator sensitivity would usually compromise image resolution but resolution recovery (as described in section "Resolution recovery") by accurate detector/collimator modelling, as well as selectively focusing the detectors on the heart, using patient-specific rotation angles in case of fast-rotating parallel collimators, allow these new scanners to maintain or improve overall spatial resolution, at the same time as the collimator sensitivity is increased. ${ }^{6,84}$ Additionally, to reduce image noise, the iterative reconstruction includes the regularization process between the iterations, which is optimized for MPI, usually without need for any adjustments by the user. Typically, mild 3D post-filtering is additionally applied after the reconstruction process. Reconstruction methods may also utilize "heart-prior" information during the iterative process, assuming expected distribution of the photon activity in myocardial perfusion region. Care must be exercised with this option in cases when myocardial distribution is unusual. Overall, these reconstruction improvements for the new dedicated cardiac scanners allow much shorter acquisition times or very low-dose imaging protocols. Shorter imaging times often lead to the additional benefit of reducing patient motion during scans and increasing patient comfort.

List mode and motion correction. By default, CZT systems obtain the raw acquisition data in list-mode format. The conventional projection data are not available. Simulated projection views can be reconstructed and used for the assessment of the heart position in the FOV (but not for the assessment of motion). Additionally, data from individual detectors could be displayed for QC. The list-mode data allow for retrospective gating and limiting the time of reconstructed data to specific portion of the scan. This may be helpful, for example, in eliminating portions of the scan with motion. Patient motion could be visually determined by cine replay of the 3D short-time dynamic frames - rather than by reviewing cine display of projection views, as is done with conventional cameras. Some vendors provide automated tools for such 3Dmotion detection and correction. Studies have shown that patient motion on this new camera will produce clinically significant image artifacts, and 3D correction from the list-mode data can ameliorate these artifacts. ${ }^{85}$ Because list-mode data can be used retrospectively to analyze errors and possibly correct reconstruction errors, these files should be stored with other patient images for possible future reprocessing.

Artifact mitigation with new scanners. The new dedicated cardiac cameras are not usually equipped with the hardware for the $\mathrm{AC}$, and most of the users of these new systems do not use AC. Furthermore, due to limited FOV of the new systems, it is possible to truncate heart data on some views, leading to truncation artifacts, especially in larger patients. ${ }^{86}$ In addition, a unique collimator/detector geometry can lead to $3 \mathrm{D}$ sensitivity variations. ${ }^{85}$ Therefore, attenuation of photons and other sources of imaging artifacts need to be recognized correctly on these systems by the users. To address this issue, new protocols and new analysis schemes have been developed in which two sequential scans in two patient positions (supine/upright or supine/ prone-depending on the scanner) are performed, 

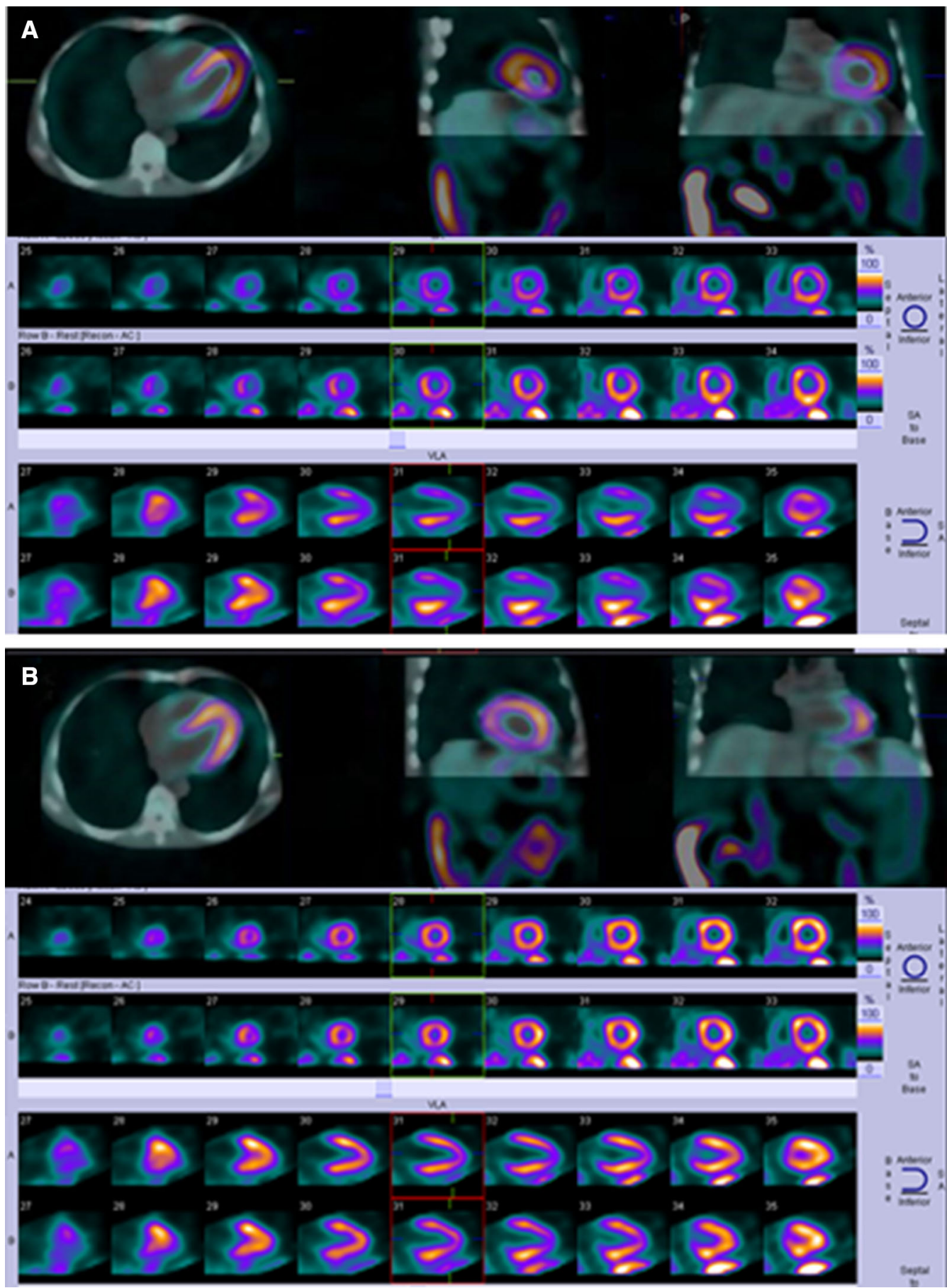

Figure 17. Example of misregistration on (A) SPECT perfusion, and (B) the repeat SPECT image after correction of misregistration. CT image shows misregistration between perfusion and CT transmission image, mainly along with anterolateral wall and apex. The resultant SPECT images show a reversible perfusion defect from the anterolateral wall extending to apex. Following correction of misregistration, the defect is no longer present.

allowing differentiation of true perfusion defects from artifacts, for the systems without AC hardware..$^{20,21,87}$ This 2-position imaging is facilitated by fast imaging times allowed by these systems. Two sequential scans can also aid in the recognition of truncation, motion or other types of artifacts related to the acquisition on the new systems, because, typically, patient position will change the appearance of these artifacts. 


\section{Reorientation}

A critical phase of myocardial processing is reorientation of tomographic data into the natural approximate symmetry axes of an individual patient's heart. This is performed either manually or automatically and results in sectioning the data into vertical longaxis, horizontal long-axis, and short-axis planes (see Figure 18, Standard tomographic slices orientation, A). Long-axis orientation lines should be parallel to longaxis walls of the myocardium and should be consistent between rest and stress studies. Inappropriate plane selections can result in misaligned myocardial walls between rest and stress data sets, potentially resulting in incorrect interpretation (see Figure 18, graphic of incorrect axis selection error, B). It is crucial that all axis choices be available as QC screens, and that these are reviewed by both the technologist and the physician who read each study prior to image interpretation to verify the axes were selected properly.

\section{Cine Review}

The most important post-acquisition QC procedure is to view the raw tomographic data in endless-loop cine mode. This presentation offers a sensitive method for detecting patient and/or heart motion, the presence and location of overlying breast tissue that may result in attenuation artifact, diaphragmatic elevation that may also result in attenuation artifact, and adjacent abdominal visceral activity, which may scatter into the inferior wall of the heart. All these findings may result in artifacts in the reconstructed images. Review of the raw tomograms in cine mode is performed twice: once by the technologist immediately after the acquisition before the patient leaves the imaging suite, and again by the

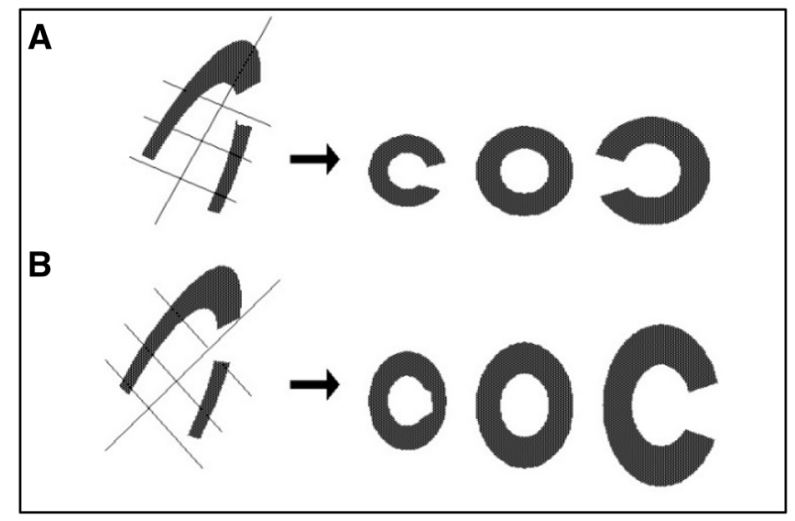

Figure 18. Standard tomographic slices orientation. (A) Correct. (B) Incorrect. physician during image interpretation. For gated studies, usually it is only the sum of all gated tomograms that is reviewed in this manner; but cine review of all tomographic data (i.e., projections through all phases of the cardiac cycle) may alert the observer to gating errors due to arrhythmias manifested by intermittent data drop-out, i.e., "flashing" of the images. A full display of all-count versus projection-curve data may be helpful (Figure 19). Display and review of the beat histogram (relative number of beats collected at specific R-R intervals) may also assist in determining if observed abnormal cardiac wall motion is real or artefactual. This display may also be helpful to detect gating errors that may confound the accuracy of calculated functional parameters. In addition, cine reviews occasionally show abnormalities in the abdomen or thorax, such as renal cysts or abnormal focally increased uptake that may be suspicious for neoplasm or other abnormalities, such as hepatosplenomegaly, hiatal hernias, etc.

\section{Recommendations for SPECT MPI Processing}

- ASNC recommends use of iterative reconstruction, advanced reconstruction methods, and attenuation correction when available or 2-position imaging to optimize image quality and improve diagnostic utility of SPECT MPI.

\section{INTERPRETATION}

\section{General Comments}

Prior to image interpretation, the technologist, basic scientist, and/or physician should ascertain that all camera QA parameters, including flood-field uniformity, linearity, and COR are acceptable. The interpretation of SPECT myocardial perfusion images should be performed in a systematic fashion to include: (1) evaluation of the raw images or the reconstructed maximum intensity projection image (MPI, for CZT scanners) in cine mode, and a review of the sinogram and linogram images, to determine the presence of potential sources of image artifact and the distribution of extracardiac tracer activity (as discussed in the prior paragraph); (2) interpretation of images with respect to the location, size, severity, and reversibility of perfusion defects, as well as cardiac chamber sizes, and the presence or absence of increased pulmonary uptake (especially ${ }^{201} \mathrm{Tl}$ ); (3) evaluation of the results of quantitative perfusion analysis; (4) evaluation of functional data obtained from the gated images; and (5) consideration of clinical data, stress ECG and hemodynamic data that may influence the final interpretation of the study. All 


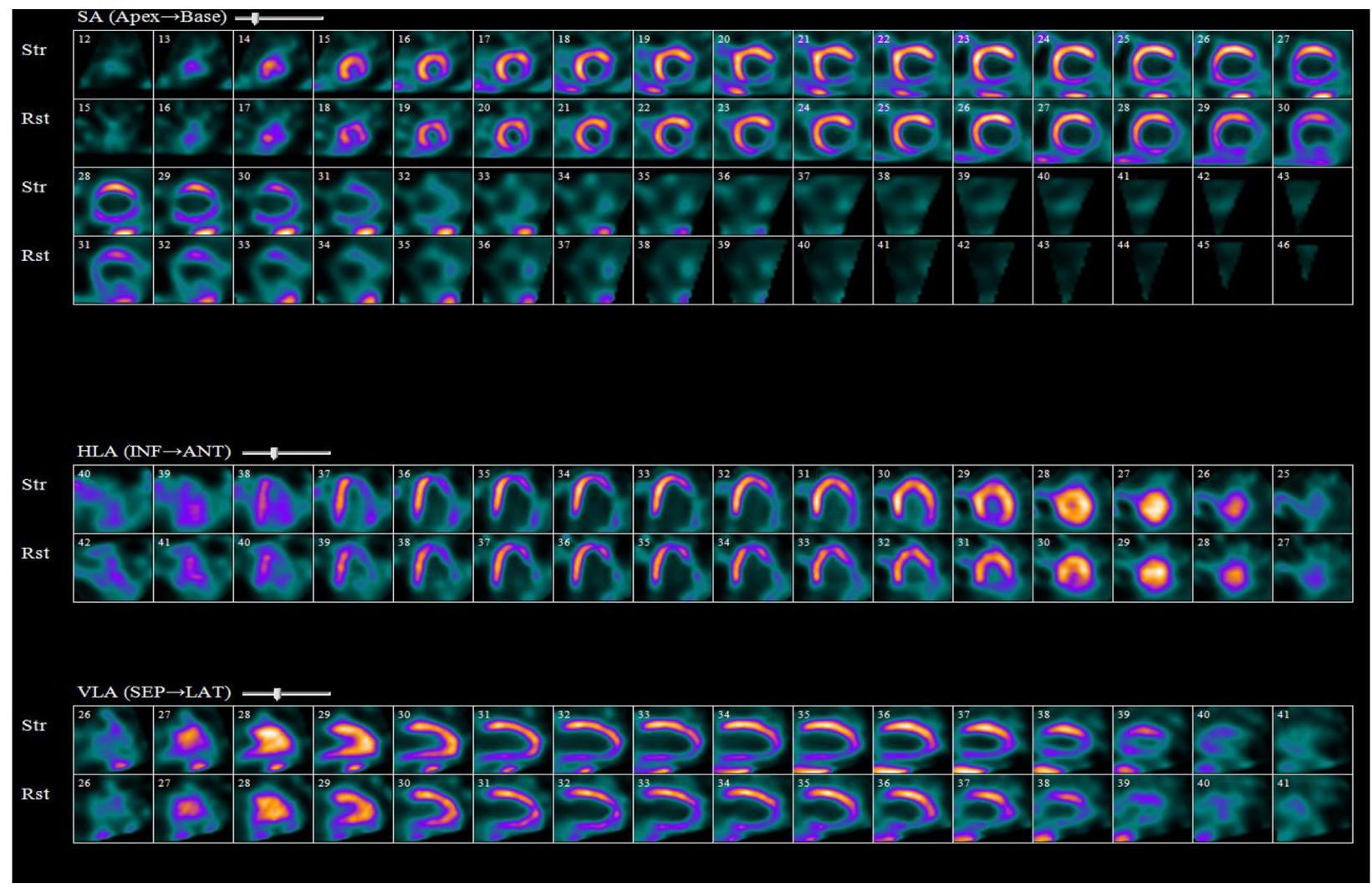

Figure 19. Standard display of stress and rest myocardial perfusion images. Stress and rest images are displayed in alternate rows. Short axis images (top 4 rows) are displayed from apex to base (left to right, row 1 continued on row 3 , and row 2 continued on row 4). Horizontal long axis images are displayed from the inferior wall to the anterior wall (left to right). Vertical long axis (VLA) images are displayed from septum to the lateral wall (left to right). These images demonstrate a large and severe perfusion defect in the entire inferior wall, the mid and basal inferolateral walls and the basal anterolateral walls that was fixed.

these factors should be systematically evaluated prior to generation of a final clinical report.

\section{Display}

Recommended medium for display. A computer display with commercially approved cardiac MPI software is recommended for image review and interpretation. Review of MPI images from film and paper hard copies is not recommended. A gray-scale translation table is generally preferred to color tables because the gray scale is linear and provides more consistent grades of uptake whereas most color scales may have artificially set transitions at various thresholds, but the choice of gray- vs color-scale is also dependent on the familiarity of the individual reader with a given translation table. ${ }^{88}$ A logarithmic scale may be used for evaluating regions of lower count density, such as softtissue uptake and the right ventricle (RV) but should never be used for primary interpretation of regional LV uptake. $^{88}$

Conventional slice display of SPECT images. Three sets of tomographic images should be displayed: (1) short-axis; (2) vertical long-axis; and (3) horizontal long-axis (Figure 19). The short-axis images, slices perpendicular to the long axis of the LV, should be displayed with the apical slices to the left and the base at the right. The vertical long-axis images, slices parallel to the septum, should be displayed with septal slices on the left and the lateral slices on the right. Similarly, the horizontal long-axis images, slices parallel to the inferior wall, should be displayed with inferior slices on the left and anterior slices on the right. It is also recommended, for purposes of comparison of sequential images (e.g., stress and rest, rest and redistribution), that the sequential images should be displayed aligned and adjacent to each other, with stress followed by rest perfusion, either in rows or columns. If three sets of 
images are available (for example, rest supine, stress supine and stress upright) then the most commonly used MPI order for display is stress supine, stress upright, and rest supine.

There are two widely used approaches to image normalization. The first approach is "series normalization', where each series (vertical, horizontal, short-axis) may be normalized to the brightest pixel in the entire image set. This is the recommended method to evaluate the extent and severity of perfusion defects. The drawbacks of this approach are its sensitivity to focal hot spots, the frequently poor visualization of normal structures at the base and apex of the LV, and the lack of an ideal display of each individual slice. The other approach called frame normalization is a reasonable option where the brightness of each slice is unrelated to the peak myocardial activity in each individual slice. This approach can help avoid attenuation artifacts particularly in the mid and basal segments of the myocardium and has been shown to result in both high sensitivity and specificity for CAD detection. ${ }^{89}$

Three-dimensional display. Commercial software programs allow creation of 3D displays of regional myocardial perfusion. These displays may help less experienced readers identify coronary distributions associated with perfusion defects, but 3D displays should be used only as an adjunct to, not a replacement for, the conventional image interpretation previously described.

\section{Evaluation of the Images for Technical Sources of Error}

Patient motion. The raw planar images should be reviewed in a rotating format (grey or color scale) for possible sources of attenuation artifact and for the presence of patient motion. For conventional cameras, a cine display of the planar projection data is highly recommended because motion in both the vertical (craniocaudal) and horizontal (side-to-side) axes are readily detectable. Additionally, a static sonogram or linogram may be used to detect patient motion. Vertical ( $y$-plane) motion is more readily detected on the linogram, whereas horizontal motion, often overlooked on the rotating cine images, is easily observed on the sinogram (Figure 20). The reader should be familiar with the normal appearance of raw planar images and be able to identify motion artifact. For novel camera designs, such as multi-detector high-sensitivity CZT systems, planar projections may not be available, and the reader must inspect the sinograms or 3D motion from short-duration SPECT frames. Although software programs are available for the quantitation and correction of patient motion, in patients who have had an MPI

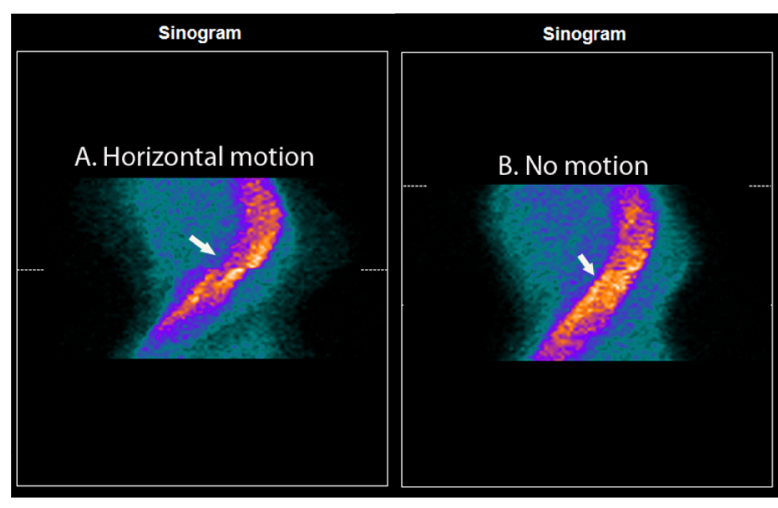

Figure 20. Sinogram showing (A) horizontal motion and (B) no motion.

with ${ }^{99 m}$ Tc-based perfusion agent with negligible myocardial redistribution (e.g., ${ }^{99 \mathrm{~m}} \mathrm{Tc}$ sestamibi or ${ }^{99} \mathrm{~m}$ Tc tetrofosmin), image acquisition should be repeated when significant motion is detected. Repeat imaging in prone position may be considered to minimize motion. The effect of patient motion on the final reconstructions is complex. ${ }^{90,91}$ Generally, vertical (i.e., craniocaudal) motion has less of an effect on the accuracy of the study than horizontal (side-to-side) motion, especially when the heart returns to the same baseline. Vertical motion is also much easier to correct manually or with semi-automated software. Rotation currently cannot be corrected either manually or with available motion-correction software. Because motion correction software may sometimes introduce motion artifact, corrected raw planar images should be evaluated for adequacy of the correction.

Attenuation artifacts and attenuation correction. The cine display of the planar projection images is also recommended for the identification of sources of attenuation, the most common being the diaphragm in men and the breast in women. ${ }^{92}$ Breast attenuation artifact is most problematic when the left breast position varies between the rest-and-stress images (i.e., 'shifting breast attenuation artifact'). When the apparent perfusion defect caused by breast attenuation artifact is more severe on the stress images than on the resting images, ischemia cannot be excluded. Breast attenuation artifact can be confirmed by repeating the acquisition with the left breast repositioned. Diaphragmatic attenuation and breast attenuation may also be addressed by imaging the patient prone. Hardware and software for attenuation and scatter correction are commercially available and may obviate or at least mitigate these common attenuation artifacts. Breast and diaphragmatic attenuation artifacts are less apparent with upright imaging. 
Evaluation of myocardial perfusion images corrected using sealed radionuclide source or CT based AC is described in section "Final Interpretation of MPI with Clinical and Stress-Test Data'. As with the interpretation of non-attenuation-corrected images, it is essential that the interpreting physician be familiar with the specific AC system that is being used. ${ }^{93-95}$ Because of the complexity of current correction methods in SPECT, it is recommended that the non-attenuation-corrected images be interpreted along with the attenuation-corrected-images.

Reconstruction artifacts. Intense extracardiac tracer activity in close proximity to the heart (e.g., superimposed bowel loops or liver activity) may create artifactually increased uptake in adjacent myocardium that could mask a perfusion defect or be misinterpreted as reduced uptake in remote myocardial segments due to image normalization to the artifactually "hot" area. Non-superimposed but adjacent extracardiac activity may also cause a negative reconstruction artifact with FBP image reconstruction (ramp filter artifact, section "Filtered backprojection", Figure 7), resulting in an apparent reduction in activity in the adjacent myocardial segment. There is currently no reliable correction for such artifacts, and attenuation algorithms can even exacerbate them if, for example, the abdominal wall has partially attenuated the extracadiac radiotracer activity on the non-attenuation-corrected images. These artifacts may be less problematic with iterative as opposed to FBP reconstruction techniques and with camera systems that have higher spatial resolution. These artifacts can often be eliminated by repeating the acquisition after the activity level in the adjacent extracardiac structure has decreased. Some methods to decrease extracardiac activity include delayed imaging, food, water, or milk intake, and prone imaging.

Myocardial count statistics. Many factors are involved in the final count density of perfusion images, including body habitus, exercise level achieved, administered radiotracer activity, acquisition time, energy window, and collimation. Apparent perfusion defects can be artifactually created simply because of lowimage count density, a particular problem with ultralow radiation dose imaging protocols. The interpreting physician should make note of the count density in the planar projection images because the quality of the reconstructed data is a direct reflection of the count-rates measured on the raw data. While the optimal counts for conventional $\mathrm{NaI}$ cameras are not well defined, greater than $1,000,000$ counts/LV are shown to be adequate for novel CZT detector cameras. ${ }^{21}$

\section{Initial Image Analysis and Interpretation}

Ventricular dilation. Before segmental analysis of myocardial perfusion, the reader should note whether there is LV enlargement at rest or post-stress. Dilation of the LV on both the stress and resting studies usually indicates LV systolic dysfunction, although it may be seen in volume overload states (e.g., severe mitral or aortic regurgitation) with normal ventricular systolic function.

An increased stress-to-rest LV cavity ratio, transient ischemic dilation (TID), also referred to as transient cavity dilatation (TCD), has been described as a marker for high-risk coronary disease. ${ }^{96-98}$ Transient ischemic dilatation on SPECT MPI, imaged about 30 minutes after completion of stress testing, is more likely to represent apparent dilatation of the ventricle from diffuse subendocardial ischemia ${ }^{99,100}$ or microvascular disease in the absence of epicardial coronary disease. Transient ischemic dilation is typically evaluated on static images; if TID is apparent on qualitative assessment, TID ratio may be quantified. ${ }^{101,102}$ Normal limits by quantitation will depend on perfusion imaging protocol, the image processing parameters, and the software algorithm used. To reliably determine LV volumes and the TID ratio, it is critical that the endocardial border is accurately tracked and that the valve plane is accurately determined and consistent in the stress and rest images. Imprecise valve plane determination is a frequent cause of erroneous TID values.

Lung uptake. The presence of increased lung uptake after ${ }^{201} \mathrm{Tl}$ perfusion imaging has been described as an indicator of poor prognosis and should therefore be evaluated in all patients when using this perfusion agent. ${ }^{99,100,103}$ No clear consensus has emerged as to the significance of lung uptake with ${ }^{99 \mathrm{~m}} \mathrm{Tc}$-based perfusion agents, although increased lung ${ }^{99 \mathrm{~m}} \mathrm{Tc}$ tracer uptake may provide a clue to the presence of resting LV systolic dysfunction in patients who are not candidates for gatedSPECT imaging due to severe arrhythmias.

Right ventricular uptake. Right ventricular uptake may be qualitatively assessed on the raw projection data and on the reconstructed data. There are no established quantitative criteria for RV uptake, but in general, the intensity of the RV is approximately $50 \%$ of peak LV intensity. RV uptake increases in the presence of RV hypertrophy, most typically because of pulmonary hypertension. ${ }^{104}$ The intensity of the RV may also appear relatively increased when LV uptake is globally reduced. ${ }^{105}$ Regional abnormalities of RV uptake may be a sign of ischemia or infarction in the 
distribution of the right coronary artery. The size of the RV should also be noted qualitatively, as RV dilation can provide a clue to the presence of right heart volume overload due to conditions, such as atrial septal defect or severe tricuspid regurgitation.

Noncardiac physiological and pathological findings. Compared to rest MPI, splanchnic tracer activity on stress MPI is generally increased following pharmacologic stress with dipyridamole, adenosine, regadenoson, or dobutamine (due to splanchnic hyperemia); it is, however, reduced following adequate exercise stress with greater than $85 \%$ maximum predicted heart rate (due to hyperemia to the exercising muscles). Careful review of the raw images and familiarity with normal isotope distribution (e.g., liver gall bladder, intestines) helps to recognize abnormal focal extra-cardiac uptake in breast, axilla, thyroid, mediastinum and abdomen. ${ }^{106}$ Both ${ }^{201} \mathrm{Tl}$ - and technetiumbased agents can be concentrated in tumors, and uptake outside the myocardium may also reflect other unexpected pathology. ${ }^{106}$ However, the sensitivity and specificity of MPI for diagnosing non-cardiac conditions have not been well established. Review of the raw image data can also identify the presence of pericardial effusions, and particularly when large, recognized as a circumferential radiolucent region around the heart.

Perfusion defect location. Myocardial perfusion defects are evaluated by visual analysis of the reconstructed slices. The location of the perfusion defects is characterized using the 17-segment heart model or as using specific myocardial walls (apical, anterior, inferior, and lateral). Standard segment nomenclature is recommended (see the segmentation models depicted in Figure 21 and Table 7). ${ }^{107}$ The myocardial segments may be roughly assigned to coronary arterial territories as indicated in Figure 21. Readers must be aware of the considerable variation in the coronary anatomy of the diagonal, circumflex, and right coronary arteries. For example, an inferior defect may represent disease in either the right coronary artery or the left circumflex coronary artery territory. However, an inferior defect extending into the basal inferoseptum more likely represents posterior descending CAD, while an inferior defect extending in to the inferolateral segments represents posterolateral CAD. Likewise, an anterolateral defect extending into the anterior wall may represent diagonal $\mathrm{CAD}$, while anterolateral defect extending into the inferolateral wall may represent obtuse marginal CAD. An inferolateral defect extending
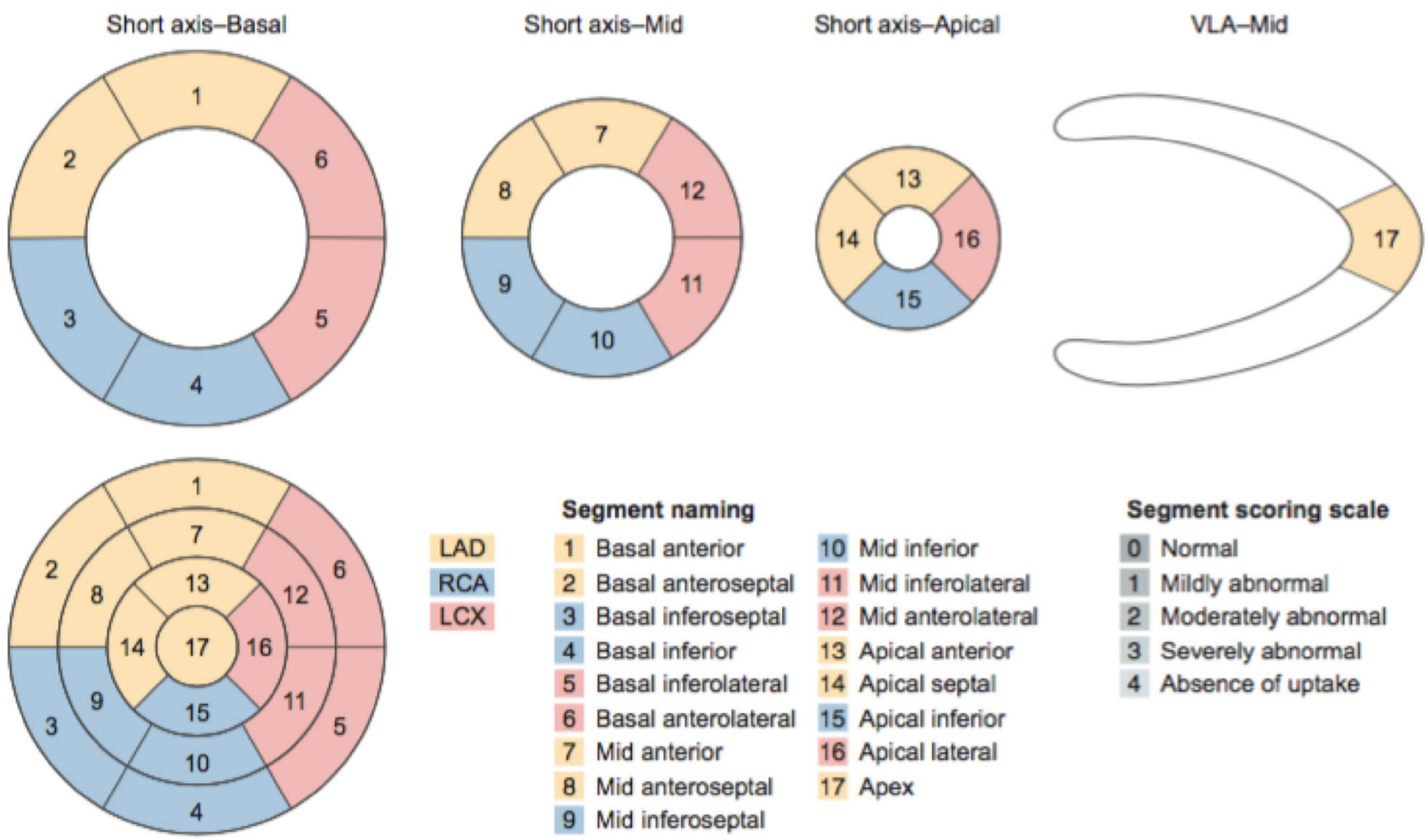

Figure 21. Left ventricular segmentation and scoring. Adapted and reprinted with permission from the American Society of Nuclear Cardiology; originally presented in Cerqueira MD, et al. $J$ Nucl Cardiol 2002;9:240-5. ${ }^{107}$ 
Table 7. The five-point scale for scoring

\begin{tabular}{lc}
\hline \multicolumn{1}{c}{ Category } & Score \\
\hline Normal perfusion & 0 \\
Mild reduction in counts $(10 \%-<25 \%)$ & 1 \\
Moderate reduction in counts $(25 \%-50 \%)$ & 2 \\
Severe reduction in counts $(\geq 50 \%)$ & 3 \\
Absent radiotracer uptake (background level of counts) & 4 \\
\hline
\end{tabular}

into the basal anterolateral wall may represent proximal left circumflex or first obtuse marginal CAD. To a lesser degree, the coronary arterial perfusion to the apex may also be variable, and the entire apex is often supplied by the LAD alone, and the defect may extend into the midinferior wall (wraparound LAD).

Perfusion defect severity and extent. Qualitative. Defect severity is typically expressed qualitatively as mild, moderate, or severe. Defect extent may be qualitatively described as small, medium, or large, but, defect extent is better estimated semiquantitatively (see below). Defects whose severity and extent do not change between stress-and-rest images are categorized as "fixed" or "'nonreversible." When perfusion defects are more severe and/or extensive on stress compared to rest images, a qualitative description of the degree of reversibility is required.

Semiquantitative. In addition to the qualitative evaluation of perfusion defects, semiquantitative interpretation of MPI is recommended. ${ }^{107,108}$ This approach standardizes the visual interpretation of scans, reduces the likelihood of overlooking significant defects, and provides an important semiquantitative index that is applicable to diagnostic and prognostic assessments and to guide therapy.

Defect severity in each of the 17-segments on the stress, rest, or redistribution images (as applicable) is scored semiquantitatively using a 0 to 4 score as shown in Table 7. Defect severity can also be expressed as mild ( $10 \%$ to $<25 \%$ reduction in counts), moderate ( $25 \%$ to $<50 \%$ reduction in counts), severe ( $\geq 50 \%$ reduction in counts), or absent tracer uptake (background counts).

Defect extent may be described semiquantitatively as small (involving 1 to 2 segments), medium (involving 3 to 4 segments), or large (involving $\geq 5$ segments); Also, small, medium, and large defects involve $<10 \%$, $10 \%$ to $20 \%$, and $\geq$ to $20 \%$ of the $\mathrm{LV}$, respectively.

The use of a scoring system provides a reproducible semiquantitative assessment of defect severity and extent. ${ }^{109}$ A consistent approach to defect severity and extent are clinically important because both variables contain independent prognostic information. ${ }^{110}$ Points are assigned to each segment according to the count density of the segment.

In addition to individual segmental scores, it is recommended that summed scores and percent myocardium metrics be calculated. The summed stress scores equal the sum of the stress scores of all the segments, and the summed rest score equals the sum of the resting scores (or redistribution scores) of all the segments. ${ }^{110,111}$ The summed difference score equals the difference between the summed stress and the summed resting or redistribution scores and is a measure of perfusion defect reversibility, reflecting inducible ischemia, or hibernating myocardium, respectively. In particular, the summed stress and rest scores have been shown to have significant prognostic power. ${ }^{111-114}$ Before scoring, it is necessary for the interpreting physician to be familiar with the normal regional variation in count distribution of myocardial perfusion SPECT in men and women. The visual scores can be used to generate percent of involved myocardium. For a 17 -segment model with a 0 to 4 scoring scheme, the maximal possible score is 68 , and percent myocardium abnormal, ischemic or scarred is calculated as the following: (summed score $\times 100 / 68) .{ }^{115}$ The current generation software also provides for percent myocardium abnormal, ischemic or scarred values for SPECT MPI but must be confirmed by interpreting physicians due to possible errors in the identification of landmarks and interference from extracardiac activity that may affect these values. The semiquantitative metrics are recommended to guide appropriate use of coronary revascularization. ${ }^{2,116}$

Quantitative. Quantitative analysis of static perfusion images is useful to supplement visual interpretation. ${ }^{117,118}$ Recent studies have shown to have similar diagnostic accuracy to semiquantitative scoring. ${ }^{65}$ Most techniques of quantitative analysis are based on radial plots of short-axis slices and analyze the apex separately. These plots are then normalized to allow comparison to a normal gender-specific and cameraspecific database. Defect severity can be defined based on the patient's regional myocardial tracer activity 
compared to the mean regional activity of a normal database. Quantitation of the stress perfusion is compared to the resting perfusion to assess the extent and severity of ischemia. It is customary to use separate, normal databases specific to the imaging camera, the reconstruction method, the patient's gender, as well as the perfusion agent used. ${ }^{119}$

This quantitative analysis is typically displayed as a "bullseye" or polar plot. The quantitative programs are effective in providing an objective interpretation that is inherently more reproducible than visual analysis, eliminates the variability of the appearance of a defect when viewed in different media (with different radiotracers) and different translation tables, and is particularly helpful identifying subtle changes between two studies in the same patient. ${ }^{109,120-122}$ Quantitative analysis also serves as a guide for the less-experienced observer who may be uncertain about normal variations in uptake. Defect extent may be quantitatively expressed as a percentage of the entire $\mathrm{LV}$, or as a percentage of individual vascular territories, the latter being less reliable because of the normal variations in coronary anatomy. Defect severity may be quantitatively expressed as the number of standard deviations by which the segment varies from the normal range for that particular segment. Segmental scores also can be computed automatically mimicking semiquantitative visual scoring. Defect reversibility may also be expressed as a percentage of the entire LV or of a vascular territory. An integrated measure of defect extent and severity (total perfusion deficit) may provide valuable diagnostic ${ }^{65}$ and prognostic information. ${ }^{123}$

Despite these advantages, quantitative programs have certain limitations. They rely on adequate myocardial contours, which can sometimes be challenging in scans with very severe perfusion defects or with excess subdiaphragmatic activity. These programs are not currently sophisticated enough to differentiate perfusion defects from artifact in all cases. Because of imaging artifacts and the underestimation of ischemia with current SPECT perfusion tracers, there will always be difficulty in distinguishing normal subjects and patients with mild perfusion defects. Quantitative analysis should only be used as an adjunct to, and not a substitute for, visual analysis.

Reversibility. Reversibility of perfusion defects may be categorized qualitatively as partial or complete, the latter being present when the activity in the defect returns to a level comparable to surrounding normal myocardium. The semiquantitative scoring system may be used to define reversibility as a greater than or equal to 2-grade improvement or improvement to a score of 1 . Reversibility on a quantitative polar plot will depend on the specific software routine in use and the normal reference databases used in the program. Reversibility is typically described as improvement to a pixel density of less than 2.5 standard deviations from the normal reference redistribution or resting database. Quantification of reversibility without normal databases is also possible. $^{124}$ The number of pixels in the images that need to improve perfusion to determine defect reversibility is arbitrary.

So-called "reverse redistribution" may be seen in stress-delayed ${ }^{201}$ TI imaging and in rest-delayed technetium-sestamibi imaging. ${ }^{125}$ Reverse redistribution refers to segments with decreased or normal intensity on the initial set of images that show even less relative intensity on the delayed images. The interpretation of the finding remains controversial, and in most cases, this image pattern is the result of a slightly more severe attenuation artifact on low-dose resting images compared to high-dose stress images. Reverse redistribution pattern has also been reported to occur in myocardial segments with a mixture of viable and nonviable myocardium that are supplied by patent infarct-related arteries. $^{126}$

\section{Gated Myocardial Perfusion SPECT}

Gated-perfusion SPECT to assess ventricular function is integral to myocardial perfusion SPECT. ${ }^{56,57} \mathrm{~A}$ systematic approach to the display and interpretation of the ventricular function derived from gated SPECT is important.

Gated SPECT display. Multiple ventricular slices should be displayed for visual assessment of regional wall motion and systolic wall thickening. At a minimum, a display of apical, mid, and basal short-axis; a mid-ventricular horizontal long-axis; and a midventricular vertical long-axis slice(s) should be viewed. Ideally, the software should allow the user to scroll through any of the slices in any axis in cine mode. Each view should be normalized to the series of end-diastolic to end-systolic slices to maintain the apparent count density changes during the cardiac cycle that reflect myocardial wall thickening. Software algorithms that automatically define epicardial and endocardial borders, and calculate ventricular volumes and EF, should be applied. Regional wall motion should be interpreted preferably with a gray-scale display. The physician should review the assigned endocardial and epicardial contours to ensure accuracy of the volumes and LVEF, and may use these contours to analyze wall motion. But wall motion without computer-derived edges should be reviewed. Regional wall thickening may be analyzed in gray scale or in a suitable color scheme, although color displays may make it easier to appreciate changes in count intensity. 
Gated SPECT QC. All the QA procedures for routine SPECT are applicable to gated SPECT. Attention should be paid to the definition of the base of the $\mathrm{LV}$. Incorrect selection of the valve plane may result in erroneous determination of LV volumes and LVEF. For gated SPECT it is also essential to evaluate the adequacy of the ECG gating. ${ }^{58}$ The most common manifestation of poor gating is the appearance of a flashing pattern on the rotating planar projection images that results from count loss in the later frames. Ideally, a heart-rate histogram should also be viewed to verify beat length uniformity. Inspecting the time-volume curve is particularly useful because gating errors may distort the curve. Another important aspect of QC is a visual or quantitative determination that the number of counts acquired in each frame of the gated study was adequate for assessment of function. Software that collects all counts into a separate bin for the summed image can minimize the effect that gating errors have on the summed image.

\section{Gated SPECT: regional wall motion} and thickening. Regional wall motion should be analyzed through standard nomenclature: normal, hypokinesis, akinesis, and dyskinesis. Hypokinesis may be further qualified as mild, moderate, or severe. A semiquantitative scoring system is recommended where 0 is normal, 1 is mild hypokinesis, 2 is moderate hypokinesis, 3 is severe hypokinesis, 4 is akinesis, and 5 is dyskinesis. Software algorithms can also aid physicians in semiquantitative scoring of wall motion and thickening. ${ }^{127}$

This scoring system is comparable to the 5-point scoring system used in both X-ray contrast and radionuclide ventriculography. As in any assessment of regional ventricular function, one must be cognizant of expected normal and abnormal variations, such as the reduced wall excursion at the base compared with the apex, the greater excursion of the basal lateral wall compared with the basal septum.

Normal myocardial wall thickness is below the spatial resolution of currently available SPECT systems. Because of the "partial volume effect," regional wall thickening can be estimated by the count increase from end diastole to end systole. It is more difficult to visually assess the severity of abnormality of myocardial wall thickening than it is to visually estimate abnormalities of wall motion. However, the evaluation of thickening with gated perfusion SPECT lends itself to quantitation because it is characterized by count changes.

Wall motion and wall thickening are generally concordant. The principal exception to this occurs in patients with left bundle branch block, post pericardiotomy, RV pacing, or after cardiac surgery where septal wall motion is frequently abnormal (paradoxical), but there is normal wall thickening. Rather than separately scoring wall motion and wall thickening, it is commonly accepted to incorporate the two findings into a single score, while noting the presence of discordance in wall motion and wall thickening when it occurs. Paradoxical septal motion can be identified by normal septal perfusion with abnormal wall motion on the quantitative wall motion polar plots. In addition to noting LV wall motion, wall thickening, and $\mathrm{EF}$, the function of the RV should also be noted. Quantitative normal databases are available for assessment of regional wall thickening.

Left ventricular ejection fraction and volumes. Left ventricular ejection fraction and LV chamber sizes should routinely be evaluated both qualitatively and quantitatively. ${ }^{128}$ Ejection fraction may be categorized as normal $(>55 \%$ to $<70 \%$ ), low normal $(50 \%$ to $55 \%)$, mildly $(45 \%$ to $<50 \%)$, moderately $(35 \%$ to $<45 \%)$, severely reduced $(<$ $35 \%$ ), or hyperdynamic $(>70 \%)$ but may also vary with software. Normal values for LVEF are significantly influenced by gender, and to a lesser extent by age and body weight. In women, LVEF calculation by gated SPECT by most software programs is significantly higher than in men, partly because women generally have smaller hearts than models used and there are greater errors, but perhaps this is due to physiological differences.

Volume may be categorized as normal, mildly, moderately, or severely increased. Left ventricular volumes and ejection fraction on gated-SPECT MPI are determined by tracing the contours of the myocardium on the tomographic slices and are highly reproducible and quantitative. ${ }^{129}$ Although both rest and stress MPI are gated with ${ }^{99 \mathrm{~m}} \mathrm{Tc}$ perfusion tracers, LVEF and volumes from the higher dose study are usually reported due to better quality of the images.

Integration of perfusion and function results. The results of the perfusion and gated-SPECT data sets should be integrated into the final interpretation. The wall motion is particularly helpful in distinguishing nonreversible (fixed) perfusion defects due to prior myocardial infarction from fixed defects due to attenuation artifacts. Fixed perfusion defects that do not show a corresponding abnormality of wall motion or myocardial systolic thickening are more likely to be due to artifacts, especially if the clinical data do not support prior infarction. ${ }^{92}$ The finding of normal regional wall motion and myocardial systolic thickening cannot be used to exclude ischemia in patients with reversible perfusion defects. Because the post-stress gated images are acquired more than 30 minutes after exercise or pharmacologic stress, stress-induced regional dysfunction will have resolved by the time the SPECT images are acquired, except in some cases of severe ischemia/ 
LV stunning as discussed below. Therefore, unless poststress stunning is present, ${ }^{130}$ in the great majority of cases post-stress regional wall motion is normal in patients with stress-induced ischemia.

In individuals with significant stress-induced ischemia, post-stress LVEF may not accurately represent resting LV systolic function. Post-ischemic regional ${ }^{130}$ or global stunning is a marker for high-risk CAD and increases the specificity of the MPI findings for CAD. ${ }^{131}$ Caution must be exercised, however, when interpreting changes in LVEF in several clinical scenarios. First, comparison of rest and stress LVEF with different radiotracer doses can be challenging. When there is a marked discrepancy in rest and stress LVEF in the absence of other high-risk markers, technical accuracy needs to be confirmed.

LV systolic dyssynchrony. Innovative software has been developed to measure LV systolic dyssynchrony using phase analysis of gated myocardial perfusion SPECT. ${ }^{132}$ Increasing myocardial counts during ventricular systole correlates with regional LV wall thickening and may be used to assess the pattern of systolic contraction. Data from the entire myocardium are used to generate a phase distribution map that may be displayed as a histogram or polar map. By means of a sampling theorem, temporal changes in LV myocardial contraction can be assessed using only 8-frames per cardiac cycle. This method provides temporal resolution equivalent to only $1 / 64$ th of the cardiac cycle. ${ }^{133}$ It has been reported that LV dyssynchrony measured by this method is useful to predict which patients will respond to cardiac resynchronization therapy (CRT). ${ }^{134}$ Although this radionuclide method is quite promising, there have been parallel advancements in echocardiography, magnetic resonance imaging, and cardiac $\mathrm{CT}$ in assessing LV dyssynchrony and patient response to CRT. ${ }^{135}$ Current literature, however, does not conclusively support a role for imaging-based evaluation of dyssynchrony to predict response to cardiac resynchronization therapy, and QRS duration remains a consideration for cardiac resynchronization therapy decisions. ${ }^{136}$

\section{Clinical Interpretation of AC SPECT Studies}

Interpretation of AC SPECT myocardial perfusion images follows a similar approach to that used for nonattenuation-corrected myocardial perfusion images with specific differences in normal radiotracer distribution, quality assurance methods, and image interpretation.

The normal distribution of perfusion tracers is significantly different in the attenuation corrected compared to non-attenuation-corrected images. Although generally relatively similar from system to system, the geometry of the imaging system, acquisition protocol, and processing algorithms can alter normal myocardial radiotracer distribution ${ }^{94}$ There can also be differences in normal distribution(s) related to patient gender and ventricular volume. The interpreting physician must be aware and account for these differences on a patient-bypatient basis when interpreting attenuation-corrected studies. Attenuation-corrected SPECT MPI studies generally have uniform regional activity in the anterior, septal, inferior, and lateral walls; but, mild reductions in apical and distal anterior activity are common (as with PET MPI). This finding is often more prominent in patients with larger hearts, in men, and when corrections are applied for resolution recovery and scatter correction.

Quality assurance of the attenuation-corrected images is required for each study. The quality of the transmission images from the current SPECT/CT systems consistently exceeds the quality of sealed source transmission images (radionuclide-TCT). The higher resolution the $\mathrm{CT}$ transmission images compared to the emission images increases the potential for misregistration of emission and transmission images and related artifacts. Co-registration of the transmission images (or mu maps) with the emission images must be ensured. Artifacts can result from movement (respiration or patient movement), misregistration of the transmission and emission images (see Figure 17), or intense extracardiac radiotracer uptake (amplified by the iterative reconstruction algorithms that are employed in AC reconstructions). While artifacts related to misregistration of transmission and emission images from respiratory changes can be corrected using software methods (see section "Misregistration"), those due to misregistration from patient motion (during transmission or emission imaging) may be more challenging to correct and may require repeat imaging.

After quality check, including review of rotating projection images and for extra-cardiac activity, patient motion, misregistration, attenuation corrected and nonattenuation-corrected images are displayed side by side, with displays of the normal activity distribution(s) and their variance distribution available as required for comparison. If this is not possible, the non-AC images should be interpreted first, then the AC images. Figure 22 provides an example of fixed perfusion defect in the inferior wall on the non-attenuation corrected images that normalized on the attenuation corrected images, suggesting inferior wall attenuation artifact. As in this example, typically, the final interpretation of the myocardial perfusion images is based on the findings on attenuation corrected images. However, when perfusion defects are seen only on the attenuation corrected images (normal non-attenuation corrected images), AC 


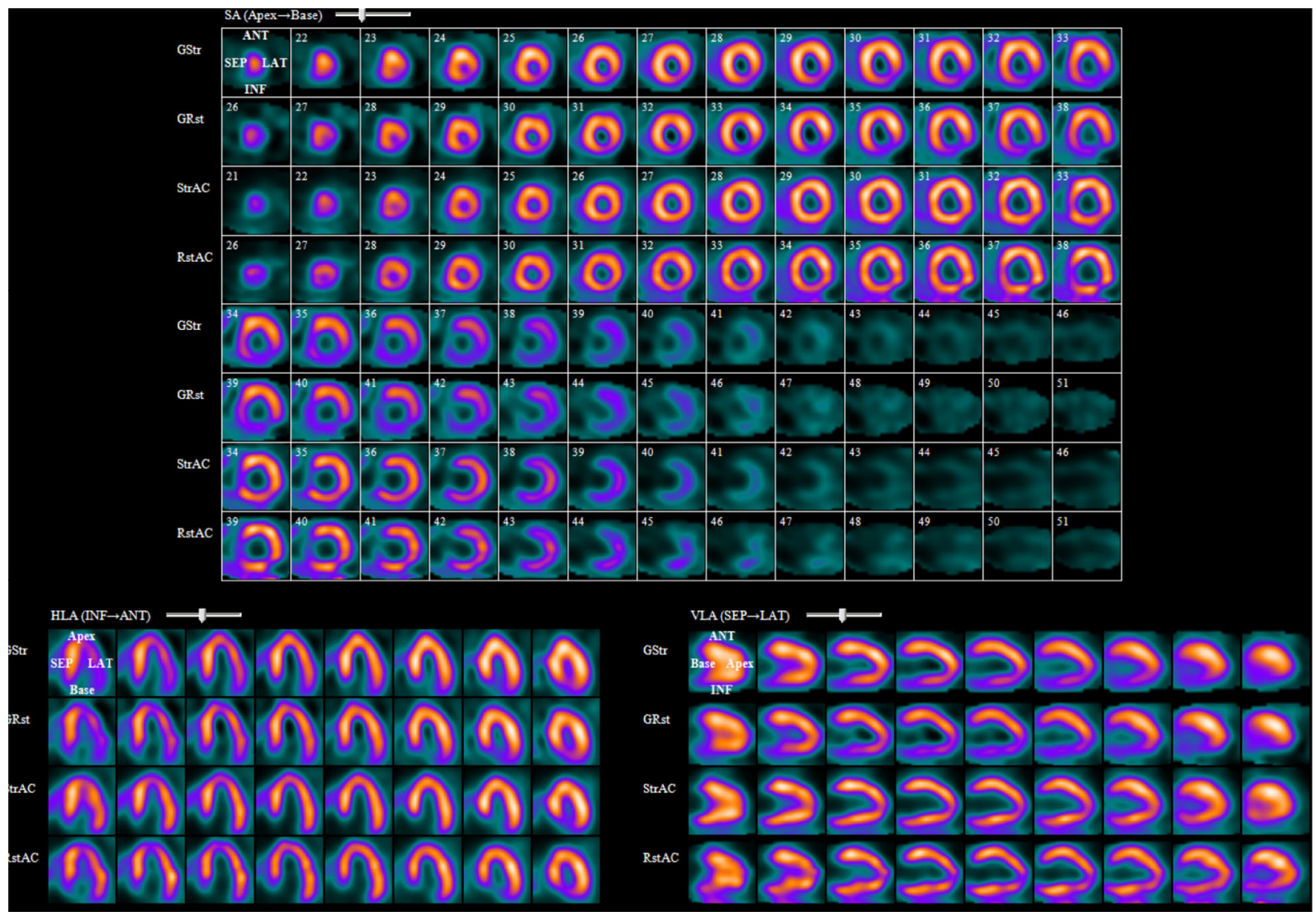

Figure 22. Value of attenuation correction of SPECT myocardial perfusion images. A 55-year old male with new onset atrial fibrillation and atypical chest pain underwent exercise same day reststress ${ }^{99 \mathrm{~m}}$ Tc-sestamibi SPECT MPI. Non-attenuation corrected images (first and second rows of the short axis, SA, horizontal long axis, HLA and vertical long axis, VLA images) demonstrate smallmoderate fixed defect in the inferior wall which normalized on the attenuation corrected images (third and fourth rows).

related artifacts need to be considered and excluded. Intense extra-cardiac activity can scatter counts into the inferior wall and mask/overcorrect inferior wall perfusion in attenuation corrected images and make the anterior wall apparently photopenic. Apical thinning, a normal variant, can be more pronounced with AC; fixed apical defects are considered abnormal when defects are severe perfusion, have corresponding wall motion abnormality, or corresponding perfusion abnormalities in left anterior descending territory. Misregistration in CT transmission/SPECT perfusion should also be evaluated as a cause of new AC defect particularly along anterior /anterolateral walls. After excluding artifacts, new AC perfusion defects can be considered abnormal if they conform to a coronary distribution. ${ }^{137}$

\section{Final Interpretation of MPI with Clinical and Stress-Test Data}

After a systematic image interpretation as discussed previously, perfusion images are reported in categories of normal, probably normal, equivocal, probably abnormal or abnormal. ASNC recommends a definitive reporting of the scan as normal or abnormal and minimizing use of probably normal or probably abnormal. $^{2}$ The category equivocal should be reserved for challenging cases (e.g., reversible anterior wall defect where variable breast attenuation artifact cannot be excluded). To avoid reader bias, the initial interpretation of the perfusion study should ideally be performed without any clinical information other than the patient's gender, height, and weight. All relevant clinical data 
should be reviewed after a preliminary impression is formed. The images can be reviewed again and a final impression reported taking into consideration the clinical data and electrocardiogram but, typically, without changing the preliminary impression by no more than one grade (e.g., probably abnormal to abnormal). In select cases of discordance, with normal MPI and high risk stress test findings (exercise induced ST elevation $>$ $1 \mathrm{~mm}$ in leads without $\mathrm{Q}$ waves, exercise induced drop in systolic blood pressure of $>10 \mathrm{~mm} \mathrm{Hg}$ from baseline, horizontal or downsloping ST segment depression $\geq 3$ $\mathrm{mm}$, or sustained ventricular tachycardia), evaluation of coronary anatomy may be considered. ${ }^{138}$ Guidelines for interpretation and reporting of myocardial perfusion SPECT are listed in Table 8.

\section{Myocardial Viability}

Detection of viable myocardium is important to identify patients with severe CAD and LV dysfunction who may benefit from coronary artery revascularization. Myocardial perfusion in dysfunctional myocardium can be normal (nonischemic cardiomyopathy or stunning) or abnormal (hibernating myocardium or scar). Myocardial segments with normal perfusion by radionuclide methods are viable and do not warrant further viability evaluation. Hypoperfused and dysfunctional myocardium can be scar (metabolically inactive) or hibernating (metabolically active). Hibernating myocardium has the potential to improve function with inotropic stimulation or coronary revascularization.

Myocardial viability can be determined using SPECT $\left({ }^{99 \mathrm{~m}} \mathrm{Tc}\right.$ and $\left.{ }^{201} \mathrm{Tl}\right)$, PET radiotracers $\left({ }^{18} \mathrm{~F}\right.$ FDG), or low-dose dobutamine imaging (presence of inotropic contractile reserve), or late gadolinium enhancement cardiac magnetic resonance imaging (presence of scar). Myocardial uptake and retention of ${ }^{99 \mathrm{~m}} \mathrm{Tc}$ and ${ }^{201} \mathrm{Tl}$ tracers indicate integrity of myocyte cell walls and mitochondrial function $\left({ }^{99 \mathrm{~m}} \mathrm{Tc}\right)$, indicating viability, while ${ }^{18}$ F-FDG uptake reflects myocardial glucose metabolic activity. Typical SPECT imaging protocols include rest ${ }^{99 \mathrm{~m}} \mathrm{Tc}$ or ${ }^{201} \mathrm{Tl}$ perfusion imaging, with added redistribution imaging at 4 and/or 24 hours with ${ }^{201} \mathrm{TI}$ (with or without reinjection of ${ }^{201} \mathrm{Tl}$ ), as described previously. ${ }^{39}$ In addition, evaluation of stress perfusion (ischemia implies viability), nitrate enhanced perfusion, ${ }^{139}$ inotropic contractile reserve with changes in regional/global function with low-dose dobutamine ${ }^{140}$ are important adjuncts to enhance the detection of myocardial viability. When available myocardial metabolic imaging with ${ }^{18} \mathrm{~F}-\mathrm{FDG}$ is a more sensitive means to detect viability. In addition to viability, a multitude of other factors impact clinical outcomes in patients with viable myocardium, including patient comorbidities, target vessel status, time to revascularization, adverse LV remodeling among others. Also, if critical amounts of subendocardium are infarcted, even if perfusion is relatively preserved, regional function may not improve post revascularization. This may explain slightly higher sensitivity of techniques based on perfusion and higher specificity of techniques based on contractile reserve assessment to predict recovery of LV function (see Figure 23). Detailed descriptions of SPECT ${ }^{39}$ and PET viability protocols ${ }^{141}$ have been previously published by ASNC.

Qualitative assessment. Myocardial viability can be qualitatively assessed using several parameters. Normal myocardial uptake of ${ }^{99 \mathrm{~m}} \mathrm{Tc}$ or ${ }^{201} \mathrm{Tl}$ tracers indicates viability. The presence of inducible ischemia with improvement of perfusion from stress to rest indicates viability. Redistribution of ${ }^{201} \mathrm{Tl}$ on 4-hour and 24-hour redistribution images in myocardial segments with severely reduced tracer uptake on rest ${ }^{201} \mathrm{Tl}$ images indicates viability. ${ }^{143}$ Hypoperfused myocardial segments with unequivocal improvement following nitrate administration $^{139}$ or contractile reserve on low-dose dobutamine ${ }^{140}$ are viable. When performing ${ }^{201} \mathrm{Tl}$ stress-redistribution-reinjection protocols or ${ }^{99} \mathrm{~m}$ Tc MPI protocols, it is encouraged to administer 0.4 to $0.8 \mathrm{mg}$ sublingual nitroglycerin prior to reinjection to optimize identification of ischemic viable myocardium. This is particularly relevant to patients with evidence of collaterals by invasive coronary angiography. ${ }^{144}$

Semiquantitative assessment. On rest, reinjection, or redistribution images, myocardial segments with normal perfusion or mild hypoperfusion (score 0 or 1) are viable; moderately hypoperfused segments (scores of 2) represent a combination of viable and nonviable myocardium; and severely hypoperfused segments (scores of $\geq 3$ ) represent nonviable myocardium. Examples of viable and nonviable myocardium are shown in Figures 24 and 25.

Quantitative assessment. Rest ${ }^{99 \mathrm{~m}} \mathrm{Tc}$ or ${ }^{201} \mathrm{Tl}$ as well as redistribution, and reinjection ${ }^{201} \mathrm{Tl}$ images can be evaluated quantitatively on polar plots using the following: (1) ischemic-to-normal ratio of myocardial counts also called \% peak radiotracer activity, and (2) defect extent and severity compared to sex matched normal limits databases. ${ }^{143}$ Myocardial segments with $\%$ peak radiotracer activity on normalized polar plots less than or equal to $50 \%$ are considered nonviable and greater than $50 \%$ are considered viable. Pixels of perfusion defect with counts $>2$ standard deviations below gender matched normal limits database are considered nonviable. ${ }^{145}$ Notably, the extent of viability (greater than $10 \%$ hibernating myocardium) ${ }^{146}$ is an important determinant of recovery of $\mathrm{LV}$ function 
Table 8. Myocardial perfusion SPECT: Guidelines for interpretation of rest and stress images

Recommendation

\section{Display medium}

Computer screen meeting ACR-AAPM-SIIM technical standard*

Film/hard copy

\section{Format}

Conventional slice display

Frame normalization

Series normalization

3D display

\section{Review for technical sources of error}

Motion

Attenuation

Reconstruction artifacts

Myocardial count statistics

\section{Initial image interpretation}

Ventricular dilation

Qualitative

Quantitative

TID

Qualitative

Quantitative

Lung uptake (stress and rest), particularly for ${ }^{201} \mathrm{Tl}$

Qualitative

Quantitative

Noncardiac

\section{Perfusion defect assessment}

Location (17 segments)

Extent

Qualitative (small, medium, large)

Semiquantitative (small, 1-2 segments; medium 3-4 segments; large $\geq 5$ segments)

Quantitative (small $<10 \%$; medium $10-20 \%$; large $\geq 20 \%$ )

Severity

Qualitative (mild, moderate, severe, absent tracer uptake)

Semiquantitative (score $0=$ normal; mild or score $1=10 \%$ to $<25 \%$ count reduction; moderate or score $2=25 \%$ to $<50 \%$ count reduction; severe or score $3=\geq 50 \%$

reduction in counts; severe or score 4 (background counts)

Quantitative (computer generated scores)

Reversibility (fixed = no reversibility; mildly reversible; moderately reversible; predominantly reversible; predominantly fixed)

Quantitative (summed stress score, SSS, summed rest score, SRS, summed difference score, SDS)

Quantitative (\% myocardium abnormal, ischemic, scarred)

\section{Gated SPECT}

Display

Quality Control

Regional Wall Motion

Regional wall Thickening

LVEF-Post-stress or rest

Required

Unacceptable

Required

Optional

Required

Optional

Required

Required

Required

Required

Required

Required

Required

Recommended

Recommended Optional

Recommended

Required

Required

Optional

Required

Required

Optional

Required

Recommended

Required

Recommended

Recommended

Required

Required

Required

Required

Required

Required 
Table 8 continued

Recommendation

\section{LV volume}

Qualitative

Quantitative (LV volumes $\mathrm{mL}$ )

Integration of perfusion and function

Normal, probably normal, probably abnormal, abnormal, equivocal

Integration of clinical data with perfusion and function

Infarction, ischemia, peri-infarct ischemia, uninterpretable

Optional

Optional

Required

Required

Required

Required

*ACR-AAPM-SIIM TECHNICAL STANDARD FOR ELECTRONIC PRACTICE OF MEDICAL IMAGING. https://www.acr.org/-/media/ ACR/Files/Practice-Parameters/elec-practice-medimag.pdf

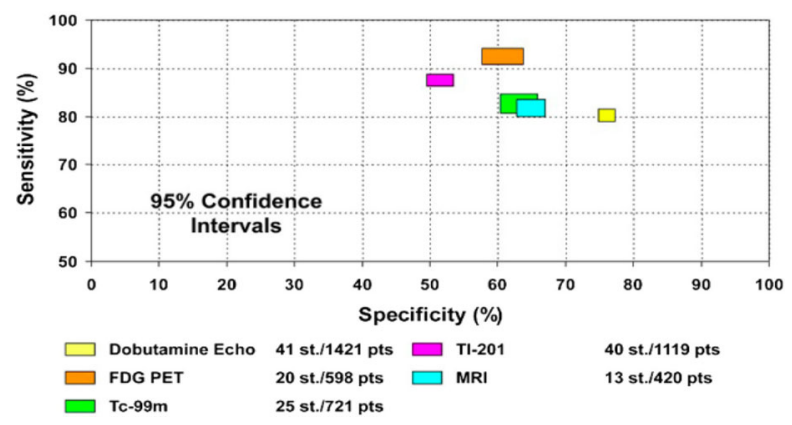

Figure 23. Comparison of sensitivity and specificity of various imaging modalities for detection of viable myocardium. Reproduced with permission from Schinkel AF, et al. Curr Probl Cardiol 2007;32:375-410. ${ }^{142}$

following coronary revascularization. ${ }^{147}$ Table 9 lists the threshold values and the physiological basis for determination of myocardial viability using clinically used radionuclides.

Clinical utility of viability imaging. Although numerous observational studies have shown that revascularization of hibernating myocardium improves survival, this has not been confirmed in randomized trials (i.e., PARR-2, ${ }^{149}$ HEART, ${ }^{150}$ and STICH ${ }^{151}$ ). The Surgical Treatment for Ischemic Heart Failure (STICH) was a randomized trial that tested the hypothesis that surgical revascularization with optimal medical therapy compared to optimal medical therapy alone will improve all-cause mortality. In this randomized study of 1212 patients with CAD amenable to CABG and LVEF less than or equal to $35 \%$ to optimal medical therapy with or without $\mathrm{CABG},{ }^{151}$ although the primary endpoint of allcause mortality did not improve with CABG at 56 months, survival improved at 9.8 years of follow-up (in the STICH Extension Study). ${ }^{152}$ A substudy of STICH evaluated myocardial viability in 601 patients with either dobutamine echo or ${ }^{201}$ Tl SPECT to test the hypothesis that the presence of myocardial viability alters outcomes with CABG. After adjusting for baseline and clinical variables the presence of viability by SPECT or dobutamine stress echocardiography was not associated with improved outcomes. ${ }^{153}$ However, these results are limited for several reasons: lack of randomization in the viability substudy, less frequent co-morbidities in the cohort (low rates of renal dysfunction and heart failure), worse LVEF and remodeled large ventricles in majority of patients who underwent viability testing, lack of inducible ischemia as a criterion for viability, variable ${ }^{201} \mathrm{Tl}$ protocols, and lack of advanced imaging including PET and MRI. Thus, the current evidence does not support withholding revascularization based solely on the results of the imaging tests. Viability imaging is indicated in heart failure and CAD, as well as before revascularization in heart failure patients with $\mathrm{CAD}$ (Class IIa). ${ }^{154}$

\section{Recommendations for Interpretation of SPECT MPI}

- ASNC recommends a systematic and comprehensive approach to interpretation of SPECT MPI.

- Semiquantitative and quantitative interpretation of perfusion images and localization of defects to coronary distribution are recommended.

- LVEF should be quantified.

- Perfusion imaging data should be integrated with clinical and stress testing data, and compared to prior SPECT MPI when applicable.

- Discordant findings should be carefully reviewed and further management suggested if indicated.

- A systematic approach as described previously is critical to ensure accurate and reproducible interpretation of MPI. 


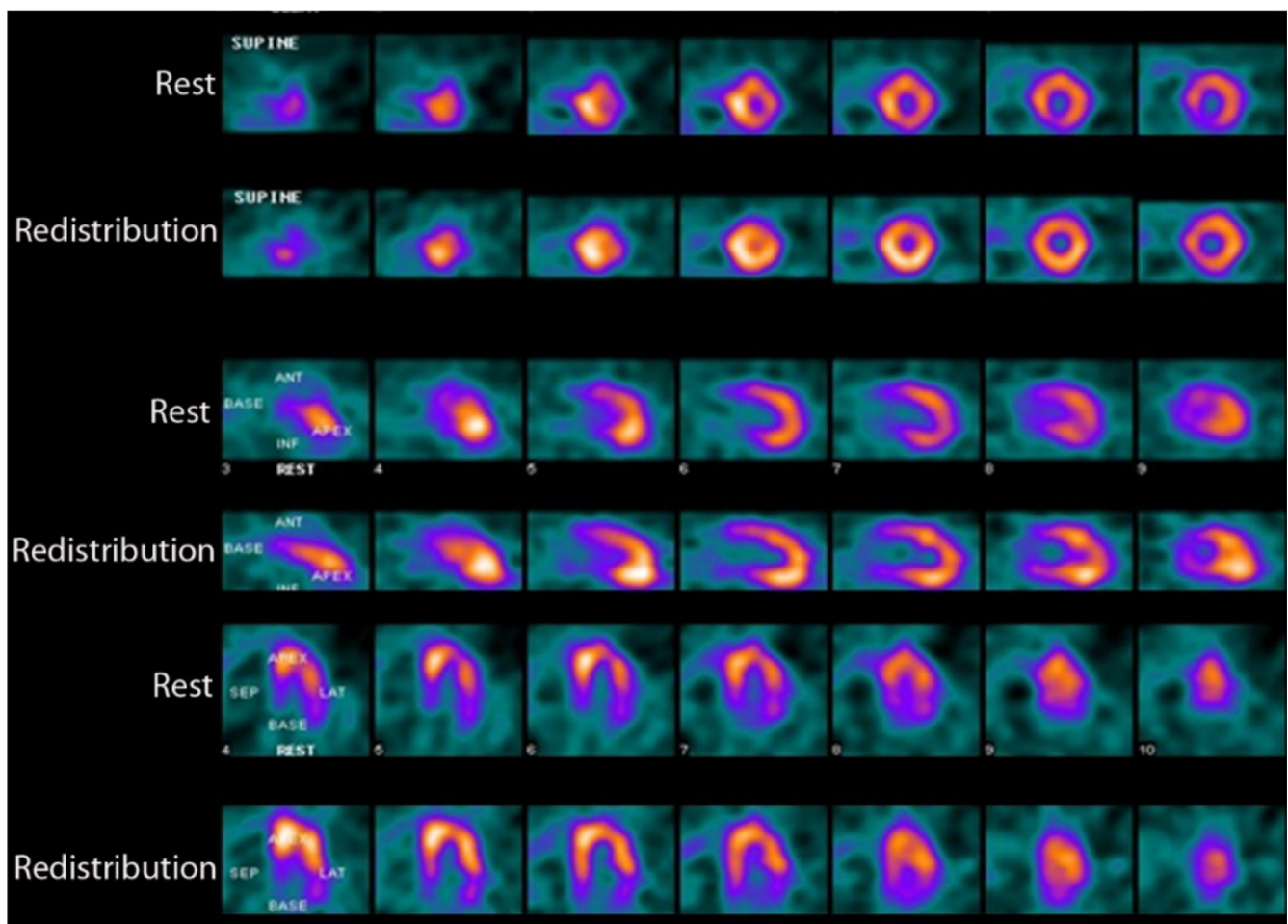

Figure 24. Rest-redistribution ${ }^{201} \mathrm{Tl}$ study for myocardial viability assessment. This image is from a 70-year-old woman with late presentation of inferior ST-elevation myocardial infarction. Electrocardiogram showed inferior $Q$ waves and echocardiogram showed inferior akinesis. Coronary angiography showed occluded right coronary artery with left to right collaterals and $100 \%$ occluded obtuse marginal branch. Rest redistribution nitrate enhanced reinjection 201Tl was ordered. The rest images show moderate decreased perfusion in the basal-mid inferior and inferoseptal walls which showed redistribution on the 4-hour images suggesting the presence of viable myocardium Patient underwent percutaneous revascularization of the right coronary artery.

\section{SPECT MYOCARDIAL BLOOD FLOW QUANTITATION}

\section{Introduction}

Dynamic SPECT images can be obtained with a rapidly rotating conventional SPECT camera, but the development of non-rotating cardiac systems has made the acquisition of dynamic SPECT simpler and more practical. This has led to renewed interest in using SPECT to measure absolute MBF in units of $\mathrm{ml} / \mathrm{min} / \mathrm{g}$ and MFR, the ratio of MBF at stress to MBF at rest. The feasibility of this approach has been demonstrated in a large animal model, ${ }^{155}$ but at this time SPECT MBF and MFR measurements in humans are very new. While the results have been very encouraging, investigations with both conventional rotating systems ${ }^{156,157}$ and non-rotating cardiac cameras ${ }^{15,17,158,159}$ have been limited to single-center feasibility studies. While the benefits of PET-based MBF/MFR are clear, ${ }^{160-163}$ no published study has currently evaluated the incremental diagnostic and prognostic value of SPECT MBF and MFR measurements; more investigations are ongoing in this area.

\section{Acquisition}

Kinetic analysis for MBF requires a set of dynamic SPECT images. A dynamic acquisition will be a major modification from the current standard SPECT acquisition protocol, and the standard perfusion scan will have to be acquired later. Furthermore, it will limit stress testing to pharmacological methods. At present, there is no consensus regarding the acquisition protocol needed for SPECT MBF. Similar to PET protocols for MBF, ${ }^{140}$ the initial temporal sampling of the dynamic series should be rapid (3 to 10 seconds) to allow an accurate image-based measurement of the arterial input function (AIF). Acquisition should begin immediately before injection to ensure that the entire AIF is captured. The length of the period of rapid sampling will depend on the duration of tracer infusion (rapid versus extended bolus). 


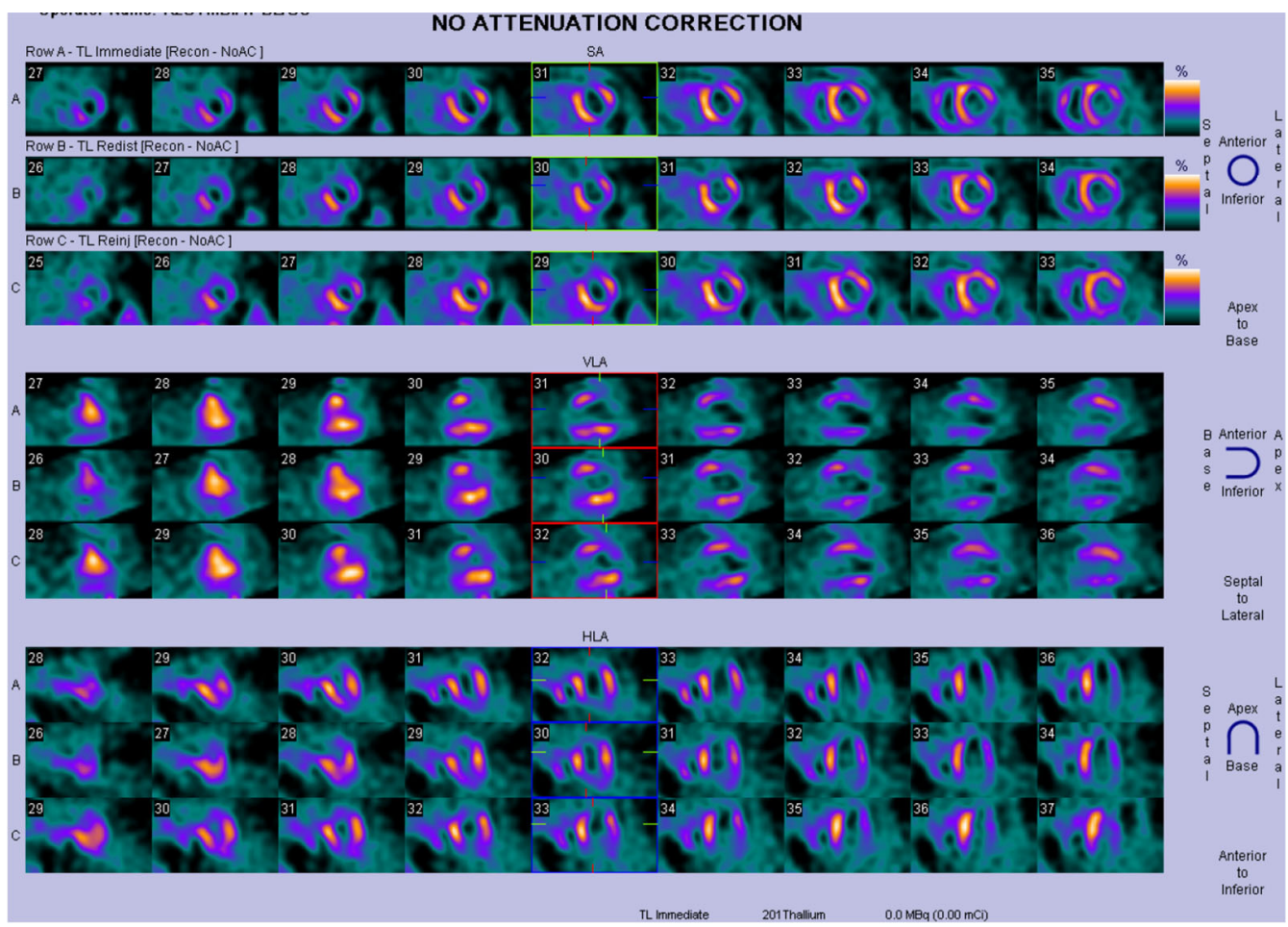

Figure 25. Rest-redistribution ${ }^{201} \mathrm{Tl}$ study for myocardial viability assessment. These images are from an 80-year-old man with known three-vessel coronary disease prior anteroseptal myocardial infarction and three-vessel coronary artery bypass surgery who presents with worsening heart failure symptoms. Echocardiography shows akinetic apical and lateral wall and EF $33 \%$. After medical optimization, he undergoes a pharmacologic stress-redistribution reinjection ${ }^{201}$ TI protocol (sublingual nitroglycerin $0.4 \mathrm{mg}$ administered prior to ${ }^{201} \mathrm{TI}$ reinjection) to assess for ischemia and myocardial viability. The images show rest perfusion defects that did not redistribute consistent with transmural infarction in the mid-distal left anterior descending territory and non-transmural infarction in the left circumflex territory with no significant ischemia. Patient underwent implantable-cardioverter-defibrillator implantation.

A small fraction of the dose may be injected prior to dynamic imaging to aid in positioning the patient in the camera. Following the initial set of rapid images, the duration of the time frames may be increased to reduce noise in the images of the myocardium. The total acquisition duration used has been between 6 and 11 minutes, depending on the imaging system and injected activity. Although PET studies may use the late portion of the dynamic acquisition series for relative MPI, one study has suggested that SPECT images acquired at 45minutes post-injection are superior to images acquired 8 to 12 minutes after injection, ${ }^{164}$ so additional delayed static images are recommended for standard MPI evaluation (Appendix Tables A4-A6).

\section{Processing}

One-tissue-compartment models have been used successfully for sestamibi, tetrofosmin, and ${ }^{201} \mathrm{Tl}$. The uptake rate constant (K1) determined with this model must be corrected for the flow-dependent extraction fraction to obtain the MBF. The extraction-fraction functions across a wide range of MBF values for humans are not well established. Instead, functions derived from animal studies have been used, but the accuracy of this approach in humans has not been established. The rateconstant $\mathrm{K} 1$ is monotonically related to $\mathrm{MBF}$ and so can be used as a surrogate for MBF in the absence of an extraction-fraction correction. However, there is a strong non-linear reduction in K1 as MBF increases, particularly for the ${ }^{99 \mathrm{~m}} \mathrm{Tc}$-based tracers. Consequently, care should be taken in interpreting the K1 values at peak stress or the K1 ratio (rest/stress) as a surrogate MFR. In addition, the $\mathrm{K} 1$ value will depend on the image processing (e.g., attenuation or scatter corrections), as well as the tracer employed. Finally, the reproducibility of SPECT MBF has not been assessed, and thresholds for normal MBF and MPR have not been established. As 
Table 9. Radionuclide imaging methods for myocardial viability

\begin{tabular}{lll}
\hline Radiotracer & \multicolumn{1}{c}{ Characteristic } & \multicolumn{1}{c}{ Viability marker } \\
\hline${ }^{201} \mathrm{TI}$ & Perfusion, cell membrane integrity & $\begin{array}{l}\text { Tracer activity }>50 \% \\
\text { Redistribution }>10 \%\end{array}$ \\
${ }^{99 \mathrm{~m}} \mathrm{TC}$ & Perfusion, cell membrane integrity, intact mitochondria & $\begin{array}{l}\text { Tracer activity }>50 \% \\
\text { Inducible ischemia }\end{array}$ \\
& & Perfusion metabolism mismatch \\
${ }^{18} \mathrm{~F}-\mathrm{FDG}$ & Glucose utilization & Tracer activity $>50 \%$
\end{tabular}

FDG, flourine ${ }^{18}$-deoxyglucose; ${ }^{99 \mathrm{~m}} \mathrm{Tc},{ }^{99}$ technetium; ${ }^{201} \mathrm{TI},{ }^{201}$ thallium

Adapted from Bax JJ, et al. Heart 2004;90 (Suppl V):v26-v33. ${ }^{148}$

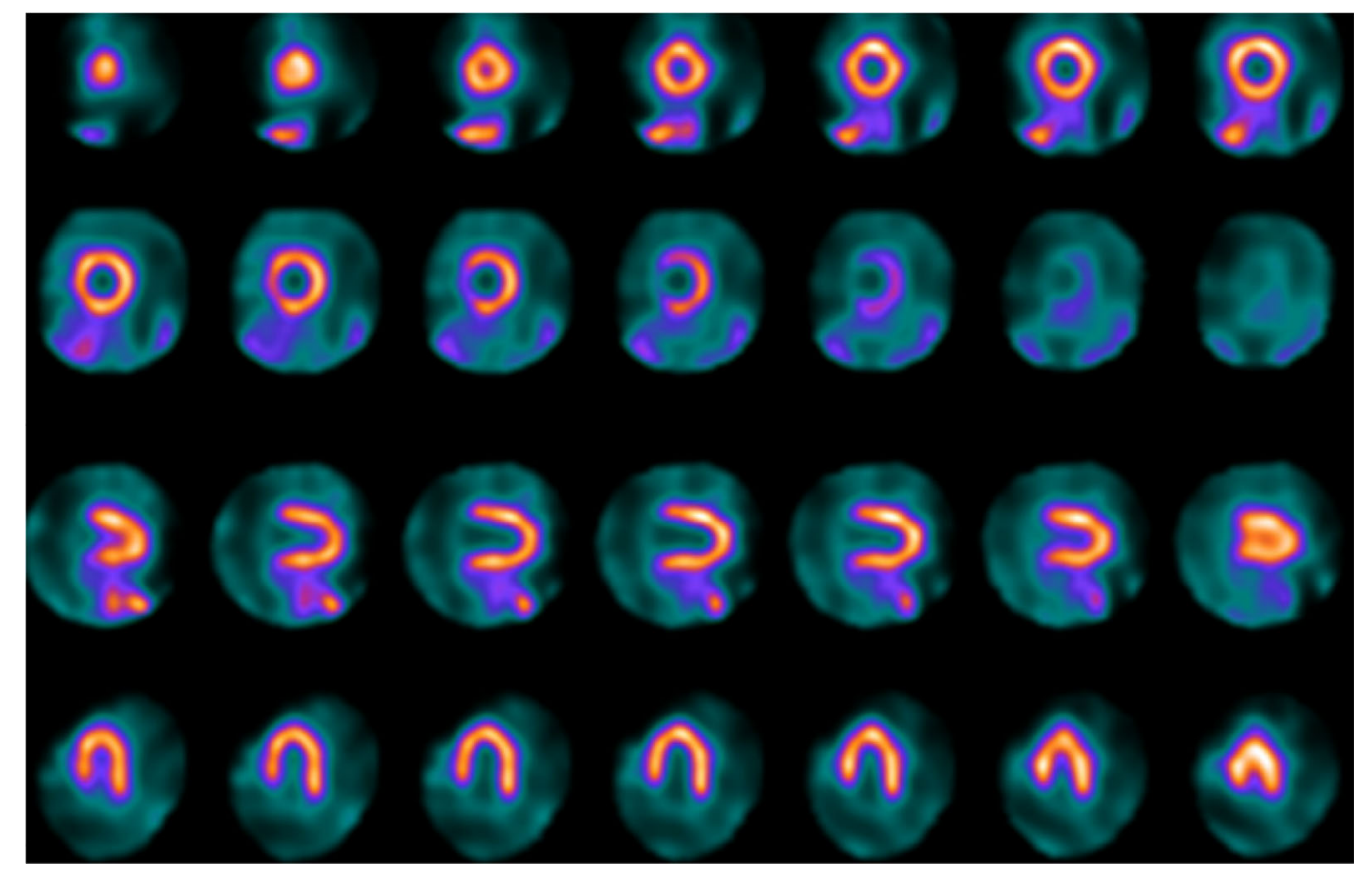

Figure 26. Normal stress-first imaging with upright imaging (CZT camera), avoiding the need for rest imaging.

such, further studies still need to be conducted to validate these measurements before their clinical use.

\section{STRESS-FIRST/STRESS-ONLY IMAGING}

\section{Utility of Stress-First / Stress-Only Imaging}

Stress-only imaging significantly reduces patient radiation exposure (25\% to $80 \%$ ), improves patient convenience, and results in lower cost by eliminating the second radiotracer administration and scan. ${ }^{37,165}$ Reductions to $1 \mathrm{mSv}$ effective dose $(E)$ can be achieved when low-dose stress-only imaging is performed with CZT SPECT cameras (Figure 26), ${ }^{38,166}$ and this approach can contribute to a dramatic reduction in the mean and median nuclear laboratory radiotracer dose. ${ }^{167}$ Stressonly imaging also significantly reduces radiation exposure to nuclear medicine technologists and nursing staff working in nuclear cardiology laboratories ( $\sim 40 \%$ to $50 \%$ ), particularly when combined with CZT imaging. ${ }^{168}$ The safety of performing stress-only imaging has been demonstrated in several large clinical trials which have shown comparably low event rates, whether the study was interpreted as normal based on the stress images alone or the stress/rest comparison. ${ }^{37,165} \mathrm{~A}$ recent randomized study also showed that incorporation of a stress-only imaging algorithm in low- to intermediate-risk patients with acute chest pain was comparable to CT coronary angiography for predicting patient outcome with similar time to diagnosis, hospital stay 


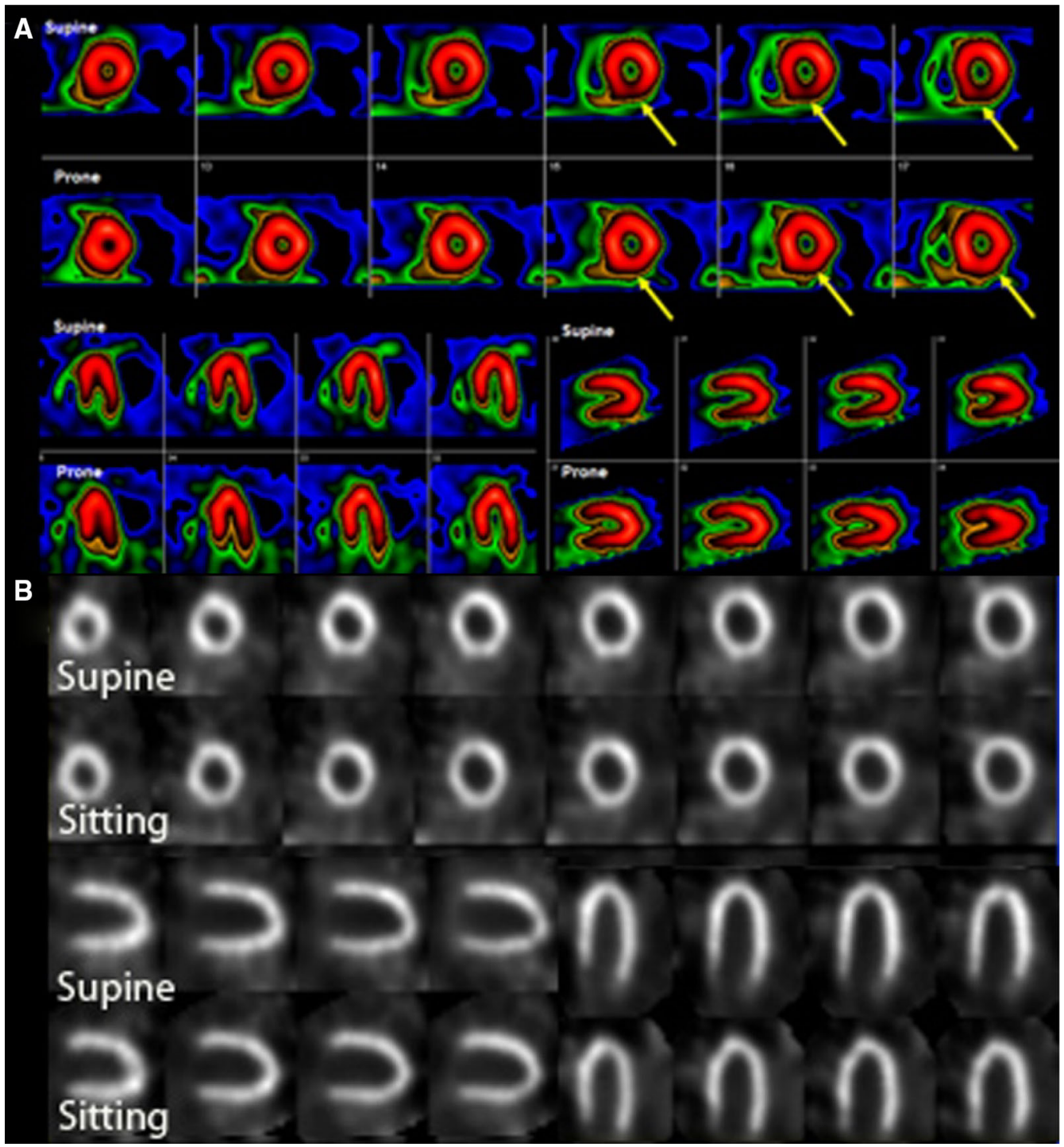

Figure 27. Stress-first imaging with an inferior wall perfusion defect on supine imaging that improved with prone imaging (A, conventional camera) or with upright imaging (B, CZT camera), avoiding the need for rest imaging.

and hospital costs. ${ }^{169}$ Adoption of a stress-rest over a rest-stress imaging protocol is further justified based on temporal trends indicating that most patients with suspected or known CAD (without prior MI) referred for imaging will have a normal stress MPI. ${ }^{170,171}$ Stressonly imaging has been previously endorsed by ASNC. ${ }^{172}$ Of note, AC was performed in most series that showed the efficacy of low dose stress-only SPECT imaging, and the use of AC, upright/supine or supine/ prone imaging (Figure 27), advanced post processing, or advanced camera designs substantially increases the number of patients that can be imaged stress-only. ${ }^{46,173}$

Another argument for stress-first / stress-only imaging comes from an unexpected finding from the recently published EXERRT study. ${ }^{174}$ In particular, the results of the EXERRT study imply for the first time that a sameday rest-stress protocol may not be optimal for identification of regadenoson-induced perfusion defects and ischemia. ${ }^{174}$ This randomized study of 1073 patients (efficacy data set) assessed the safety and accuracy of administering regadenoson early (i.e., 3 minutes) into the slow-walk recovery phase of a submaximal exercise treadmill test versus 1 hour after termination of exercise. An intriguing finding was that about $24 \%$ fewer scans had two or more reversible perfusion defects on the first regadenoson stress SPECT (generally a one-day reststress study) versus the second stress-first SPECT image data set. ${ }^{174}$ The authors commented on this finding and 
suggested that "shine through" of rest activity into the stress study may have reduced the ability to detect perfusion defects and/or ischemia on the initial reststress regadenoson study. ${ }^{167,174}$ A protocol of stress first, with resting images being performed only as necessary, would eliminate the problem of "shine through" on the stress image. ${ }^{175}$

\section{Limitations of Stress-First / Stress-Only SPECT Imaging}

While an approach of low-dose stress-first imaging with provisional-rest-imaging-only-as-needed (PRION) has substantial benefits as outlined previously, and is encouraged, there are certain limitations. For example, a physician reader (or perhaps an advanced technologist) must be available to review the stress images in a timely fashion to carry out a single day protocol. Also, lowdose stress-first acquisitions are best accomplished with advanced SPECT equipment, and can require longer acquisition times. These longer acquisition times can increase rates of patient motion artifact, and require extra vigilance on the part of the technologist and more frequent repeat acquisitions. Also, if soft-tissue attenuation artifacts, or hot-spot artifacts from, for example, LV hypertrophy, are present, it may be impossible to interpret the stress image alone confidently without an accompanying rest acquisition. In such cases, the importance of acquiring a fully diagnostic study takes precedence, and physicians should not hesitate to acquire rest images if they are needed.

\section{Patient Selection for a Stress-First / Stress- Only Imaging Protocol}

Various scoring systems have been proposed regarding proper candidate selection for a stress-first/ stress-only protocol. ${ }^{176,177}$ Despite lack of consensus, one large study enrolling more than 16,000 patients implied that a stress-first study is appropriate in most patients except for those who have known prior myocardial infarction or in those where a two-day protocol is not practical. ${ }^{37}$ In this study by Chang et al, $89 \%$ of patients had either a history of CAD $(31 \%)$ or at least an intermediate risk for CAD $(58 \%) .{ }^{37}$ In addition, $80 \%$ had a BMI greater than 25, and $33 \%$ had a BMI greater than 30 . Body weight was not a criterion for avoiding a stress-first protocol with $38 \%$ of all stressonly patients weighing more than 200 pounds and 53\% of all patients weighing more than 200 pounds having stress-only imaging. ${ }^{37}$ Consistent with these results, a study in 383 patients referred for SPECT prior to bariatric surgery (mean BMI 49) showed that of $89 \%$ who had a normal study, $67 \%$ had stress-only imaging and only $22 \%$ required additional rest imaging. ${ }^{178}$ However, another recent report in more than 18,000 patients indicates that low-dose and ultra, low-dose stress-only protocols are empirically avoided in patients with grade 2 (BMI 35 to $39.9 \mathrm{~kg} / \mathrm{m}$ ) and especially grade 3 (BMI greater than $40 \mathrm{~kg} / \mathrm{m}$ ) obesity. ${ }^{167}$ In such patients, a two-day high-dose imaging protocol is optimal, but the stress study could still be performed first, and if normal, thereby avoid the rest dose. ${ }^{37}$

\section{Criteria for a Normal Stress-First SPECT}

Recent publications have provided a benchmark for what constitutes a normal stress SPECT study. The patient should have homogeneous perfusion throughout the myocardium, normal LV cavity size, a normal LV ejection fraction (greater than 50\%) and normal regional wall motion and LV thickening and normal RV uptake. Interpreting a normal stress-first study with confidence in patients with possible attenuation artifacts can be improved by performing attenuation correction and/or 2position imaging, and this is irrespective of BMI or body habitus. ${ }^{178,179}$ In some cases a normal stress-first MPI study may be accompanied by worrisome exercise treadmill test findings suggestive of ischemia. In such cases, a rest MPI is still not indicated; however, further evaluation with CT angiography (for ischemic ECG changes) or invasive angiography (for high-risk stresstest findings such as ST elevation, exercise induced hypotension, ventricular tachycardia) may be warranted. CT angiography may also be considered for excluding significant CAD in patients with a normal stress-only study but who have ongoing chest pain symptoms of unclear cardiac etiology.

\section{Recommendations for the Use of a Stress- First SPECT}

Laboratories with access to novel technology, software, and AC may consider single day stress-first imaging protocols. With conventional scanners that are not equipped with novel software or $\mathrm{AC}$, consider twoday stress-first protocols to increase the feasibility of stress-first imaging. Advanced post processing and the addition of 2-position imaging can also increase the number of patients successfully imaged stress-only on legacy Anger cameras. ${ }^{180}$ ASNC goal is for laboratories to achieve a median dose of $9 \mathrm{mSv}$ or less for MPI, and stress-first imaging is an important tool for achieving lower radiation doses. 


\section{PATIENT-CENTERED MPI}

\section{Introduction}

In recent years, the medical imaging community, and particularly ASNC, have strongly advocated for tailoring imaging to the individual patient. ${ }^{181-185}$ Although a one-test-fits-all approach may simplify clinical decision-making, it is suboptimal for patient care. The key principals of the desirable individualized imaging approach include justification, that is, appropriate testing, and optimization, i.e., performing the test in an ideal manner. The first principle emphasizes performing the right test for the right patient at the right time. Appropriate Use Criteria can provide guidance in this regard. The second principle incorporates patient comfort and convenience, cost, and especially image quality and radiation dose.

\section{Selection of Imaging Protocol/Scanner}

SPECT imaging protocols and instrumentation are described in prior sections. Imaging protocols should be individually tailored to answer the clinical question with emphasis on image quality and limited radiation exposure. Stress-testing protocols can be tailored to the specific patient and the clinical question (see Table 10). This requires close communication between the referring physician and the imaging team.

\section{Minimizing Radiation Exposure}

Radiation exposure should be minimized by confirming the appropriateness of the radionuclide study, and when feasible, the use of stress only imaging (described previously), weight-based radiotracer dosing, and low-radiotracer-dose imaging.

Minimizing radiotracer dose is desirable in all patients, but more feasible in some than in others. In cooperative patients, the radiotracer dose can be reduced by lengthening acquisition times, whereas in others excessively long acquisitions may be uncomfortable, thereby increasing the likelihood of patient motion and poor image quality. Weight-based, radiotracer-dose algorithms can also reduce radiation dose compared to fixeddose protocols. ${ }^{186}$ The tradeoff between patient dose and acquisition time must be considered on an individual basis taking into account the scanners used. Newer generation imaging equipment, including high-efficiency CZT cameras, ${ }^{187}$ Anger cameras ${ }^{186}$ with improved sensitivity and advanced post-processing solutions-such as iterative reconstruction, resolution recovery, and noise reduction-provide flexible options for reducing radiotracer dose, yet preserving image quality.
The imaging protocol should be adapted to minimize radiation dose in all patients, particularly in young patients, and especially those with low to moderate pretest probability of CAD. In this patient population, it might be particularly attractive to use a stress-first / stressonly imaging protocol in conjunction with lower-dose, full-acquisition time imaging, using a CZT SPECT camera or an Anger camera equipped with advanced post-processing. In other patients, such as those who have back problems or otherwise have a difficult time holding still for imaging, a short acquisition protocol (full dose, more rapid acquisition time) may be a wise option. ${ }^{188}$

\section{Matching the Clinical Question to the Imaging and Stress Testing Protocol}

Answering the clinical question is the priority for any SPECT MPI study. In many patients, the clinical question is straightforward-for example, determining whether the symptoms in a patient with no known CAD are from myocardial ischemia from obstructive epicardial CAD. In other cases, the clinical question may be more complex. For example, a patient with known CAD and prior partial revascularization may have specific coronary lesions on angiography that are of undetermined, but potentially important clinical significance. In such patients, not only are both stress and rest images necessary, but the physician may choose to perform a two-day SPECT imaging study in order to maximize image quality and diagnostic certainty and to lessen the chance that "shinethrough" on a one-day exam confounds the diagnostic accuracy. ${ }^{174}$ The selection of the stress protocol should also be matched to the clinical question.

\section{Combining CT Calcium Score with MPI}

The ACCF/AHA Practice Guidelines for Assessment of Cardiovascular Risk rate CT coronary artery calcium scoring (CACS) as Class IIa for asymptomatic adults at intermediate risk (10\% to $20 \%$ 10-year risk) and Class IIb for individuals at low to intermediate risk (6\% to $10 \%$ 10-year risk). ${ }^{189}$ Many patients referred for MPI have no known CAD and, while not necessarily asymptomatic, are of intermediate pretest likelihood for CAD and meet the Practice Guideline criteria for CACS. With the increasing numbers of SPECT cameras equipped with $\mathrm{CT}$ scanners for use in $\mathrm{AC}$, it is often convenient to perform CACS at the same setting as MPI for patients who have suspected CAD. In patients with a normal stress MPI, the CACS may identify those with calcified atherosclerosis who would likely benefit from aggressive risk factor modification. Coronary artery calcium score frequently changes the behavior of both the treating physician and patient and may aid in MPI 
Table 10. Personalizing stress protocols

\section{Specific patient issues}

Able to exercise adequately

Unable to exercise

Stable angina

Acute chest pain

Troponin elevated

Recent MI ( $<30$ days)

Cardiac catheterization (within 24 hours)

Prior $\mathrm{CABG} / \mathrm{PCI}$

Prior cardiac transplant

Coronary anomaly/dynamic coronary artery compression

Coronary aneurysm

Heart failure

Medication issues

Caffeine intake

Patient on oral dipyridamole

\section{Key issues}

Prefer exercise stress

Pharmacologic stress necessary

Vasodilator stress preferred

Dobutamine stress is an alternative

Prefer exercise stress

Consider testing on anti-ischemic medications if the goal is to determine patient risk on therapy / response to therapy

Consider isotope injection during acute chest pain with rest imaging.

If negative, and unstable angina is not a concern, may proceed with stress testing.

In patients at intermediate to high clinical risk for CAD but low risk for ACS (normal ECG, normal biomarkers and clinically stable) stress-first MPI can be safely performed either with exercise or pharmacologic stress

Evaluate clinically

May proceed with vasodilator stress testing within 24 hours if patient is hemodynamically and clinically stable

Consider only if the patient is clinically and hemodynamically stable

If pre-discharge functional stress testing is desired, consider modified Bruce treadmill test without imaging

If testing is needed to evaluate for hemodynamic significance of residual CAD, proceed with adenosine stress testing If vasodilator stress testing is contraindicated, consider postponing the study for 4 weeks.

Pharmacologic /vasodilator stress is preferred if the arterial access was femoral

Prefer exercise stress

Stress-first imaging can be considered if there is no prior history of $\mathrm{MI}$

Consider vasodilator PET MPI with flow quantitation if available Prefer exercise stress

Dobutamine stress is an alternative if the patient is unable to exercise

Avoid vasodilator stress

Prefer exercise stress

Vasodilator stress is an alternative

Prefer exercise stress

Vasodilator stress is an alternative

Assure that the patient is able to lie supine (for supine scanners)

Prefer upright scanners

If caffeine is consumed within 12 hours, avoid adenosine, dipyridamole, and regadenoson

Consider exercise, dobutamine, or rescheduling the test.

IV adenosine and regadenoson are contraindicated within 48 hours of dipyridamole administration

Consider exercise, dobutamine, or IV dipyridamole 
Table 10 continued

\section{Specific patient issues}

Co-morbidities

End-stage renal disease

Dialysis

Lung disease/COPD

History of seizures

Recent stroke or TIA

Aortic stenosis

Abdominal aortic aneurysm

Peripheral vascular disease

Pre-organ transplant

Preoperative patient

Atrial fibrillation

Left bundle branch block/paced/

ventricular preexcitation

\section{Key issues}

Prefer exercise stress

Vasodilator stress is an alternative

Regadenoson was safe and efficacious in Stage 3-4 ESRD from

one randomized prospective trial

Exercise preferred

Vasodilator an alternate (adenosine, dipyridamole, or

regadenoson)

Pharmacologic stress with dobutamine or

cautiously with regadenoson in stable patients

Seizures have been reported with dipyridamole, adenosine, and regadenoson.

Consider CT coronary angiogram

Avoid vasodilator stress for 2 weeks

Consider CT coronary angiogram

Severe symptomatic AS:

Symptom limited exercise stress is an absolute contraindication

Severe AS without symptoms:

Exercise stress may be considered. Prefer modified Bruce or

Naughton protocols

Vasodilator stress is a relative contraindication

Vasodilator stress preferred

Exercise stress with close monitoring of rate pressure product is an alternative

Vasodilator stress preferred

Vasodilator with exercise is an alternative

Stress testing is considered reasonable in asymptomatic patients with multiple CAD risk factors prior to liver or kidney transplant Exercise stress preferred; aim for age predicted maximal heart rate Use vasodilator stress if adequate exercise stress is not attained Avoid withholding heart rate slowing medications

Prefer vasodilator pharmacologic stress

Exercise stress is relative contraindication in the first week

following cardioversion to sinus rhythm

Diagnostic test (no prior known CAD):

Prefer vasodilator stress

Avoid exercise stress

Avoid exercise plus vasodilator stress

Prognostic test (known CAD): Exercise stress is an alternative

$A S$, aortic stenosis; $C A D$, coronary artery disease; $C T$, computed tomography; $C O P D$, chronic obstructive lung disease; $E S R D$, endstage renal disease; ECG, electrocardiogram; $M P I$, myocardial perfusion imaging; MI, myocardial infarction; TIA, transient ischemia attack

interpretation. $^{190,191}$ CACS also provides incremental prognostic value to MPI. ${ }^{192-194}$ Thus, in patients with no known CAD but an intermediate pretest likelihood, the addition of CACS to a scheduled MPI examination seems reasonable providing the following: the additional information is likely to be used, and the incremental cost and radiation dose is modest. Chest $\mathrm{CT}$ scans performed purely for AC are not of standard quality for calculation of CACS. However, these images still contain diagnostic information relative to the presence and extent of coronary atherosclerosis and other diagnoses, and interpreting physician should inspect these images and integrate this information into the final study report. ${ }^{195-197}$ 


\section{Recommendations for Patient-Centered MPI}

- ASNC strongly endorses a patient-centered imaging approach to MPI to enhance patient satisfaction and improve patient care. Even though at times challenging, a patient-centered approach can minimize test duration, reduce radiation exposure, improve image quality, and optimize the diagnostic accuracy of SPECT MPI.

\section{Acknowledgments}

We would like to thank the reviewers of this document for their input, which has significantly improved the quality of this document. We are grateful to Ms. Victoria Anderson for her organizational assistance and for her expert editorial review of this document.

\section{Disclosures}

Dr. Sharmila Dorbala has received grant support from Astellas Pharma and had investments in General Electric (divested Dec 2017). Dr. Karthik Ananthasubramaniam receives research grant support from Astellas Pharma, GE Healthcare, Alnylam Pharmaceuticals, and serves on the advisory board of Astellas Pharma. Dr. Ian Armstrong received sponsorship from Siemens Healthcare for a PhD project. Dr. Panithaya Chareonthaitawee receives royalties from UpToDate. Dr. Andrew Einstein receives grant support from Toshiba America Medical Systems and has served as a consultant to GE Healthcare. Dr. John Mahmarian serves on the speakers' bureau for Astellas Pharma. Dr. Raymond Russell discloses his spouse is employed by Novartis Institute for Biomedical Research. Dr. Piotr Slomka receives a share of royalties from Cedars-Sinai Medical Center for quantitative MPI algorithms, and receives grant support from them as well as Siemens Medical Systems. Dr. Glenn Wells receives honoraria from GE Healthcare and grant support from GE Healthcare and Advanced Accelerator Applications. All other contributors have nothing relevant to disclose.

\section{APPENDIX}

Table A1. Patient protocol: Same-day stress-rest or rest-stress ${ }^{99 \mathrm{~m}} \mathrm{Tc}$ acquisition for Anger cameras

\begin{tabular}{|c|c|c|c|}
\hline Parameter & $\begin{array}{c}\text { First study } \\
\text { (rest or stress) }\end{array}$ & $\begin{array}{l}\text { Second study } \\
\text { (stress or rest) }\end{array}$ & \\
\hline Administered activity & $8-12 \mathrm{mCi}$ & $24-36 \mathrm{mCi}$ & Standard \\
\hline \multirow[t]{2}{*}{ Position } & Supine & Supine & Standard \\
\hline & Prone & Prone & Optional \\
\hline \multicolumn{4}{|l|}{ Delay time } \\
\hline \multirow[t]{2}{*}{ Injection $\rightarrow$ imaging } & $30-60$ minutes (rest) & 15-60 minutes (stress) & Standard \\
\hline & 15-60 minutes (stress) & 30-60 minutes (rest) & \\
\hline $\begin{array}{l}1^{\text {st }} \text { study } \rightarrow 2^{\text {nd }} \text { study } \\
\text { Acquisition protocol }\end{array}$ & & 30 minutes to 4 hours & Standard \\
\hline Energy window & $15-20 \%$ symmetric, $140 \mathrm{keV}$ & $15-20 \%$ symmetric, $140 \mathrm{keV}$ & Standard \\
\hline Collimator & LEHR & LEHR & Preferred \\
\hline Orbit & $180^{\circ}\left(45^{\circ} \mathrm{RAO}\right.$ to $\left.45^{\circ} \mathrm{LPO}\right)$ & $180^{\circ}\left(45^{\circ} \mathrm{RAO}\right.$ to $\left.45^{\circ} \mathrm{LPO}\right)$ & Preferred \\
\hline \multirow[t]{2}{*}{ Orbit type } & Circular & Circular & Standard \\
\hline & Noncircular & Noncircular & Preferred \\
\hline Pixel size & $3-6 \mathrm{~mm}$ & $3-6 \mathrm{~mm}$ & Standard \\
\hline \multirow[t]{2}{*}{ Acquisition type } & Step and shoot & Step and shoot & Standard \\
\hline & Continuous & Continuous & Optional \\
\hline $\begin{array}{l}\text { Number of } \\
\text { projections }\end{array}$ & $60-64$ & $60-64$ & Standard \\
\hline \multirow[t]{2}{*}{ Matrix } & $64 \times 64$ & $64 \times 64$ & Minimum \\
\hline & $128 \times 128$ & $128 \times 128$ & Preferred \\
\hline Time/projection & $25 \mathrm{~s}$ & $20 \mathrm{~s}$ & Standard \\
\hline ECG gated & Standard & Standard & Preferred \\
\hline
\end{tabular}


Table A1 continued

\begin{tabular}{llll}
\hline Parameter & $\begin{array}{l}\text { First study } \\
\text { (rest or stress) }\end{array}$ & $\begin{array}{l}\text { Second study } \\
\text { (stress or rest) }\end{array}$ & \\
\hline Frames/cycle & 8 & 8 & Standard \\
& 16 & 16 & Preferred \\
R-to-R window & $20-100 \%$ & $20-100 \%$ & $\begin{array}{l}20 \% \text { is recommended, } \\
\text { if extra-frame is provided } \\
\end{array}$ \\
& & & for rejected counts
\end{tabular}

$R A O$, Right anterior oblique; $L P O$, left posterior oblique

Table A2. Patient protocol: Two-day stress-rest ${ }^{99 \mathrm{~m}} \mathrm{Tc}$ acquisition for Anger cameras

\begin{tabular}{|c|c|c|c|}
\hline Parameter & Stress & Rest & \\
\hline \multirow[t]{2}{*}{ Administered activity } & $18-30 \mathrm{mCi}$ & $18-30 \mathrm{mCi}$ & $\mathrm{BMI} \geq 35 \mathrm{~kg} / \mathrm{m}^{2}$ \\
\hline & $8-12 \mathrm{mCi}$ & $8-12 \mathrm{mCi}$ & $\mathrm{BMI}<35 \mathrm{~kg} / \mathrm{m}^{2}$ \\
\hline \multirow[t]{2}{*}{ Position } & Supine & Supine & Standard \\
\hline & Prone & Prone & Optional \\
\hline \multicolumn{4}{|l|}{ Delay time } \\
\hline \multicolumn{3}{|l|}{ Acquisition protocol } & Standard \\
\hline Energy window & $15-20 \%$ symmetric & $15-20 \%$ symmetric & Standard \\
\hline Collimator & LEHR & LEHR & Preferred \\
\hline Orbit & $180^{\circ}\left(45^{\circ} \mathrm{RAO}\right.$ to $\left.45^{\circ} \mathrm{LPO}\right)$ & $180^{\circ}\left(45^{\circ} \mathrm{RAO}\right.$ to $\left.45^{\circ} \mathrm{LPO}\right)$ & Preferred \\
\hline \multirow[t]{2}{*}{ Orbit type } & Circular & Circular & Standard \\
\hline & Noncircular & Noncircular & Standard \\
\hline Pixel size & $3-6 \mathrm{~mm}$ & $3-6 \mathrm{~mm}$ & Standard \\
\hline \multirow[t]{2}{*}{ Acquisition type } & Step and shoot & Step and shoot & Standard \\
\hline & Continuous & Continuous & Optional \\
\hline Number of projections & $60-64$ & $60-64$ & Standard \\
\hline \multirow[t]{2}{*}{ Matrix } & $64 \times 64$ & $64 \times 64$ & Minimum \\
\hline & $128 \times 128$ & $128 \times 128$ & Preferred \\
\hline Time/projection & $25 \mathrm{~s}$ & $20 \mathrm{~s}$ & Standard \\
\hline ECG gated & Standard & Optional & Preferred \\
\hline \multirow[t]{2}{*}{ Frames/cycle } & 8 & 8 & Standard \\
\hline & 16 & 16 & Standard \\
\hline R-to-R window & $100 \%$ & $100 \%$ & Preferred \\
\hline
\end{tabular}

$R A O$, Right anterior oblique; $L P O$, left posterior oblique 
Table A3. Patient protocol: Stress/reinjection*/redistribution ${ }^{201} \mathrm{Tl}$ acquisition for Anger cameras

\begin{tabular}{|c|c|c|c|}
\hline Parameter & Stress/Redistribution & Rest/Reinjection & \\
\hline $\begin{array}{l}\text { Administered activity } \\
\text { Reinjection }\end{array}$ & $2.5-3.5 \mathrm{mCi}$ & $\begin{array}{l}\text { None } \\
1.0 \mathrm{mCi}^{*}\end{array}$ & $\begin{array}{l}\text { Standard } \\
\text { Optional }\end{array}$ \\
\hline Position & $\begin{array}{l}\text { Supine } \\
\text { Prone } \\
\text { Upright/Semiupright }\end{array}$ & $\begin{array}{l}\text { Supine } \\
\text { Prone } \\
\text { Upright/Semiupright }\end{array}$ & $\begin{array}{l}\text { Standard } \\
\text { Optional } \\
\text { Optional }\end{array}$ \\
\hline \multicolumn{4}{|l|}{ Delay time } \\
\hline Injection $\rightarrow$ stress & 10-15 minutes & & Standard \\
\hline Stress $\rightarrow$ rest & & 3-4 hours & Standard \\
\hline \multicolumn{4}{|l|}{ Acquisition protocol } \\
\hline Energy window & $30 \%$ symmetric, $70 \mathrm{keV}$ & $30 \%$ symmetric, $70 \mathrm{keV}$ & Standard \\
\hline Collimator & LEAP & LEAP & Preferred \\
\hline Orbit & $180^{\circ}\left(45^{\circ} \mathrm{RAO}\right.$ to $\left.45^{\circ} \mathrm{LPO}\right)$ & $180^{\circ}\left(45^{\circ} \mathrm{RAO}\right.$ to $\left.45^{\circ} \mathrm{LPO}\right)$ & Preferred \\
\hline Orbit type & $\begin{array}{l}\text { Circular } \\
\text { Noncircular }\end{array}$ & $\begin{array}{l}\text { Circular } \\
\text { Noncircular }\end{array}$ & $\begin{array}{l}\text { Standard } \\
\text { Standard }\end{array}$ \\
\hline Pixel size & $6.4 \pm 0.4 \mathrm{~mm}$ & $6.4 \pm 0.4 \mathrm{~mm}$ & Standard \\
\hline Acquisition type & $\begin{array}{l}\text { Step and shoot } \\
\text { Continuous }\end{array}$ & $\begin{array}{l}\text { Step and shoot } \\
\text { Continuous }\end{array}$ & $\begin{array}{l}\text { Standard } \\
\text { Optional }\end{array}$ \\
\hline Number of projections & $32-64$ & $32-64$ & Standard \\
\hline Matrix & $64 \times 64$ & $64 \times 64$ & Standard \\
\hline Time/projection & $40 \mathrm{~s}$ (32 frame) $25 \mathrm{~s}$ (64 frame) & $40 \mathrm{~s}$ (32 frame) $25 \mathrm{~s}$ (64 frame) & Standard \\
\hline ECG gated & Standard & Optional & Preferred \\
\hline \multirow[t]{2}{*}{ Frames/cycle } & 8 & 8 & Standard \\
\hline & 16 & 16 & Standard \\
\hline R-to-R window & $100 \%$ & $100 \%$ & Preferred \\
\hline
\end{tabular}

${ }^{*} \mathrm{~A}$ smaller dose of ${ }^{201} \mathrm{Tl}$ may be injected 20 to 30 minutes prior to rest imaging

$R A O$, Right anterior oblique; $L P O$, left posterior oblique

Table A4. Patient protocol: Same-day stress-rest or rest-stress ${ }^{99 \mathrm{~m}} \mathrm{Tc}$ acquisition for solid-state cadmium-zinc-telluride (CZT) cameras

\begin{tabular}{|c|c|c|c|}
\hline \multirow[b]{2}{*}{ Parameter } & \multicolumn{2}{|c|}{ Rest and Stress } & \\
\hline & $\begin{array}{c}\text { Study } 1 \\
\text { Rest or Stress }\end{array}$ & $\begin{array}{c}\text { Study } 2 \\
\text { Stress or Rest }\end{array}$ & \\
\hline Administered activity & 4-6 mCi & $12-18 \mathrm{mCi}$ & Standard \\
\hline Position* & $\begin{array}{l}\text { Supine/Upright } \\
\text { Prone }\end{array}$ & $\begin{array}{l}\text { Supine/Upright } \\
\text { Prone }\end{array}$ & $\begin{array}{l}\text { Standard } \\
\text { Optional }\end{array}$ \\
\hline \multicolumn{4}{|l|}{ Delay time } \\
\hline Injection $\rightarrow$ imaging & $\begin{array}{l}30-60 \text { minutes (rest) } \\
15-60 \text { minutes (stress) }\end{array}$ & $\begin{array}{l}30-60 \text { minutes (rest) } \\
15-60 \text { minutes (stress) }\end{array}$ & Standard \\
\hline $\begin{array}{l}1^{\text {st }} \text { study } \rightarrow 2^{\text {nd }} \text { study } \\
\text { Acquisition protocol }\end{array}$ & 30 minutes to 4 hours & 30 minutes to 4 hours & Standard \\
\hline Energy window & $15-20 \%$ symmetric, $140 \mathrm{keV}$ & $15-20 \%$ symmetric, $140 \mathrm{keV}$ & Standard \\
\hline Collimator* & Wide-angle/Multi-pinhole & Wide-angle/Multi-pinhole & Preferred \\
\hline Pixel size & $2-3 \mathrm{~mm}$ & $2-3 \mathrm{~mm}$ & Standard \\
\hline
\end{tabular}


Table A4 continued

\begin{tabular}{llll}
\hline Parameter & Rest and Stress & & \\
\cline { 2 - 3 } & $\begin{array}{l}\text { Study 1 } \\
\text { Rest or Stress }\end{array}$ & $\begin{array}{l}\text { Study 2 } \\
\text { Stress or Rest }\end{array}$ & Standard \\
\hline Acquisition type & Continuous & Continuous & Standard \\
Number of projections* & N/A & N/A & Standard \\
Matrix & $64 \times 64$ (minimum) & $64 \times 64$ (minimum) & Preferred \\
Acquisition time & $128 \times 128$ & $128 \times 128$ & Standard \\
ECG gated & $5-14$ min (1 million counts) & $3-6$ min (1 million counts) & Preferred \\
Frames/cycle & Optional (rest); Standard (stress) & Optional (rest); Standard (stress) & Standard \\
& 8 & 8 & Standard \\
R-to-R window & 16 & 16 & Preferred \\
\hline
\end{tabular}

*Vendor-specific design. $R A O$, Right anterior oblique; $L P O$, left posterior oblique

Table A5. Patient protocol: Two-day stress ${ }^{99 \mathrm{~m}}$ Tc acquisition for solid-state cadmium-zinc-telluride (CZT) cameras

\begin{tabular}{|c|c|c|c|}
\hline Parameter & Stress & Rest & \\
\hline \multirow[t]{2}{*}{ Administered activity } & $4-6 \mathrm{mCi}$ & $4-6 \mathrm{mCi}$ & $\mathrm{BMI}<35 \mathrm{~kg} / \mathrm{m}^{2}$ \\
\hline & $9-15 \mathrm{mCi}$ & $9-15 \mathrm{mCi}$ & $\mathrm{BMI} \geq 35 \mathrm{~kg} / \mathrm{m}^{2}$ \\
\hline \multirow[t]{2}{*}{ Position * } & Supine/Semiupright & Supine/Semiupright & Standard \\
\hline & Prone & Prone & Optional \\
\hline \multicolumn{4}{|l|}{ Delay time } \\
\hline Injection $\rightarrow$ imaging & $15-60$ minutes & $30-60$ minutes & Standard \\
\hline \multicolumn{4}{|l|}{ Acquisition protocol } \\
\hline Energy window & $15-20 \%$ symmetric, $140 \mathrm{keV}$ & $15-20 \%$ symmetric, $140 \mathrm{keV}$ & Standard \\
\hline Collimator* & Wide-angle/Multi-pinhole & Wide-angle/Multi-pinhole & Preferred \\
\hline Pixel size & $2.5 \mathrm{~mm}$ & $2.5 \mathrm{~mm}$ & Standard \\
\hline Acquisition type & Continuous & Continuous & Standard \\
\hline Number of projections* & N/A & N/A & Standard \\
\hline \multirow[t]{2}{*}{ Matrix } & $64 \times 64$ & $64 \times 64$ & Standard \\
\hline & $128 \times 128$ & $128 \times 128$ & Optional \\
\hline Acquisition time & $5-14$ min ( 1 million counts) & $5-14$ min (1 million counts) & Standard \\
\hline ECG gated & Standard & Optional & Preferred \\
\hline \multirow[t]{2}{*}{ Frames/cycle } & 8 & 8 & Standard \\
\hline & 16 & 16 & Standard \\
\hline R-to-R window & $100 \%$ & $100 \%$ & Preferred \\
\hline
\end{tabular}

*Vendor-specific design. RAO, Right anterior oblique; $L P O$, left posterior oblique 
Table A6. Patient protocol: Stress/reinjection*/redistribution ${ }^{201} \mathrm{Tl}$ acquisition for solid-state cadmium-zinc-telluride (CZT) cameras

\begin{tabular}{|c|c|c|c|}
\hline Parameter & Stress/Redistribution & Rest/Reinjection & \\
\hline Administered activity & $1.3-1.8 \mathrm{mCi}$ & None & Standard \\
\hline Reinjection & & $0.5 \mathrm{mCi}^{*}$ & Optional \\
\hline \multirow[t]{2}{*}{ Position $^{\dagger}$} & Supine/Semiupright & Supine/Semiupright & Standard \\
\hline & Prone & Prone & Optional \\
\hline \multicolumn{4}{|l|}{ Delay time } \\
\hline Injection $\rightarrow$ stress & 10-15 minutes & & Standard \\
\hline Stress $\rightarrow$ rest & & 3-4 hours & Standard \\
\hline Reinjection $\rightarrow$ rest & & 20-30 minutes & Optional \\
\hline \multicolumn{4}{|l|}{ Acquisition protocol } \\
\hline Energy window & $30 \%$ symmetric, $70 \mathrm{keV}$ & $30 \%$ symmetric, $70 \mathrm{keV}$ & Standard \\
\hline Collimator $^{\dagger}$ & Wide-angle/Multi-pinhole & Wide-angle/Multi-pinhole & Preferred \\
\hline Pixel size & $2.5 \mathrm{~mm}$ & $2.5 \mathrm{~mm}$ & Standard \\
\hline Acquisition type & Continuous & Continuous & Standard \\
\hline Number of projections $\dagger$ & $19 / 120$ & $19 / 120$ & Standard \\
\hline \multirow[t]{2}{*}{ Matrix } & $64 \times 64$ & $64 \times 64$ & Minimum \\
\hline & $128 \times 128$ & $128 \times 128$ & Preferred \\
\hline Acquisition time & $5-8 \min$ & $5-8 \min$ & Standard \\
\hline ECG gated & Standard & Optional & Preferred \\
\hline \multirow{2}{*}{ Frames/cycle } & 8 & 8 & Standard \\
\hline & 16 & 16 & Standard \\
\hline R-to-R window & $100 \%$ & $100 \%$ & Preferred \\
\hline
\end{tabular}

${ }^{*}$ A smaller dose of ${ }^{201} \mathrm{Tl}$ may be injected 20 to 30 minutes prior to rest imaging. ${ }^{\dagger}$ Vendor-specific design

$R A O$, Right anterior oblique; $L P O$, left posterior oblique

\section{References}

1. Holly TA, Abbott BG, Al-Mallah M, Calnon DA, Cohen MC, DiFilippo FP, et al. ASNC Imaging Guidelines for Nuclear Cardiology Procedures: Single photon-emission computed tomography. J Nucl Cardiol. 2010. https://doi.org/10.1007/ s12350-010-9246-y.

2. Tilkemeier PL, Bourque J, Doukky R, Sanghani R, Weinberg RL. ASNC Imaging Guidelines for Nuclear Cardiology Procedures: Standardized reporting of nuclear cardiology procedures. J Nucl Cardiol. 2017. https://doi.org/10.1007/s12350-017-1057-y.

3. Slomka PJ, Patton JA, Berman DS, Germano G. Advances in technical aspects of myocardial perfusion SPECT imaging. J Nucl Cardiol. 2009;16:255-76.

4. O'Connor MK. Instrument- and computer-related problems and artifacts in nuclear medicine. Semin Nucl Med. 1996;26:256-77.

5. Bai C, Conwell R, Kindem J, Babla H, Gurley M, De Los Santos $\mathrm{R}$, et al. Phantom evaluation of a cardiac SPECT/VCT system that uses a common set of solid-state detectors for both emission and transmission scans. J Nucl Cardiol. 2010;17:459-69. https:// doi.org/10.1007/s12350-010-9204-8.

6. Bocher M, Blevis IM, Tsukerman L, Shrem Y, Kovalski G, Volokh L. A fast cardiac gamma camera with dynamic SPECT capabilities: Design, system validation and future potential. Eur J
Nucl Med Mol Imaging. 2010;37:1887-902. https://doi.org/10. 1007/s00259-010-1488-Z

7. Erlandsson K, Kacperski K, van Gramberg D, Hutton BF. Performance evaluation of D-SPECT: A novel SPECT system for nuclear cardiology. Phys Med Biol. 2009;54:2635-49.

8. Bushberg JT, Seibert JA, Leidholt EM, Boone JM. The essential physics of medical imaging. Baltimore: Williams \& Wilkins; 1994.

9. Cherry SR, Sorenson JA, Phelps ME. Physics in nuclear medicine. 3rd ed. Philadelphia: Elsevier Science; 2003.

10. DiFilippo FP, Abreu SH, Majmundar H. Collimator integrity. J Nucl Cardiol. 2006;13:889-91.

11. Hawman PC, Haines EJ. The cardiofocal collimator: A variablefocus collimator for cardiac SPECT. Phys Med Biol. 1994;39:439-50.

12. Caobelli F, Ren Kaiser S, Thackeray JT, Bengel FM, Chieregato $\mathrm{M}$, Soffientini A, et al. The importance of a correct positioning of the heart using IQ-SPECT system with multifocal collimators in myocardial perfusion imaging: A phantom study. J Nucl Cardiol. 2015;22:57-65.

13. Steele PP, Kirch DL, Koss JE. Comparison of simultaneous dualisotope multipinhole SPECT with rotational SPECT in a group of patients with coronary artery disease. J Nucl Med. 2008;49:1080-9. 
14. Esteves FP, Raggi P, Folks RD, Keidar Z, Askew JW, Rispler S, et al. Novel solid-state-detector dedicated cardiac camera for fast myocardial perfusion imaging: Multicenter comparison with standard dual detector cameras. J Nucl Cardiol. 2009;16:927-34.

15. Ben-Haim S, Murthy VL, Breault C, Allie R, Sitek A, Roth N, et al. Quantification of myocardial perfusion reserve using dynamic SPECT imaging in humans: A feasibility study. J Nucl Med. 2013;54:873-9.

16. Wells RG, Marvin B, Poirier M, Renaud JM, deKemp RA, Ruddy TD. Optimization of SPECT measurement of myocardial blood flow with corrections for attenuation, motion, and bloodbinding compared to PET. J Nucl Med. 2017. https://doi.org/10. 2967/jnumed.117.191049.

17. Nkoulou R, Fuchs TA, Pazhenkottil AP, Kuest SM, Ghadri JR, Stehli J, et al. Absolute myocardial blood flow and flow reserve assessed by gated SPECT with cadmium-zinc-telluride detectors using 99mTc-tetrofosmin: Head to head comparison with $13 \mathrm{~N}$ ammonia PET. J Nucl Med. 2016;57:1887-92.

18. Shin JH, Pokharna HK, Williams KA, Mehta R, Ward RP. SPECT myocardial perfusion imaging with prone-only acquisitions: Correlation with coronary angiography. J Nucl Cardiol. 2009;16:590-6.

19. Nishina H, Slomka PJ, Abidov A, Yoda S, Akincioglu C, Kang $\mathrm{X}$, et al. Combined supine and prone quantitative myocardial perfusion SPECT: Method development and clinical validation in patients with no known coronary artery disease. J Nucl Med. 2006;47:51-8.

20. Nakazato R, Slomka PJ, Fish M, Schwartz RG, Hayes SW, Thomson LE, et al. Quantitative high-efficiency cadmium-zinctelluride SPECT with dedicated parallel-hole collimation system in obese patients: results of a multi-center study. J Nucl Cardiol. 2015;22:266-75. https://doi.org/10.1007/s12350-014-9984-3.

21. Nakazato R, Tamarappoo BK, Kang X, Wolak A, Kite F, Hayes SW, et al. Quantitative upright-supine high-speed SPECT myocardial perfusion imaging for detection of coronary artery disease: correlation with invasive coronary angiography. J Nucl Med. 2010;51:1724-31. https://doi.org/10.2967/jnumed.110. 078782.

22. Schepis T, Gaemperli O, Koepfli P, Ruegg C, Burger C, Leschka $\mathrm{S}$, et al. Use of coronary calcium score scans from stand-alone multislice computed tomography for attenuation correction of myocardial perfusion SPECT. Eur J Nucl Med Mol Imaging. 2007;34:11-9.

23. National Electrical Manufacturers Association. NEMA Standards Publication NU 1-2012: Performance Measurements of Gamma Cameras. Washington, DC: National Electrical Manufacturers Association. Published Sept 5, 2013.

24. Esser PD, Graham LS. A quality control program for nuclear medicine cameras. In: Henkin RE, editor. nuclear medicine. 2nd ed. Philadelphia: Mosby; 2006. p. 246-56.

25. ACR-AAPM Technical Standard for Medical Physics Performance Monitoring of SPECT-CT Equipment. Revised 2014 (Resolution 35). https://www.acr.org/-/media/ACR/Files/ Practice-Parameters/SPECT-CT-Equip.pdf

26. American Society of Nuclear Cardiology. ASNC imaging guidelines for nuclear cardiology procedures: Introduction of new technology for clinical use. J Nucl Cardiol. 2009;16:166.

27. Nichols KG, Galt JR. Quality control for SPECT imaging. In: DePuey EG, Berman DS, Garcia EV, editors. Cardiac SPECT imaging. 2nd ed. Philadelphia: Lippincott Williams \& Wilkins; 2001. p. 17-40.

28. DePuey EG. How to detect and avoid myocardial perfusion SPECT artifacts. J Nucl Med. 1994;35:699-702.
29. Galt JR, Faber T. Principles of single photon emission computed tomography (SPECT) imaging. In: Christian PE, Bernier DR, Langan JK, editors. Nuclear medicine and PET: Technology and techniques. St. Louis: Mosby; 2003. p. 242-84.

30. Cerqueira MD, Matsuoka D, Ritchie JL, Harp GD. The influence of collimators on SPECT center of rotation measurements: Artifact generation and acceptance testing. $\mathrm{J}$ Nucl Med. 1988;29:1393-7.

31. Hines H, Kayayan R, Colsher J, Hashimoto D, Schubert R, Fernando J, et al. National Electrical Manufacturers Association recommendation for implementing SPECT instrumentation quality control. J Nucl Med. 2000;41:383-9.

32. Greer KL, Jaszczak RJ, Coleman RE. An overview of a camerabased SPECT system. Med Phys. 1982;9:455-63.

33. Kennedy JA, Yosilevsky G, Przewloka K, Israel O, Frenkel A. 3D spatial resolution map and sensitivity characterization of a dedicated cardiac CZT SPECT camera. J Nucl Med. 2009;50:107.

34. American College of Radiology. ACR Web site. http://www.acr. org.

35. American Association of Physicists in Medicine. AAPM Web site. http://www.aapm.org.

36. Seo Y, Mari C, Hasegawa BH. Technological development and advances in single-photon emission computed tomography/computed tomography. Semin Nucl Med. 2008;38:177-98. https://doi.org/10.1053/j.semnuclmed.2008.01.001.

37. Chang SM, Nabi F, Xu J, Raza U, Mahmarian JJ. Normal stressonly versus standard stress/rest myocardial perfusion imaging: Similar patient mortality with reduced radiation exposure. J Am Coll Cardiol. 2010;55:221-30. https://doi.org/10.1016/j.jacc. 2009.09.022.

38. Einstein AJ, Johnson LL, DeLuca AJ, Kontak AC, Groves DW, Stant J, et al. Radiation dose and prognosis of ultra-low-dose stress-first myocardial perfusion SPECT in patients with chest pain using a high-efficiency camera. J Nucl Med. 2015;56:54551. https://doi.org/10.2967/jnumed.114.150664.

39. Henzlova MJ, Duvall WL, Einstein AJ, Travin MI, Verberne HJ. ASNC imaging guidelines for SPECT nuclear cardiology procedures: Stress, protocols, and tracers. J Nucl Cardiol. 2016;23:606-39. https://doi.org/10.1007/s12350-015-0387-x.

40. Zeintl J, Vija AH, Yahil A, Hornegger J, Kuwert T. Quantitative accuracy of clinical $99 \mathrm{mTc}$ SPECT/CT using ordered-subset expectation maximization with 3-dimensional resolution recovery, attenuation, and scatter correction. J Nucl Med. 2010;51:921-8. https://doi.org/10.2967/jnumed.109.071571.

41. Garcia EV, Faber TL, Esteves FP. Cardiac dedicated ultrafast SPECT cameras: New designs and clinical implications. J Nucl Med. 2011;52:210-7. https://doi.org/10.2967/jnumed.110. 081323.

42. Lyon MC, Foster C, Ding X, Dorbala S, Spence D, Bhattacharya $\mathrm{M}$, et al. Dose reduction in half-time myocardial perfusion SPECT-CT with multi-focal collimation. J Nucl Cardiol. 2016;23:657-67. https://doi.org/10.1007/s12350-016-0471-x.

43. Nakazato R, Berman DS, Hayes SW, Fish M, Padgett R, Xu Y, et al. Myocardial perfusion imaging with a solid-state camera: Simulation of a very low dose imaging protocol. J Nucl Med. 2013;54:373-9. https://doi.org/10.2967/jnumed.112.110601.

44. Segall GM, Davis MJ. Prone versus supine thallium myocardial SPECT: A method to decrease artifactual inferior wall defects. J Nucl Med. 1989;30:548-55.

45. Kiat H, Van Train KF, Friedman JD, Germano G, Silagan G, Wang FP, et al. Quantitative stress-redistribution thallium-201 SPECT using prone imaging: Methodologic development and validation. J Nucl Med. 1992;33:1509-15. 
46. Guner LA, Caliskan B, Isik I, Aksoy T, Vardareli E, Parspur A. Evaluating the role of routine prone acquisition on visual evaluation of SPECT images. J Nucl Med Technol. 2015;43:282-8. https://doi.org/10.2967/jnmt.115.165936.

47. Taasan V, Wokhlu A, Taasan MV, Dusaj RS, Mehta A, Kraft S, et al. Comparative accuracy of supine-only and combined supineprone myocardial perfusion imaging in men. J Nucl Cardiol. 2016;23:1470-6.

48. Esquerre JP, Coca FJ, Martinez SJ, Guiraud RF. Prone decubitus: A solution to inferior wall attenuation in thallium-201 myocardial tomography. J Nucl Med. 1989;30:398-401.

49. Slomka PJ, Nishina H, Abidov A, Hayes SW, Friedman JD, Berman DS, et al. Combined quantitative supine-prone myocardial perfusion SPECT improves detection of coronary artery disease and normalcy rates in women. $\mathrm{J}$ Nucl Cardiol. 2007; 14:44-52.

50. Ben-Haim S, Almukhailed O, Neill J, Slomka P, Allie R, Shiti D, et al. Clinical value of supine and upright myocardial perfusion imaging in obese patients using the D-SPECT camera. J Nucl Cardiol. 2014;21:478-85. https://doi.org/10.1007/s12350-0149853-0.

51. Hindorf C, Oddstig J, Hedeer F, Hansson MJ, Jogi J, Engblom H. Importance of correct patient positioning in myocardial perfusion SPECT when using a CZT camera. J Nucl Cardiol. 2014;21:695702.

52. Friedman J, Van Train K, Maddahi J, Rozanski A, Prigent F, Bietendorf J, et al. "Upward creep" of the heart: A frequent source of false-positive reversible defects during thallium-201 stress-redistribution SPECT. J Nucl Med. 1989;30:1718-22.

53. Ben-Haim S, Kacperski K, Hain S, Van Gramberg D, Hutton BF, Erlandsson K, et al. Simultaneous dual-radionuclide myocardial perfusion imaging with a solid-state dedicated cardiac camera. Eur J Nucl Med Mol Imaging. 2010;37:1710-21. https://doi.org/ 10.1007/s00259-010-1441-1.

54. Blaire T, Bailliez A, Bouallegue FB, Bellevre D, Agostini D, Manrique A. Left ventricular function assessment using 123I/ 99mTc dual-isotope acquisition with two semi-conductor cadmium-zinc-telluride (CZT) cameras: A gated cardiac phantom study. EJNMMI Physics. 2016;3:27. https://doi.org/10.1186/ s40658-016-0163-2.

55. Patil HR, Bateman TM, McGhie AI, Burgett EV, Courter SA, Case JA, et al. Diagnostic accuracy of high-resolution attenuation-corrected Anger-camera SPECT in the detection of coronary artery disease. J Nucl Cardiol. 2014;21:127-34. https://doi.org/ 10.1007/s12350-013-9817-9.

56. Bateman TM, Berman DS, Heller GV, Brown KA, Cerqueira MD, Verani MS, et al. American Society of Nuclear Cardiology position statement on electrocardiographic gating of myocardial perfusion SPECT scintigrams. J Nucl Cardiol. 1999;6:470-1.

57. Cullom SJ, Case JA, Bateman TM. Electrocardiographically gated myocardial perfusion SPECT: Technical principles and quality control considerations. J Nucl Cardiol. 1998;5:418-25.

58. DePuey EG, Nichols K, Dobrinsky C. Left ventricular ejection fraction assessed from gated technetium-99m-sestamibi SPECT. J Nucl Med. 1993;34:1871-6.

59. Smanio PE, Watson DD, Segalla DL, Vinson EL, Smith WH, Beller GA. Value of gating of technetium-99m sestamibi singlephoton emission computed tomographic imaging. J Am Coll Cardiol. 1997;30:1687-92.

60. Germano G, Berman DS. On the accuracy and reproducibility of quantitative gated myocardial perfusion SPECT. J Nucl Med. 1999;40:810-3.

61. Navare SM, Wackers FJ, Liu YH. Comparison of 16-frame and 8 -frame gated SPET imaging for determination of left ventricular volumes and ejection fraction. Eur J Nucl Med Mol Imaging. 2003;30:1330-3.

62. He ZX, Verani MS, Liu XJ. Nitrate-augmented myocardial imaging for assessment of myocardial viability. J Nucl Cardiol. $1995 ; 2: 352-7$.

63. Hendel RC, Berman DS, Cullom SJ, Follansbee W, Heller GV, Kiat $\mathrm{H}$, et al. Multicenter clinical trial to evaluate the efficacy of correction for photon attenuation and scatter in SPECT myocardial perfusion imaging. Circulation. 1999;99:2742-9.

64. Thompson RC, Heller GV, Johnson LL, Case JA, Cullom SJ, Garcia EV, et al. Value of AC on ECG-gated SPECT myocardial perfusion imaging related to body mass index. J Nucl Cardiol. 2005; 12:195-202

65. Arsanjani R, Xu Y, Hayes SW, Fish M, Lemley M Jr, Gerlach J, et al. Comparison of fully automated computer analysis and visual scoring for detection of coronary artery disease from myocardial perfusion SPECT in a large population. J Nucl Med. 2013;54:221-8. https://doi.org/10.2967/jnumed.112.108969.

66. Hansen CL. Digital image processing for clinicians, part I: Basics of image formation. J Nucl Cardiol. 2002;9:343-9.

67. Hansen CL, Kramer M, Rastogi A. Lower accuracy of Tl-201 SPECT in women is not improved by size-based normal databases or Wiener filtering. J Nucl Cardiol. 1999;6:177-82.

68. King MA, Glick SJ, Penney BC, Schwinger RB, Doherty PW. Interactive visual optimization of SPECT prereconstruction filtering. J Nucl Med. 1987;28:1192-8.

69. Hansen CL. Digital image processing for clinicians, part II: Filtering. J Nucl Cardiol. 2002;9:429-37.

70. Hudson HM, Larkin RS. Accelerated image reconstruction using ordered subsets of projection data. IEEE Trans Med Imaging. 1994;13:601-9.

71. Yester MV. SPECT image reconstruction. In: Henkin RE, editor. Nuclear medicine. 2nd ed. Philadelphia: Mosby; 2006. p. 18595.

72. DePuey EG. Advances in SPECT camera software and hardware (review article). J Nucl Cardiol. 2012;19:551-81.

73. Borges-Neto S, Pagnanelli RA, Shaw LJ, Honeycutt E, Shwartz SC, Adams GL, et al. J Nucl Cardiol. 2007;14:555-65.

74. DePuey EG, Gadiraju R, Clark J, Thompson L, Anstett F, Shwartz SC. Ordered subset expectation maximization and wide beam reconstruction "half-time" gated myocardial perfusion SPECT functional imaging: A comparison to "full-time" filtered backprojection. J Nucl Cardiol. 2008;14:547-63.

75. DePuey EG, Bommireddipalli S, Clark J, Thompson L, Srour Y. Wide beam reconstruction "quarter-time" gated myocardial perfusion SPECT functional imaging: A comparison to "fulltime" ordered subset expectation maximum. J Nucl Cardiol. 2009; 16:736-52.

76. Metz CE. The geometric transfer function component for scintillation camera collimators with straight parallel holes. Phys Med Biol. 1980;25:1059-70.

77. Tsui BM, Gullberg GT. The geometric transfer function for cone and fan beam collimators. Phys Med Biol. 1990;35:81-93.

78. Tsui BMW, Hu HB, Gillard DR, Gullberg GT. Implementation of simultaneous attenuation and detector response correction in SPECT. IEEE Trans Nucl Sci. 1988;35:778-83.

79. Tsui BM, Frey EC, Zhao X, Lalush DS, Johnston RE. McCartney WH. The importance and implementation of accurate three-dimensional compensation methods for quantitative SPECT. Phys Med Biol. 1994;39:509-30.

80. Tsui BMW, Zhao XD, Frey EC, Ju ZQ, Gullberg GT. Characteristics of reconstructed point response in three-dimensional spatially variant detector response compensation in SPECT. In: Grangeat P, Amans JL, editors. Three-Dimensional Image 
Reconstruction in Radiology and Nuclear Medicine. Computational Imaging and Vision. Dordrecht: Kluwer Academic Publishers; 1996. p. 509-30.

81. Philipe P, Bruyant J. Analytic and iterative reconstruction algorithms in SPECT. J Nucl Med. 2002;43:1343-58.

82. King MA, Tsui BM, Pan TS. Attenuation compensation for cardiac single-photon emission computed tomographic imaging: Part 1. Impact of attenuation and methods of estimating attenuation maps. J Nucl Cardiol. 1995;2:513-24.

83. European Council Directive 2013/59/Euratom on basic safety standards for protection against the dangers arising from exposure to ionising radiation. OJ of the EU 2014. L13; 57: 1-73.

84. Gambhir SS, Berman DS, Ziffer J, Nagler M, Sandler M, Patton $\mathrm{J}$, et al. A novel high-sensitivity rapid-acquisition single-photon cardiac imaging camera. J Nucl Med. 2009;50:635-43. https:// doi.org/10.2967/jnumed.108.060020.

85. Kennedy JA, Israel O, Frenkel A. 3D iteratively reconstructed spatial resolution map and sensitivity characterization of a dedicated cardiac SPECT camera. J Nucl Cardiol. 2014;21:443-52. https://doi.org/10.1007/s12350-013-9851-7.

86. Fiechter M, Gebhard C, Fuchs TA, Ghadri JR, Stehli J, Kazakauskaite E, et al. Cadmium-zinc-telluride myocardial perfusion imaging in obese patients. J Nucl Med. 2012;53:1401-6. https:// doi.org/10.2967/jnumed.111.102434.

87. Duvall WL, Slomka PJ, Gerlach JR, Sweeny JM, Baber U, Croft LB, et al. High-efficiency SPECT MPI: Comparison of automated quantification, visual interpretation, and coronary angiography. J Nucl Cardiol. 2013;20:763-73.

88. Hansen CL. The role of the translation table in cardiac image display. J Nucl Cardiol. 2006;13:571-5.

89. Mahmarian JJ, Boyce TM, Goldberg RK, Cocanougher MK, Roberts R, Verani MS. Quantitative exercise thallium-201 single photon emission computed tomography for the enhanced diagnosis of ischemic heart disease. J Am Coll Cardiol. 1990;15:31829.

90. Friedman J, Berman DS, Van Train K, Garcia EV, Bietendorf J, Prigent F, et al. Patient motion in thallium-201 myocardial SPECT imaging. An easily identified frequent source of artifactual defect. Clin Nucl Med. 1988;13:321-4.

91. Cooper JA, Neumann PH, McCandless BK. Effect of patient motion on tomographic myocardial perfusion imaging. J Nucl Med. 1992;33:1566-71.

92. Choi JY, Lee KH, Kim SJ, Kim SE, Kim BT, Lee SH, et al. Gating provides improved accuracy for differentiating artifacts from true lesions in equivocal fixed defects on technetium $99 \mathrm{~m}$ tetrofosmin perfusion SPECT. J Nucl Cardiol. 1998;5:395-401.

93. Ficaro EP, Fessler JA, Shreve PD, Kritzman JN, Rose PA, Corbett JR. Simultaneous transmission/emission myocardial perfusion tomography. Diagnostic accuracy of attenuation-corrected $99 \mathrm{mTc}$-sestamibi single-photon emission computed tomography. Circulation. 1996;93:463-73.

94. Fricke H, Fricke E, Weise R, Kammeier A, Lindner O, Burchert $\mathrm{W}$. A method to remove artifacts in attenuation-corrected myocardial perfusion SPECT. Introduced by misalignment between emission scan and CT-derived attenuation maps. J Nucl Med. 2004;45:1619-25.

95. Grossman GB, Garcia EV, Bateman T, Heller GV, Johnson LL, Folks RD, et al. Quantitative Tc-99m sestamibi attenuation-corrected SPECT: Development and multicenter trial validation of myocardial perfusion stress gender-independent normal database in obese population. J Nucl Cardiol. 2004;11:263-72.

96. Weiss AT, Berman DS, Lew AS, Nielsen J, Potkin B, Swan HJ, Waxman A, et al. Transient ischemic dilation of the left ventricle on stress thallium-201 scintigraphy: A marker of severe and extensive coronary artery disease. J Am Coll Cardiol. 1987;9:752-9.

97. McLaughlin MG, Danias PG. Transient ischemic dilation: A powerful diagnostic and prognostic finding of stress myocardial perfusion imaging. J Nucl Cardiol. 2002;9:663-7.

98. Xu Y, Arsanjani R, Clond M, Hyun M, Lemley M Jr, Fish M, et al. Transient ischemic dilation for coronary artery disease in quantitative analysis of same-day sestamibi myocardial perfusion SPECT. J Nucl Cardiol. 2012;19:465-73. https://doi.org/10. 1007/s12350-012-9527-8.

99. Hansen CL, Sangrigoli R, Nkadi E, Kramer M. Comparison of pulmonary uptake with transient cavity dilation after exercise thallium-201 perfusion imaging. J Am Coll Cardiol. 1999;33:1323-7.

100. Hansen CL, Cen P, Sanchez B, Robinson R. Comparison of pulmonary uptake with transient cavity dilation after dipyridamole Tl-201 perfusion imaging. J Nucl Cardiol. 2002;9:47-51.

101. Chouraqui P, Rodrigues EA, Berman DS, Maddahi J. Significance of dipyridamole-induced transient dilation of the left ventricle during thallium-201 scintigraphy in suspected coronary artery disease. Am J Cardiol. 1990;66:689-94.

102. Abidov A, Bax JJ, Hayes SW, Cohen I, Nishina H, Yoda S, et al. Integration of automatically measured transient ischemic dilation ratio into interpretation of adenosine stress myocardial perfusion SPECT for detection of severe and extensive CAD. J Nucl Med. 2004;45:1999-2007.

103. Gill JB, Ruddy TD, Newell JB, Finkelstein DM, Strauss HW, Boucher CA. Prognostic importance of thallium uptake by the lungs during exercise in coronary artery disease. N Engl J Med. 1987;317:1486-9.

104. Wackers FJT. On the bright side. J Nucl Cardiol. 2005;12:37880.

105. Williams KA, Schneider CM. Increased stress right ventricular activity on dual isotope perfusion SPECT: A sign of multivessel and/or left main coronary artery disease. J Am Coll Cardiol. 1999;34:420-7.

106. Williams KA, Hill KA, Sheridan CM. Noncardiac findings on dual-isotope myocardial perfusion SPECT. J Nucl Cardiol. 2003; 10:395-402.

107. Cerqueira MD, Weissman NJ, Dilsizian V, Jacobs AK, Kaul S, Laskey WK, et al. Standardized myocardial segmentation and nomenclature for tomographic imaging of the heart: A statement for healthcare professionals from the Cardiac Imaging Committee of the Council on Clinical Cardiology of the American Heart Association. J Nucl Cardiol. 2002;9:240-5.

108. Tilkemeier PL, Cooke CD, Grossman GB, McCallister BD, Ward RP. ASNC imaging guidelines for nuclear cardiology procedures: Standardized reporting of myocardial perfusion images. J Nucl Cardiol. 2009;16:165.

109. Xu Y, Hayes S, Ali I, Ruddy TD, Wells RG, Berman DS, et al. Automatic and visual reproducibility of perfusion and function measures for myocardial perfusion SPECT. J Nucl Cardiol. 2010;17:1050-7. https://doi.org/10.1007/s12350-010-9297-0.

110. Hachamovitch R, Berman DS, Kiat H, Cohen I, Cabico JA, Friedman J, et al. Exercise myocardial perfusion SPECT in patients without known coronary artery disease: Incremental prognostic value and use in risk stratification. Circulation. 1996;93:905-14.

111. Berman DS, Abidov A, Kang X, Hayes SW, Friedman JD, Sciammarella MG, et al. Prognostic validation of a 17-segment score derived from a 20-segment score for myocardial perfusion SPECT interpretation. J Nucl Cardiol. 2004;11:414-23.

112. Hachamovitch R, Berman DS, Shaw LJ, Kiat H, Cohen I, Cabico $\mathrm{JA}$, et al. Incremental prognostic value of myocardial perfusion 
single photon emission computed tomography for the prediction of cardiac death: Differential stratification for risk of cardiac death and myocardial infarction. Circulation. 1998;97:535-43.

113. Travin MI. The oft neglected rest study. J Nucl Cardiol. 2009;15:739-42.

114. Shaw LJ, Hendel RC, Heller GV, Borges-Neto S, Cerqueira M, Berman DS. Prognostic estimation of coronary artery disease risk with resting perfusion abnormalities and stress ischemia on myocardial perfusion SPECT. J Nucl Cardiol. 2009;15:762-73.

115. Hachamovitch R, Hayes SW, Friedman JD, Cohen I, Berman DS Comparison of the short-term survival benefit associated with revascularization compared with medical therapy in patients with no prior coronary artery disease undergoing stress myocardial perfusion single photon emission computed tomography. Circulation. 2003;107:2900-7.

116. Patel MR, Calhoon JH, Dehmer GJ, Grantham JA, Maddox TM, Maron DJ, et al. ACC/AATS/AHA/ASE/ASNC/SCAI/SCCT/ STS 2017 appropriate use criteria for coronary revascularization in patients with stable ischemic heart disease: A Report of the American College of Cardiology Appropriate Use Criteria Task Force, American Association for Thoracic Surgery, American Heart Association, American Society of Echocardiography, American Society of Nuclear Cardiology, Society for Cardiovascular Angiography and Interventions, Society of Cardiovascular Computed Tomography, and Society of Thoracic Surgeons. J Am Coll Cardiol. 2017;69:2212-41. https://doi.org/ 10.1016/j.jacc.2017.02.001.

117. Berman DS, Kang X, Van Train KF, Lewin HC, Cohen I, Areeda $\mathrm{J}$, et al. Comparative prognostic value of automatic quantitative analysis versus semiquantitative visual analysis of exercise myocardial perfusion single-photon emission computed tomography. J Am Coll Cardiol. 1998;32:1987-95.

118. Leslie WB, Tully SA, Yogendran MS, Ward LM, Nour KA, Metge CJ. Prognostic value of automated quantification of 99mTc-sestamibi myocardial perfusion imaging. J Nucl Med. 2005;46:204-11.

119. Rubeaux M, Xu Y, Germano G, Berman DS, Slomka PJ. Normal databases for the relative quantification of myocardial perfusion. Curr Cardiovasc Imaging Rep. 2016. https://doi.org/10.1007/ s12410-016-9385-x.

120. Berman DS, Kang X, Gransar H, Gerlach J, Friedman JD, Hayes $\mathrm{SW}$, et al. Quantitative assessment of myocardial perfusion abnormality on SPECT myocardial perfusion imaging is more reproducible than expert visual analysis. J Nucl Cardiol. 2009;16:45-53.

121. Iskandrian AS, Garcia EV, Faber T, Mahmarian JJ. Automated assessment of serial SPECT myocardial perfusion images. J Nucl Cardiol. 2009;16:6-9.

122. Mahmarian JJ, Cerqueira MD, Iskandrian AS. Regadenoson induces comparable left ventricular perfusion defects as adenosine: A quantitative analysis from the ADVANCE MPI 2 Trial. J Am Coll Cardiol Img. 2009;2:959-68.

123. Nakazato R, Berman DS, Gransar H, Hyun M, Miranda-Peats R, Kite FC, et al. Prognostic value of quantitative high-speed myocardial perfusion imaging. J Nucl Cardiol. 2012;19:1113-23. https://doi.org/10.1007/s12350-012-9619-5.

124. Slomka PJ, Nishina H, Berman DS, Kang X, Friedman JD, Hayes SW, Aladl UE, Germano G. Automatic quantification of myocardial perfusion stress-rest change: A new measure of ischemia. J Nucl Med. 2004;45:183-91.

125. Takeishi Y, Sukekawa H, Fujiwara S, Ikeno E, Sasaki Y, Tomoike H. Reverse redistribution of technetium-99m-sestamibi following direct PTCA in acute myocardial infarction. J Nucl Med. 1996;37:1289-94.
126. Weiss AT, Maddahi J, Lew AS, Shah PK, Ganz W, Swan HJ, et al. Reverse redistribution of thallium-201: A sign of nontransmural myocardial infarction with patency of the infarctrelated coronary artery. J Am Coll Cardiol. 1986;7:61-7.

127. Slomka PJ, Berman DS, Xu Y, Kavanagh P, Hayes SW, Dorbala $\mathrm{S}$, et al. Fully automated wall motion and thickening scoring system for myocardial perfusion SPECT: Method development and validation in large population. J Nucl Cardiol. 2012;19:291302. https://doi.org/10.1007/s12350-011-9502-9.

128. Sharir T, Kang X, Germano G, Bax JJ, Shaw LJ, Gransar H, et al. Prognostic value of post-stress left ventricular volume and ejection fraction by gated myocardial perfusion SPECT in women and men: Gender-related differences in normal limits and outcomes. J Nucl Cardiol. 2006;13:495-506.

129. Vallejo E, Dione DP, Bruni WL, Constable RT, Borek PP, Soares JP, et al. Reproducibility and accuracy of gated SPECT for determination of left ventricular volumes and ejection fraction: Experimental validation using MRI. J Nucl Med. 2000;41:87482.

130. Emmett L, Iwanochko RM, Freeman MR, Barolet A, Lee DS, Husain M. Reversible regional wall motion abnormalities on exercise technetium-99m-gated cardiac single photon emission computed tomography predict high-grade angiographic stenoses. J Am Coll Cardiol. 2002;39:991-8.

131. Sharir T, Bacher-Stier C, Dhar S, Lewin HC, Miranda R, Friedman JD, et al. Identification of severe and extensive coronary artery disease by postexercise regional wall motion abnormalities in Tc-99m sestamibi gated single-photon emission computed tomography. Am J Cardiol. 2000;86:1171-5.

132. Chen J, Garcia EV, Folks RD, Cooke CD, Faber TL, Tauxe L, et al. Onset of left ventricular mechanical contraction as determined by phase analysis of ECG-gated myocardial perfusion SPECT imaging: Development of a diagnostic tool for assessment of cardiac mechanical dyssynchrony. J Nucl Cardiol. 2005;12:687-95.

133. Henneman MM, Chen J, Ypenburg C, Dibbets P, Bleeker GB, Boersma E, et al. Phase analysis of gated myocardial perfusion single-photon emission computed tomography compared with tissue Doppler imaging for the assessment of left ventricular dyssynchrony. J Am Coll Cardiol. 2007;49:1708-14.

134. Henneman MM, Chen J, Dibbets-Schneider P, Stokkel MP, Bleeker GB, Ypenburg C, et al. Can LV dyssynchrony as assessed with phase analysis on gated myocardial perfusion SPECT predict response to CRT? J Nucl Med. 2007;48:1104-11.

135. Heydari B, Jerosch-Herold M, Kwong R. Imaging for planning of cardiac resynchronization therapy. J Am Coll Cardiovasc Imag. 2012;5:93-110

136. Tracy CM, Epstein AE, Darbar D, DiMarco JP, Dunbar SB, Estes NAM 3rd, et al. 2012 ACCF/AHA/HRS focused update of the 2008 guidelines for device-based therapy of cardiac rhythm abnormalities: a report of the American College of Cardiology Foundation/American Heart Association Task Force on Practice Guidelines. Circulation. 2012;126:00-1.

137. Duvernoy CS, Ficaro EP, Karabajakian MZ, Rose PA, Corbett JR. Improved detection of left main coronary artery disease with attenuation-corrected SPECT. J Nucl Cardiol. 2000;7:639-48.

138. Gibbons RJ, Balady GJ, Bricker JT, Chaitman BR, Fletcher GF, Froelicher VF, et al. ACC/AHA 2002 Guideline Update for Exercise Testing. Circulation. 2002;106:1883-92. https://doi.org/ 10.1161/01.CIR.0000034670.06526.15

139. Sciagrà R, Leoncini M, Marcucci G, Dabizzi RP, Pupi A. Technetium-99m sestamibi imaging to predict left ventricular ejection fraction outcome after revascularisation in patients with chronic coronary artery disease and left ventricular dysfunction: 
comparison between baseline and nitrate-enhanced imaging. Eur J Nucl Med. 2001;28:680-7.

140. Leoncini M, Sciagrà R, Bellandi F, Maioli M, Sestini S, Marcucci G, et al. Low-dose dobutamine nitrate-enhanced technetium 99m sestamibi gated SPECT versus low-dose dobutamine echocardiography for detecting reversible dysfunction in ischemic cardiomyopathy. J Nucl Cardiol. 2002;9:402-6.

141. Dilsizian V, Bacharach SL, Beanlands RS, Bergmann SR, Delbeke D, Dorbala $\mathrm{S}$, et al. ASNC imaging guidelines/SNMMI procedure standard for positron emission tomography (PET) nuclear cardiology procedures. J Nucl Cardiol. 2016;23:1187226.

142. Schinkel AF, Bax JJ, Poldermans D, Elhendy A, Ferrari R, Rahimtoola SH. Hibernating myocardium: Diagnosis and patient outcomes. Curr Probl Cardiol. 2007;32:375-410.

143. Dilsizian V, Rocco TP, Freedman NM, Leon MB, Bonow RO. Enhanced detection of ischemic but viable myocardium by the reinjection of thallium after stress-redistribution imaging. $\mathrm{N}$ Engl J Med. 1990;323:141-6.

144. He ZX, Medrano R, Hays JT, Mahmarian JJ, Verani MS. Nitroglycerin-augmented 201T1 reinjection enhances detection of reversible myocardial hypoperfusion. A randomized, doubleblind, parallel, placebo-controlled trial. Circulation. 1997;95:1799-805.

145. Dilsizian V, Bonow RO. Current diagnostic techniques of assessing myocardial viability in patients with hibernating and stunned myocardium. Circulation. 1993;87:1-20.

146. Uebleis C, Hellweger S, Laubender RP, Becker A, Sohn HY, Lehner S, et al. The amount of dysfunctional but viable myocardium predicts long-term survival in patients with ischemic cardiomyopathy and left ventricular dysfunction. Int $\mathbf{J}$ Cardiovasc Imaging. 2013;29:1645-53. https://doi.org/10.1007/s10554013-0254-2.

147. Ling LF, Marwick TH, Flores DR, Jaber WA, Brunken RC, Cerqueira MD, et al. Identification of therapeutic benefit from revascularization in patients with left ventricular systolic dysfunction: Inducible ischemia versus hibernating myocardium. Circ Cardiovasc Imaging. 2013;6:363-72. https://doi.org/10. 1161/CIRCIMAGING.112.000138.

148. Bax JJ, Delgado V. Myocardial viability as integral part of the diagnostic and therapeutic approach to ischemic heart failure. $\mathrm{J}$ Nucl Cardiol. 2015;22:229-45. https://doi.org/10.1007/s12350015-0096-5.

149. Beanlands RS, Nichol G, Huszti E, Humen D, Racine N, Freeman $\mathrm{M}$, et al. F-18-fluorodeoxyglucose positron emission tomography imaging-assisted management of patients with severe left ventricular dysfunction and suspected coronary disease: a randomized, controlled trial (PARR-2). J Am Coll Cardiol. 2007;50:2002-12.

150. Cleland JG, Calvert M, Freemantle N, Arrow Y, Ball SG, Bonser RS, et al. The heart failure revascularisation trial (HEART). Eur J Heart Fail. 2011;13:227-33. https://doi.org/10.1093/eurjhf/ hfq230.

151. Velazquez EJ, Lee KL, Deja MA, Jain A, Sopko G, Marchenko A, et al. Coronary-artery bypass surgery in patients with left ventricular dysfunction. N Engl J Med. 2011;364:1607-16. https://doi.org/10.1056/NEJMoa1100356.

152. Velazquez EJ, Lee KL, Jones RH, Al-Khalidi HR, Hill JA, Panza $\mathrm{JA}$, et al. Coronary-artery bypass surgery in patients with ischemic cardiomyopathy. N Engl J Med. 2016;374:1511-20. https://doi.org/10.1056/NEJMoa1602001.

153. Bonow RO, Maurer G, Lee KL, Holly TA, Binkley PF, Desvigne-Nickens $P$, et al. Myocardial viability and survival in ischemic left ventricular dysfunction. $N$ Engl $J$ Med. 2011;364:1617-25. https://doi.org/10.1056/NEJMoa1100358.

154. Yancy CW, Jessup M, Bozkurt B, Butler J, Casey DE Jr, Drazner $\mathrm{MH}$, et al. 2013 ACCF/AHA guideline for the management of heart failure: A report of the American College of Cardiology Foundation/American Heart Association Task Force on practice guidelines. Circulation. 2013;128:e240-327. https://doi.org/10. 1161/CIR.0b013e31829e8776.

155. Wells RG, Timmins R, Klein R, Lockwood J, Marvin B, deKemp RA, et al. Dynamic SPECT measurement of absolute myocardial blood flow in a porcine model. J Nucl Med. 2014;55:1685-91.

156. Shrestha U, Sciammarella M, Alhassen F, Yeghiazarians Y, Ellin $\mathrm{J}$, Verdin E, et al. Measurement of absolute myocardial blood flow in humans using dynamic cardiac SPECT and 99mTctetrofosmin: Method and validation. J Nucl Cardiol. 2017;24:268-77.

157. Hsu B, Hu LH, Yang BH, Chen LC, Chen YK, Ting CH, et al. SPECT myocardial blood flow quantitation toward clinical use: a comparative study with 13N-Ammonia PET myocardial blood flow quantitation. Eur J Nucl Med Mol Imaging. 2017;44:11728.

158. Shiraishi S, Sakamoto F, Tsuda N, Yoshida M, Tomiguchi S, Utsunomiya D, Ogawa $\mathrm{H}$, et al. Prediction of left main or 3vessel disease using myocardial perfusion reserve on dynamic thallium-201 single-photon emission computed tomography with a semiconductor gamma camera. Circ J. 2015;79:623-31. https:// doi.org/10.1253/circj.CJ-14-0932.

159. Ben Bouallègue F, Roubille F, Lattuca B, Cung TT, Macia JC, Gervasoni $\mathrm{R}$, et al. SPECT myocardial perfusion reserve in patients with multivessel coronary disease: correlation with angiographic findings and invasive fractional flow reserve measurements. J Nucl Med. 2015;56:1712-7.

160. Murthy VL, Naya M, Foster CR, Hainer J, Gaber M, Di Carli G, et al. Improved cardiac risk assessment with noninvasive measures of coronary flow reserve. Circulation. 2011;124:2215-24.

161. Herzog BA, Husmann L, Gaemperli O, Valenta I, Siegrist PT, Tay FM, et al. Long-term prognostic value of $13 \mathrm{~N}$-ammonia myocardial perfusion positron emission tomography added value of coronary flow reserve. J Am Coll Card. 2009;54:150-6.

162. Ziadi MC, Dekemp RA, Williams KA, Guo A, Chow BJ, Renaud $\mathrm{JM}$, et al. Impaired myocardial flow reserve on rubidium- 82 positron emission tomography imaging predicts adverse outcomes in patients assessed for myocardial ischemia. J Am Coll Cardiol. 2011;58:740-8.

163. Gould KL, Johnson NP, Bateman TM, Beanlands RS, Bengel FM, Bober R, et al. Anatomic versus physiologic assessment of coronary artery disease. Role of coronary flow reserve, fractional flow reserve, and positron emission tomography imaging in revascularization decision-making. $\mathrm{J}$ Am Coll Cardiol. 2013;62:1639-53.

164. Askew JW, Miller TD, Ruter RL, Jordan LG, Hodge DO, Gibbons RJ, et al. Early image acquisition using a solid-state cardiac camera for fast myocardial perfusion imaging. J Nucl Cardiol. 2011;18:840-6.

165. Duvall WL, Wijetunga MN, Klein TM, Razzouk L, Godbold J, Croft LB, et al. The prognosis of a normal stress-only Tc-99m myocardial perfusion imaging study. J Nucl Cardiol. 2010;17:370-7. https://doi.org/10.1007/s12350-010-9210-x.

166. Einstein AJ, Blankstein R, Andrews H, Fish M, Padgett R, Hayes $\mathrm{SW}$, et al. Comparison of image quality, myocardial perfusion, and left ventricular function between standard imaging and single-injection ultra-low-dose imaging using a high-efficiency SPECT camera: The MILLISIEVERT study. J Nucl Med. 2014;55:1430-7. 
167. Thompson RC, O'Keefe JH, McGhie AI, Bybee KA, Thompson EC, Bateman TM. Reduction of SPECT MPI radiation dose using contemporary protocols and technology. JACC Cardiovasc Imaging. 2017. https://doi.org/10.1016/j.jcmg.2017.03.008

168. Duvall WL, Guma KA, Kamen J, Croft LB, Parides M, George $\mathrm{T}$, et al. Reduction in occupational and patient radiation exposure from myocardial perfusion imaging: impact of stress-only imaging and high-efficiency SPECT camera technology. J Nucl Med. 2013;54:1251-7. https://doi.org/10.2967/jnumed.112. 112680.

169. Nabi F, Kassi M, Muhyieddeen K, Chang SM, Xu J, Peterson LE, et al. Optimizing evaluation of patients with low-to-intermediaterisk acute chest pain: A randomized study comparing stress myocardial perfusion tomography incorporating stress-only imaging versus cardiac CT. J Nucl Med. 2016;57:378-84. https:// doi.org/10.2967/jnumed.115.166595.

170. Thompson RC, Allam AH. More risk factors, less ischemia, and the relevance of MPI testing. J Nucl Cardiol. 2015;22:552-4.

171. Mahmarian JJ. Implementation of stress-only imaging: What will it take? J Nucl Cardiol. 2017;24:821-5. https://doi.org/10.1007/ s12350-015-0346-6.

172. Cerqueira MD, Allman KC, Ficaro EP, Hansen CL, Nichols KJ, Thompson $\mathrm{RC}$, et al. Recommendations for reducing radiation exposure in myocardial perfusion imaging. J Nucl Cardiol. 2010;17:709-18. https://doi.org/10.1007/s12350-010-9244-0.

173. Heller GV, Bateman TM, Johnson LL, Cullom SJ, Case JA, Galt JR, et al. Clinical value of attenuation correction in stress-only Tc-99m sestamibi SPECT imaging. J Nucl Cardiol. 2004;11:273-81.

174. Thomas GS, Cullom SJ, Kitt TM, Feaheny KM, Ananthasubramaniam K, Gropler RJ, et al. The EXERRT trial: EXErcise to Regadenoson in Recovery Trial: A phase 3b, open-label, parallel group, randomized, multicenter study to assess regadenoson administration following an inadequate exercise stress test as compared to regadenoson without exercise for myocardial perfusion imaging using a SPECT protocol. J Nucl Cardiol. 2017;24:788-802. https://doi.org/10.1007/s12350-017-0813-3.

175. Mahmarian JJ. Regadenoson stress during low-level exercise: The EXERRT trial-does it move the needle? J Nucl Cardiol. 2017;24:803-8. https://doi.org/10.1007/s12350-017-0873-4.

176. Duvall WL, Baber U, Levine EJ, Croft LB, Henzlova MJ. A model for the prediction of a successful stress-first Tc-99m SPECT MPI. J Nucl Cardiol. 2012;19:1124-34.

177. Gowdar S, Chaudhry W, Ahlberg AW, Henzlova MJ, Duvall WL. Triage of patients for attenuation-corrected stress-first Tc99m SPECT MPI using a simplified clinical pre-test scoring model. J Nucl Cardiol. 2017. https://doi.org/10.1007/s12350017-0832-0.

178. Gemignani AS, Muhlebach SG, Abbott BG, Roye GD, Harrington DT, Arrighi JA. Stress-only or stress/rest myocardial perfusion imaging in patients undergoing evaluation for bariatric surgery. J Nucl Cardiol. 2011;18:886-92. https://doi.org/10. 1007/s12350-011-9405-9.

179. Mathur S, Heller GV, Bateman TM, Ruffin R, Yekta A, Katten $\mathrm{D}$, et al. Clinical value of stress-only Tc-99m SPECT imaging: Importance of attenuation correction. $\mathrm{J}$ Nucl Cardiol. 2013;20:27-37. https://doi.org/10.1007/s12350-012-9633-7.

180. Bloom SA, Meyers K. Reducing radiation to patients and improving image quality in a real-world nuclear cardiology laboratory. J Nucl Cardiol. 2017;24:1871-7. https://doi.org/10. 1007/s12350-017-0851-x.

181. DePuey EG, Mahmarian JJ, Miller TD, Einstein AJ, Hansen CL, Holly TL, et al. ASNC preferred practice statement: Patientcentered imaging. J Nucl Cardiol. 2012;19:185-215.
182. Einstein AJ, Berman DS, Min JK, Hendel RC, Gerber TC, Carr JJ, et al. Patient centered imaging: Shared decision making for cardiac imaging procedures with exposure to ionizing radiation. $\mathbf{J}$ Am Coll Cardiol. 2014;63:1480-9.

183. Jha S, Boonn W. Patient-centered imaging: Opportunities and challenges. J Am Coll Rad. 2012;9:157-9.

184. Swensen SJ. Patient-centered imaging. Am J Med. 2012;125:115-7. https://doi.org/10.1016/j.amjmed.2011.06.002.

185. Thompson RC, Burgett EV. Patient centered imaging and the dose of radiopharmaceuticals. J Nucl Cardiol. 2016;23:143-4. https://doi.org/10.1007/s12350-015-0263-8.

186. van Dijk JD, Jager PL, Ottervanger JP, de Boer J, Oostdijk AHJ, Engbers EM, et al. Development and validation of a patienttailored dose regime in myocardial perfusion imaging using conventional SPECT. J Nucl Cardiol. 2016;23:134-42. https:// doi.org/10.1007/s12350-015-0246-9.

187. van Dijk JD, Jager PL, Mouden M, Slump CH, Ottervanger JP, de Boer J, et al. Development and validation of a patient-tailored dose regime in myocardial perfusion imaging using CZTSPECT. J Nucl Cardiol. 2014;21:1158-67. https://doi.org/10. 1007/s12350-014-9942-0.

188. Herzog BA, Buechel RR, Katz R, Brueckner M, Husmann L, Burger IA, et al. Nuclear myocardial perfusion imaging with a cadmium-zinc-telluride detector technique: Optimized protocol for scan time reduction. J Nucl Med. 2010;51:46-51. https://doi. org/10.2967/jnumed.109.065532.

189. Greenland P, Alpert JS, Beller GA, Benjamin EJ, Budoff MJ, Fayad ZA, et al. 2010 ACCF/AHA guideline for assessment of cardiovascular risk in asymptomatic adults: A report of the American College of Cardiology Foundation/American Heart Association Task Force on Practice Guidelines. J Am Coll Cardiol. 2010;56:e50-103. https://doi.org/10.1016/j.jacc.2010.09. 001.

190. Thompson RC, McGhie AI, Moser KW, O'Keefe JH Jr, Stevens TL, House J, et al. Clinical utility of coronary artery calcium scoring after nonischemic myocardial perfusion imaging. J Nucl Cardiol. 2005;12:392-400.

191. Rozanski A, Gransar H, Shaw LJ, Kim J, Miranda-Peats L, Wong $\mathrm{ND}$, et al. Impact of coronary artery calcium scanning on coronary risk factors and downstream testing the EISNER (Early Identification of Subclinical Atherosclerosis by Noninvasive Imaging Research) prospective randomized trial. J Am Coll Cardiol. 2011;57:1622-32. https://doi.org/10.1016/j.jacc.2011. 01.019 .

192. Schenker MP, Dorbala S, Hong EC, Rybicki FJ, Hachamovitch $\mathrm{R}$, Kwong RY, et al. Interrelation of coronary calcification, myocardial ischemia, and outcomes in patients with intermediate likelihood of coronary artery disease: A combined positron emission tomography/computed tomography study. Circulation. 2008;117:1693-700.

https://doi.org/10.1161/ CIRCULATIONAHA.107.717512.

193. Chang SM, Nabi F, Xu J, Pratt CM, Mahmarian AC, Frias ME, et al. Value of CACS compared with ETT and myocardial perfusion imaging for predicting long-term cardiac outcome in asymptomatic and symptomatic patients at low risk for coronary disease: Clinical implications in a multimodality imaging world. JACC Cardiovasc Imaging. 2015;8:134-44. https://doi.org/10. 1016/j.jcmg.2014.11.008.

194. Engbers EM, Timmer JR, Ottervanger JP, Mouden M, Knollema $\mathrm{S}$, Jager PL. Prognostic value of coronary artery calcium scoring in addition to single-photon emission computed tomographic myocardial perfusion imaging in symptomatic patients. Circ Cardiovasc Imaging. 2016. https://doi.org/10.1161/circimaging. 115.003966. 
195. Einstein AJ, Johnson LL, Bokhari S, Son J, Thompson RC, Bateman TM, et al. Agreement of visual estimation of coronary artery calcium from low-dose CT attenuation correction scans in hybrid PET/CT and SPECT/CT with standard Agatston score. J Am Coll Cardiol. 2010;56:1914-21. https://doi.org/10.1016/j. jacc.2010.05.057.

196. Takx RA, Išgum I, Willemink MJ, van der Graaf Y, de Koning $\mathrm{HJ}$, Vliegenthart R, et al. Quantification of coronary artery calcium in nongated $\mathrm{CT}$ to predict cardiovascular events in male lung cancer screening participants: results of the NELSON study. J Cardiovasc Comput Tomogr. 2015;9:50-7. https://doi.org/10. 1016/j.jcct.2014.11.006.

197. Mylonas I, Kazmi M, Fuller L, deKemp RA, Yam Y, Chen L, et al. Measuring coronary artery calcification using positron emission tomography-computed tomography attenuation correction images. Eur Heart J Cardiovasc Imaging. 2012;13:786-92. https://doi.org/10.1093/ehjci/jes079. 